\title{
GABA facilitates spike propagation at branch points of sensory axons in the spinal cord
}

Krishnapriya Hari ${ }^{1,6}$, Ana M. Lucas-Osma ${ }^{1,2,6}$, Krista Metz ${ }^{1}$, Shihao Lin ${ }^{1}$, Noah Pardell ${ }^{1}$, David A. Roszko $^{1}$, Sophie Black ${ }^{1}$, Anna Minarik ${ }^{1}$, Rahul Singla ${ }^{1}$, Marilee J. Stephens ${ }^{1,3}$, Karim Fouad ${ }^{1,2}$, Kelvin E. Jones ${ }^{1,4}$, Monica A. Gorassini ${ }^{1,3}$, Keith K. Fenrich ${ }^{1,2,7}$, Yaqing Li ${ }^{1,5,7}$ and David J. Bennett ${ }^{1,2,7,8^{*}}$

${ }^{1}$ Neuroscience and Mental Health Institute, University of Alberta, Edmonton, AB, T6G 2R3, Canada

${ }^{2}$ Faculty of Rehabilitation Medicine, University of Alberta, Edmonton, AB, T6G 2G4, Canada

${ }^{3}$ Department of Biomedical Engineering, Faculty of Medicine and Dentistry, T6G 2V2, University of Alberta, Edmonton, AB, Canada. ${ }^{4}$ Faculty of Kinesiology, Sport and Recreation, University of Alberta, Edmonton, AB, T6G 2H9, Canada. ${ }^{5}$ Present address: Department of Physiology, Emory University, Atlanta, GA, 30322, USA. ${ }^{6}$ These authors contributed equally. ${ }^{7}$ Senior author. ${ }^{8}$ Lead Contact.

*Corresponding author: bennettd@ualberta.ca. 


\begin{abstract}
Movement and posture depend on sensory feedback that is regulated by specialized GABAergic neurons $\left(\mathrm{GAD}^{+}\right)$that form axo-axonic contacts onto myelinated proprioceptive sensory axons and are thought to be inhibitory. However, we report here that activating GAD2 ${ }^{+}$neurons, directly with optogenetics or indirectly by cutaneous stimulation, facilitates sensory feedback to motoneurons in awake rodents and humans. GABAA receptors and GAD2 ${ }^{+}$contacts adjacent to nodes of Ranvier at branch points of sensory axons cause this facilitation, preventing spike propagation failure that is otherwise common without GABA. GABAA receptors are generally lacking from axon terminals (unlike $\mathrm{GABA}_{\mathrm{B}}$ ) and do not inhibit transmitter release onto motoneurons, disproving the long-standing assumption that $\mathrm{GABA}_{\mathrm{A}}$ receptors cause presynaptic inhibition. GABAergic innervation of nodes near branch points allows individual branches to function autonomously, with GAD2 ${ }^{+}$neurons regulating which branches conduct, adding a computational layer to the neuronal networks generating movement and likely generalizing to other CNS axons.
\end{abstract}

\title{
Main
}

The ease with which animals move defies the complexity of the underlying neuronal circuits, which include corticospinal tracts (CSTs) that coordinate skilled movement, spinal interneurons that form central patterns generators (CGPs) for walking, and motoneurons that ultimately drive the muscles ${ }^{1}$. Sensory feedback ensures the final precision of such motor acts, with proprioceptive feedback to motoneurons producing a major part of the muscle activity in routine movement and posture ${ }^{2-4}$, without which severe ataxia occurs ${ }^{5}$. Proprioceptive sensory feedback is regulated by specialized GABAergic neurons (GAD2 $2^{+}$; abbreviated GABA axo neurons) that form axo-axonic connections onto the sensory axon terminals ${ }^{6-8}$. These neurons are thought to produce presynaptic inhibition of sensory feedback to motoneurons ${ }^{9-11}$ and possibly limit inappropriate sensory feedback ${ }^{3,4,7}$. However, during movement the CST, CPG and even sensory neurons all augment GABAaxo neuron activity ${ }^{11-16}$ right at a time when sensory feedback is known to be increased to ensure precision and postural stability ${ }^{2-4}$, raising the question of whether GABA axo neurons have a yet undescribed excitatory action.

The long-standing view that GABAergic neurons produce presynaptic inhibition of proprioceptive sensory axon terminals in adult mammals actually lacks direct evidence, largely because of the difficulty in recording from these small terminals and the technical limitations of previously employed 
reduced spinal cord preparations (immature) $)^{7,17}$ or anesthetized animals, since anesthetics themselves modulate GABA receptors ${ }^{9,18}$. Thus, in this paper we used optogenetic approaches to directly target GABA $_{a x o}$ neurons in awake animals and in isolated whole adult spinal cord preparations. Surprisingly, we found that optogenetically activating these GABAergic neurons markedly facilitates sensory axon transmission to motoneurons, throwing into doubt presynaptic inhibition.

GABAA receptors on sensory axon terminals have long been assumed to cause presynaptic inhibition, based on indirect evidence ${ }^{11,18}$, though their mode of action is rather counterintuitive. Sensory axons, like many other axons, have high intracellular chloride concentrations, leading to an outward chloride ion flow through activated $\mathrm{GABA}_{\mathrm{A}}$ receptors ${ }^{11,19,20}$. Thus, GABAA receptors cause a depolarization of sensory axons (primary afferent depolarization, PAD) ${ }^{11,16,21-23}$. PAD and associated GABAA receptors have variously been theorized to cause presynaptic inhibition by depolarization-dependent inactivation or shunting of sodium currents at the sensory axon terminals ${ }^{22,24}$. However, we do not even know if terminals of large myelinated proprioceptive sensory axons express GABAA receptors at all, despite their demonstrated innervation by GABA axo neurons ${ }^{6}$. These terminals appear to lack the $\alpha 5$ subunit of extrasynaptic GABAA $_{A}$ receptors ${ }^{16}$, but this leaves open the possibility that they express synaptic $\mathrm{GABA}_{\mathrm{A}}$ or $\mathrm{GABA}_{\mathrm{B}}$ receptors. We thus examined this question here and unexpectedly found that synaptic $\mathrm{GABA}_{\mathrm{A}}$ receptors are generally not at these axon terminals, but are instead near sodium channels (Nav) at the nodes of Ranvier throughout the myelinated regions of the axon, together with innervation by GABAaxo neurons. What then is the function of such nodal GABAA receptors?

An unexplored possibility is that the depolarizing action of nodal GABAA receptors (and GABAaxo neurons) aids sodium spike propagation between axon nodes. This has not previously been considered, as spikes are thought to securely propagate from node to node, at least in the orthodromic direction ${ }^{16}$. Myelinated proprioceptive axons branch extensively in the spinal $\operatorname{cord}^{16}$ (Fig. 1a) and each branch point poses a theoretical risk for spike propagation failure ${ }^{25,26}$. However, branch points are always located near nodes $(\mathrm{Nav})^{16}$, likely to minimize this failure. Nevertheless, indirect evidence has suggested that propagation failure can occur ${ }^{27-30}$. Thus, in the present study we sought direct evidence of nodal spike failure at branch points and examined whether nodal GABA and PAD facilitates afferent conduction by preventing this failure. We already know that PAD lowers the threshold for initiating axon spikes by extracellular stimulation $^{31}$, and even initiates spikes ${ }^{16}$, but do not know whether it aids normal spike propagation. We found that spike propagation depends so heavily on nodal GABA that blocking GABA action makes the majority of proprioceptive sensory axons fail to propagate spikes to motoneurons, and 
thus GABA provides a powerful mechanism to turn on specific nodes and branches to regulate sensory feedback.

\section{Results}

\section{Nodal $\mathrm{GABA}_{A}$ and terminal $\mathrm{GABA}_{B}$ receptors.}

To visualize how GABA regulates axons we examined the distribution of GABAA receptor subunit immunolabelling on nodes and terminals in large myelinated proprioceptive sensory axons, including $\alpha 5$ subunits that are extrasynaptic, $\alpha 1$ and $\alpha 2$ subunits that are mostly synaptic, and ubiquitous $\gamma 2$ subunits $^{32}$. We labelled sensory axons with neurobiotin injections in the rat spinal cord (Fig. 1) or fluorescent reporters in VGLUT1 ${ }^{\mathrm{Cre} /+}$ mice (Extended Data Fig. 1), and reconstructed them in 3D. $\mathrm{GABA}_{\mathrm{A}}$ receptors containing $\alpha 5, \alpha 1, \alpha 2$ and $\gamma 2$ subunits were expressed on these axons, especially near sodium channels (<6 $\mu$ m away; Fig. 1d-h, Extended Data Fig. 1). Specifically, GABAA receptors were on large myelinated $1^{\text {st }}$ and $2^{\text {nd }}$ order branches in the dorsal and ventral horn (Figs. 1d-e,h; Extended Data Fig. 1) near their nodes (identified by large sodium channel clusters, paranodal Caspr, and an axonal taper; $<10 \mu \mathrm{m}$ away; Figs. 1c,h,j), and on short unmyelinated terminal branches in the dorsal horn $\left(3^{\text {rd }}\right.$ order; Figs. $\left.1 \mathrm{~b}, \mathrm{~g}\right)$, the latter near nodes on $2^{\text {nd }}$ order branches $(<100 \mu \mathrm{m}$ away). In contrast, GABAA receptors were mostly absent from the long unmyelinated terminal branches contacting motoneurons in the ventral horn ( $3^{\text {rd }}$ order; Figs. 1b,f,g; Extended Data Fig. 1a-e), which also generally lacked sodium channels ${ }^{16}$. This left GABAA receptors on average far from the terminal boutons contacting motoneurons $\left(\sim 500 \mu \mathrm{m}\right.$; Fig. 1h) relative to the axon space constant $\left(\lambda_{\mathrm{s}} \sim 90 \mu \mathrm{m}\right)$, the majority in dorsal and intermediate laminae. Nodes were widely spaced, as were branch points $(\sim 50 \mu \mathrm{m}$ separation, Fig. 1i), but branch points were always near nodes (100\%, within $7 \mu \mathrm{m}$; Nav; Fig. 1c-e,i) and associated GABAA receptors. While nodes sometimes occurred without branching (49\%), the majority of nodes expressing $\mathrm{GABA}_{\mathrm{A}}$ receptors were at branch points (Fig. 1j), implying an importance for branch point GABA. In contrast, $\mathrm{GABA}_{\mathrm{B}}$ receptors were found mostly on terminal branches in the ventral horn where boutons had dense receptor expression (Figs. 1f-h), and not usually on larger myelinated ventral or dorsal branches (not at nodes; Figs. 1e,g,j; Extended Data Fig. 1f).

\section{Propagation failure in dorsal horn axon branches.}

Considering that GABAA receptors are expressed in large myelinated branches of proprioceptive axons, we next directly recorded from of these branches in the dorsal horn of rat and mouse spinal cords (Figs. 
2 and 3) to examine whether spike propagation depends on these receptors. When we stimulated the dorsal root (DR) containing the axon branch, an all-or-nothing spike was recorded in many branches (Figs. 2b, 3d) at the latency of the fastest afferent volley that arrived at the spinal cord (group I afferents; EC in Fig. 2b). However, in other axon branches this spike did not occur ( 20\%), but at the same latency there was a small all-or-nothing residual spike (failure potential, FP). This FP was indicative of a spike activating a distant node, but failing to propagate further to the recording site, leaving only its passively attenuated potential, with smaller FPs reflecting more distal failure points in the spinal cord (Figs. 2c-g, 3e-f). Failure never occurred in the DR itself (Fig. 2f). The failing branches with FPs were otherwise indistinguishable from non-failing axon branches, exhibiting full spikes $(>60$ $\mathrm{mV}$ ) with current injection pulses (Fig. 2cii, g), and low conductances and resting potentials $(\sim-65 \mathrm{mV}$, Fig. 2h), ruling out penetration injury. With high repetitive DR stimulation rates all branches (100\%) exhibited propagation failure and an associated FP (Fig. 2e-g), again with the FP implying that the spike is reliably initiated in the DR, but incompletely propagates within the spinal cord.

Axon spike failure was voltage dependent: in branches with failing spikes (FPs) depolarizations that brought the axon closer to threshold enabled full DR-evoked spikes (via current injection, Fig. 2ci; or spontaneous depolarization Fig. 2d). Also, in branches without spike failure at rest (secure spikes) a steady hyperpolarizing current induced spike failure (FP), with more branches failing with increasing hyperpolarization (Extended Data Fig. 2). With increasing hyperpolarization, nodes failed progressively more distal to the electrode, causing abrupt drops in the overall spike amplitude with each failure and a characteristic delay in the nodal spike prior to failure (Extended Data Fig. 2a-d). Simulating spike propagation by applying a brief current pulse to mimic the current arriving from an upstream node (and FP) yielded similar results, with full spikes evoked at rest, but hyperpolarization leading to a spike delay and then failure (Extended Data Fig. 3). Large depolarizations inactivated spikes, though outside of the physiological range ( $>-50 \mathrm{mV}$, Extended Data Fig. 3b-c).

\section{Nodal spike facilitation by GABA.}

Since sensory axons are tonically depolarized by spontaneous GABA activity ${ }^{16}$, we wondered whether this GABA aids propagation. Blocking extrasynaptic $\alpha 5$ GABAA receptors (with L655708) or all GABAA receptors (with gabazine) increased the incidence of spike failure (to $\sim 45 \%$ and $65 \%$, respectively; Fig. 2f) and sensitivity to hyperpolarization (Extended Data Fig. 2e-h), without altering overall spike properties (Fig. $2 \mathrm{~g}$ ), implying that spike propagation is highly dependent on nodal GABA 
receptors. Application of 5-HT to mimic natural brainstem-derived 5-HT also increased failure (Fig. 2f), likely via its indirect inhibition of GABAA receptor activity ${ }^{33}$.

\section{Nodal spike facilitation by $\mathrm{GABA}_{\text {axo }}$ neuron activation.}

To examine whether $\mathrm{GABA}_{\mathrm{axo}}$ neurons facilitate spike propagation, we expressed light-sensitive channelrhodopsin-2 (ChR2) in GAD2 ${ }^{+}$neurons in adult GAD2 ${ }^{\text {CreER/+ }} ; \mathrm{R}_{2} 6^{\text {LSL-ChR2-EYFP }}$ mice (termed GAD2//ChR2-EYFP mice, Fig. 3). A brief light pulse (5 - $10 \mathrm{~ms}$ ) produced a long-lasting depolarization and spiking in these GABAaxo neurons (Fig. 3a), followed by a longer lasting GABAAmediated depolarization (PAD) of proprioceptive axons at a monosynaptic latency that was blocked by gabazine (Fig. 3a-b). In these mice, spikes in proprioceptive axons failed with a similar incidence as observed in rats (Figs. 3c-h), but the light-evoked PAD prevented this failure (Fig. 3e-g), similar to direct depolarization. In branches with secure non-failing spikes, light had minor effects (Fig. 3d), but blocking GABAA receptors again increased the incidence of spike failure (Fig. 3h).

In GAD2//ChR2-EYFP or GAD2//ChR2-EYFP//tdTom mice the EYFP and tdTom reporters labelled GABAergic neurons (Fig. 3k; $\mathrm{VGAT}^{+}, \mathrm{GAD}^{+}$and $\mathrm{VGLUT1}^{-}$) residing near the central canal and throughout much of the dorsal horn (Fig. 3i-o). These neurons densely innervated the dorsal horn with terminal boutons (Fig. 3j,1,n), and less densely innervated both the ventral horn and dorsal columns (Fig. 31,m,o), allowing GABAergic innervation of sensory axons along their entire length. They made both synaptic $\left(\mathrm{VGAT}^{+}\right)$and perisynaptic contacts all along proprioceptive sensory axons, both at nodes and sensory axon terminals on motoneurons (Figs. 3k, and 1e), confirming their identity as GABAaxo neurons.

\section{Computer simulations of branch point failure and rescue by GABA.}

To establish that spike failure arises at the branch points where GABA can influence them, we generated a computer simulation of a proprioceptive sensory axon arbour in the spinal cord ${ }^{34}$. With simulated DR stimulation, spike failure occurred distal to complex branch points (at nodes N2 and N3 in Extended Data Fig. 4a-b) that had associated increases in net conductance, which shunted the nodal currents. Simulated nodal GABAA receptor activation rescued these failed spikes, with increasing GABAA activation ( $\mathrm{g}_{\mathrm{GABA}}$ ) preventing more branch point failures (Extended Data Fig. 4c). In contrast, when we moved all these GABAA receptors to the terminals, then their activation did not rescue failed spikes (Extended Data 4d). This is because GABAA-induced depolarizations (PAD) were attenuated 
sharply with distance $\left(\lambda_{\mathrm{s}} \sim 90 \mu \mathrm{m}\right)$; so only nodal, and not terminal, induced PAD was visible at the dorsal columns (Extended Data Fig. 4a,g-h), in agreement with previous terminal recordings ${ }^{16}$.

\section{Spike facilitation by sensory evoked GABA axo activity}

We next examined whether natural activation of $\mathrm{GABA}_{\text {axo }}$ neurons affects proprioceptive axon conduction. GABA axo neurons are indirectly activated by sensory activity via two variants of a trisynaptic circuit, where sensory axons drive excitatory neurons that activate GABAaxo neurons and cause PAD: one driven by cutaneous afferents and the other by proprioceptive afferents (Extended Data Figs. 5a and 6a $)^{16}$. As expected, following DR stimulation these circuits caused fast synaptic and slower extrasynaptic GABAA receptor mediated depolarizations of proprioceptive axons (termed sensoryevoked phasic PAD and tonic PAD, respectively ${ }^{16}$ ) that were blocked by GABAA antagonists, and mimicked by optogenetic activation of GABA axo neurons (Fig. 4a-d).

Like with direct GABAaxo activation, spike propagation failure was prevented by sensory-evoked phasic PAD, regardless of whether the failure was spontaneous (Figs. 4e-f,h), 5-HT-induced (Fig. 4h), or repetition-induced (Extended Data Fig. 6b-f). The latter is particularly important because sensory axons naturally fire at high rates, where they are vulnerable to spike failure (Fig. 2e-f). This action of phasic PAD was abolished by gabazine but not L655935, supporting a synaptic origin (Fig. 4h). Slow extrasynaptic GABAergic depolarization (tonic PAD; L655935-sensitive ${ }^{16}$ ) further facilitated spike propagation (Fig. 4g), especially as it built up with repeated DR stimulation (at $1 \mathrm{~Hz}$; Extended Data Fig. 5b). Cutaneous (Extended Data Fig. 5), proprioceptive (Extended Data Fig. 6) or mixed afferent (Fig. 4e-h) -evoked PAD all helped prevent spike failure.

In secure non-failing axon branches sensory-evoked PAD (or optogenetic GABAaxo activation) sped up the spikes and lowered their threshold (rheobase current; Fig. 3d and Extended Data Fig. 7a-d), as predicted from computer simulations (Extended Data Fig. 4e). Importantly, spike height was only slight reduced during PAD $(\sim 1 \%$ or $1 \mathrm{mV})$ indicating that nodal $\mathrm{GABA}_{\mathrm{A}}$ receptor conductances have minimal shunting action (Fig. 3 and Extend Data Fig. 7a-d).

\section{Failure of axon conduction to motoneurons and rescue by PAD.}

To quantify the overall failure of spikes to conduct from the DR to the sensory axon terminals we measured whether axon branches not conducting during failure were not refractory to subsequent stimulation with a microelectrode in the ventral horn (Extended Data Fig. 8). This method indicated that 
about $50-80 \%$ of sensory axons failed to conduct to their ventral terminals under resting conditions, especially in long axons, whereas sensory-evoked PAD decreased failure to $<30 \%$. Similar conclusions were reached by directly recording the extracellular afferent volley in the ventral horn produced by the spikes propagating from a DR stimulation to the motoneurons, which was consistently increased by PAD (Extended Data Fig. 9).

\section{Facilitation of sensory feedback to motoneurons by nodal GABA $\mathrm{A}_{\mathrm{A}}$ receptors.}

To examine the functional role of nodal GABA in regulating sensory feedback to motoneurons, we recorded monosynaptic excitatory postsynaptic potentials (EPSPs) from motoneurons in response to proprioceptive sensory axon stimulation (Fig. 5a). This EPSP was inhibited by optogenetically silencing GABA $_{\text {axo }}$ neurons with light in mice expressing archaerhodopsin-3 (Arch3, induced in GAD2 ${ }^{\mathrm{CreER} /+} ; \mathrm{R} 26^{\text {LSL-Arch3-GFP }}$ mice; abbreviated GAD2//Arch3, Fig. 5a-b,d), consistent with a tonic $\mathrm{GABA}_{\mathrm{A}}$ receptor tone facilitating spike transmission in axons. Likewise, the EPSP was reduced when sensory axon conduction was reduced by blocking endogenous GABAA receptor tone with antagonists, despite increasing motoneuron and polysynaptic reflex excitability (minimized with APV, Fig. 5c,d). $\mathrm{GABA}_{\mathrm{B}}$ antagonists slightly increased the EPSP, suggesting a tonic GABAв-mediated presynaptic inhibition (Fig. 5d), though much smaller than the tonic GABAA-mediated nodal facilitation that dominates when all GABA was reduced (in GAD2//Arch3 mice).

Consistent with nodal GABA $\mathrm{A}_{\mathrm{A}}$ receptors (and PAD) facilitating axon conduction, the monosynaptic EPSP was facilitated during, but not after, depolarizing proprioceptive axons (evoking PAD) with an optogenetic activation of GABAaxo neurons in GAD2//ChR2 mice (10 ms light conditioning stimulation; Fig. 5e-f). The EPSP was also facilitated by naturally activating GABAaxo neurons by a sensory conditioning stimulation, including with a conditioning stimulation of cutaneous and/or proprioceptive afferents (Extended Data Fig. 10a,b,e). The latter indicates that proprioceptive activity primes subsequent proprioceptive reflex transmission (self-facilitation). GABAA receptor antagonists (gabazine), but not GABAB antagonists (CGP55845), blocked the EPSP facilitation with sensory (Extended Data Fig. 10e) or light (Fig. 5f) conditioning.

The facilitation of the EPSP by conditioning-evoked PAD arose from axonal GABAA receptors, rather than from postsynaptic actions on the motoneurons, since it occurred with weak conditioning stimuli that produced only a transient background postsynaptic depolarization that terminated before the EPSP testing (at 60 ms; Figs. 5e, Extended Data Fig. 10b,g), followed by a slight hyperpolarization that if 
anything reduced the EPSP (shunting the synaptic current, Extended Data Fig. 10h). Increasing the DR conditioning intensity produced large background depolarizing conductances in the motoneurons during the EPSP testing, which led to postsynaptic inhibition of the EPSP (shunting inhibition; Extended Data Fig. 10d,g), masking the effect of nodal facilitation. Importantly, sometimes PAD itself induced afferent spikes (Extended Data Fig. 7e; termed DRR spikes), and following these spikes, the EPSP was always smaller than when these spikes were not present ( $n=8 / 8$ mice, not shown). This is because these DRR spikes themselves triggered EPSPs, leading to a post activation depression, as noted by Eccles ${ }^{9}$, and thus we minimized DRR activity by keeping the conditioning-evoked PAD small.

Sensory conditioning was particularly effective when it was repeated to mimic natural firing, which increased tonic PAD for minutes (Fig. 5g). This facilitated the EPSP for $\sim 3$ min after a brief fast DR repetition $(200 \mathrm{~Hz}, 0.5 \mathrm{~s}$ conditioning, Fig. 5i, Extended Data Fig. 10e, Tonic), and $\sim 1$ min after slower repetition $(0.1 \mathrm{~Hz}, 2$ min conditioning, Extended Data Fig. 10e, After effect), both long outlasting postsynaptic effects from each conditioning pulse $(<1 \mathrm{~s})$. This was blocked by L655708 or gabazine (Extended Data Fig. 10e).

\section{Increases in the probability of unitary EPSPs by nodal facilitation.}

We often noticed large all-or-nothing EPSPs (unitary EPSPs) spontaneously fluctuating on and off during repeated EPSP testing, leading to discrete large changes in the total EPSP size and time course (Fig. 5j-k). We thought this might be due to spontaneous branch point failures, rather than quantal changes in transmitter release that produce much smaller fluctuations ${ }^{35}$, as previously suggested ${ }^{28}$. Indeed, when we increased the axon conduction by activating the GABAaxo neurons and PAD (via a cutaneous conditioning train) the probability of unitary EPSPs occurring increased (Fig. 5k-1), and this sometimes recruited further large unitary EPSPs (Fig. 5k). In contrast, the size of the underlying unitary EPSP was not increased by this conditioning (Fig. 5j-1), ruling out decreases in terminal presynaptic inhibition or postsynaptic inhibition contributing to the increased overall EPSP (Fig. 5i,1).

\section{Facilitation of sensory feedback to motoneurons by GABA $_{a x o}$ neurons in awake mice.}

To determine whether GABAaxo neurons increase sensory feedback to motoneurons in awake mice we activated these neurons with light applied through a window chronically implanted over the spinal cord of GAD2//ChR2 mice (Fig. 6a), and assessed the monosynaptic reflex (MSR) recorded in tail muscles in response to nerve stimulation (counterpart of EPSPs). As expected, the MSR was facilitated by a conditioning light pulse, but only during, and not after, the expected time of phasic PAD induced on 
sensory axons (Fig. 6b-d,j). This light-induced facilitation occurred both at rest and when there was a background voluntary contraction, with the latter matched with and without light, again ruling out postsynaptic depolarization related differences in MSR (Fig. 6d). Light alone caused a brief pause in ongoing EMG ( $\sim 30 \mathrm{~ms}$ post-light; Fig. $6 \mathrm{~b})$, indicative of postsynaptic inhibition, which masked nodal facilitation at short intervals.

\section{Facilitation of sensory feedback to motoneurons during PAD in humans and rats}

Finally, to estimate the role of GABAaxo neurons in humans (and awake rats) we employed the sensoryevoked depolarization of proprioceptive axons by GABAaxo neurons (sensory-evoked PAD; Fig. 4), which is known to occur in humans ${ }^{36}$. For this we recorded the MSR in humans in the soleus muscle in response to tibial nerve stimulation (Fig. 7a). Increasing GABA axo neuron activity with a brief cutaneous stimulation increased the MSR (Fig 7bi, d and Fig. 6e-g) during a period consistent with nodal facilitation by PAD (30 - 200 ms post stimulation; in humans and rats, Fig. 7bii and Fig. 6j). We again kept the conditioning stimulation small enough to not change the background EMG or single motor unit (MU) firing (Fig. 7biii, Fig 6f-g) to rule out postsynaptic actions. When we instead increased PAD by a proprioceptive conditioning in humans (via muscle TA vibration) the soleus MSR was inhibited (for up to $200 \mathrm{~ms}$; Fig. 7ci-ii), as previously reported ${ }^{37}$. However, the vibration alone inhibited the ongoing MU discharge (Fig. 7ciii), implying that this MSR inhibition was caused in part by postsynaptic inhibition, rather than PAD-mediated presynaptic inhibition ${ }^{37}$.

Blocking GABAA receptor tone (in rats) decreased the MSR, at matched levels of background EMG (Fig. 6h), suggesting a spontaneous tonic PAD facilitating the MSR. In rats and humans repeated cutaneous conditioning stimulation (trains) to induce a buildup in this tonic PAD caused an associated buildup of the MSR that outlasted the conditioning and its postsynaptic actions by many seconds (after effect; Fig. 7d,e; Fig. 6i).

Importantly, the probability of a single MU contributing to the human MSR was increased by cutaneous conditioning (Fig. 7fi-ii). This occurred without an increase in the estimated EPSP amplitude or rise time (PSF; see Methods; Fig. 7Fiii) or change in the MU firing prior to the MSR testing (Fig. 7Fiv; motoneuron not depolarizing closer to threshold), consistent with an increased probability of unitary ESPSs, as in rats (Fig. 5). 


\section{Discussion}

Following the pioneering studies of Eccles on inhibition of the monosynaptic connection from sensory axons to motoneurons ${ }^{9}$, the concept of presynaptic inhibition of axon terminals has stood as a cornerstone of our understanding of mammalian brain and spinal cord function ${ }^{10}$. While presynaptic inhibition has never been directly confirmed in these sensory axons, recordings from invertebrate sensory axons have firmly established the idea that terminal GABAA receptor-mediated depolarizations (PAD) can cause conductance increases (shunts) or sodium channel inactivation that inhibit transmitter release ${ }^{22,24}$. Thus, our finding that $\mathrm{GABA}_{\mathrm{A}}$ receptors are located too far from the axon terminals to influence terminal depolarizations (relative to short $\lambda \mathrm{s}$ ) or cause presynaptic inhibition of transmitter release onto motoneurons (EPSPs not inhibited) had not been anticipated. Direct recordings from terminal boutons of other axon types in the mammalian brain (e.g. Calyx of Held) have shown that terminal $\mathrm{GABA}_{\mathrm{A}}$ or glycine receptors sometimes cause presynaptic inhibition, and at other times facilitate transmitter release, depending on their action on terminal calcium and potassium channels ${ }^{21,22,38}$. However, these terminal actions of GABA are very different from the actions of GABA on the nodes that we have uncovered, where GABAA receptors near sodium channels (nodes) and associated branch points help prevent conduction failure by bringing nodes closer to spike threshold. We found that without nodal GABA provided by $\mathrm{GABA}_{\text {axo }}$ neurons, spike transmission fails in many central branches of large myelinated sensory axons, leaving large silent branches, depending on the branching structure and prior history of activity (frequency). Thus, neuronal circuits that control $\mathrm{GABA}_{\mathrm{axo}}$ neurons serve to aid branch conduction via nodal facilitation. This concept of nodal facilitation may generalize to other large central axons (e.g. pyramidal cells) that are innervated by GABAergic neurons, branch extensively (and so may fail), and have depolarizing actions of GABA $^{20,22,39,40}$, allowing selective recruitment of specific axon branches and functional pathways, especially for high frequency firing.

Sensory driven $\mathrm{GABA}_{\mathrm{axo}}$ circuits (and PAD) are experimentally convenient, since they allow us to estimate how sensory transmission to motoneurons is modulated with GABA axo $_{\text {neuron activity in }}$ humans. Specifically, the expected PAD evoked by cutaneous conditioning is associated with a potent reflex facilitation in humans and awake rodents. This suggests that a substantial ongoing spike failure (prior to facilitation) that can be alleviated by GABAaxo activity (PAD). Indeed, we found that during PAD the probability of EPSPs occurring (and MU firing) is increased without changing the EPSP amplitude (estimated by PSFs in humans). The latter rules out changes in presynaptic inhibition with 
PAD that grades the EPSP size, including ruling out previous arguments that MSR facilitation by cutaneous conditioning is due to a removal of presynaptic inhibition ${ }^{41,42}$.

A pressing question that remains is how can nearly a century of research on sensory transmission and presynaptic inhibition be reconciled with GABA-mediated nodal facilitation and reflex facilitation (Table S1)? Sensory axon conduction failure has repeatedly been noted from indirect observations ${ }^{28-30}$, but GABAA receptors and PAD were previously thought to cause, rather than prevent, conduction failure $^{30}$, even though computer simulations showed physiological GABA levels unlikely to block spike propagation $^{34}$, as we confirmed. Furthermore, the fundamental assumption that GABAA receptors cause presynaptic inhibition that reduces transmitter release from sensory axons was from the outset circumspect, based mainly on the observation that a conditioning stimulation (on a flexor nerve) caused an inhibition of the MSR (evoked in extensor muscles) that was somewhat correlated to the time-course of PAD caused by this conditioning (in extensor afferents) ${ }^{9}$. However, in retrospect this PAD is too brief to account for the much longer (up to $1 \mathrm{~s}$ ) inhibition caused by this conditioning $9,11,43$, and GABAв receptor antagonists block much of this MSR inhibition ${ }^{17,43}$. This fits with GABAв receptors being at the terminals (Fig. 1) and primarily responsible for presynaptic inhibition in proprioceptive axons, as in other neurons ${ }^{21,22}$, though further study of GABAB receptor function is now needed. This predominant $\mathrm{GABA}_{\mathrm{B}}$ action in proprioceptive axon terminals does not rule out $\mathrm{GABA}_{\mathrm{A}}$-mediated presynaptic inhibition in other sensory axons that have dense terminal GABAA receptor expression, such as in cutaneous afferents ${ }^{16}$.

Anatomical studies have shown that GABAaxo neuron activation is inevitably accompanied by some postsynaptic inhibition, since most GABA axo contacts on afferent terminals also contact motoneurons, in a triad ${ }^{8,44}$. Indeed, we find that GABA axo neuron activation produces an inhibition of motoneurons (Fig. 6b) and associated MU firing (Fig. 7c) that masks, and at times overwhelms, the facilitation of the MSR by nodal GABAA receptors (as with muscle vibration). This postsynaptic inhibition was likely previously mistaken for presynaptic inhibition, though this needs to be re-examined ${ }^{37}$. The argument that presynaptic inhibition with conditioning should be evident from reductions in the EPSP without changing its time course ${ }^{45}$ now seems untenable, especially as unitary EPSPs differ markedly in shape and conditioning increases the number of unitary EPSPs contributing to the EPSP, as different axon branches are recruited (Fig. $5 \mathrm{k})^{28}$. 
Early on Barron and Matthews ${ }^{23}$ and later others ${ }^{7,16}$ established that sensory-evoked PAD (or lightevoked) excites axons by directly inducing spiking, including spikes in the sensory axons mediating the MSR itself, raising a further contradiction with presynaptic inhibition. While these PAD-triggered spikes only sometimes fully propagate antidromically out the $\mathrm{DR}^{46}$, they are more likely to conduct orthodromically ${ }^{16}$ where they activate the motoneurons ${ }^{7,9,47}$, making these axons and their motoneuron synapse refractory to subsequent testing 9 . This contributes to a long-lasting post-activation depression of the MSR pathway that is GABAA-mediated (sensitive to GABAA antagonists, like PAD) and is thus readily mistaken for GABAA-mediated presynaptic inhibition ${ }^{7,17,18,37}$.

Functionally, nodal facilitation and regulation of branch point failure by GABAaxo-driven $\mathrm{GABA}_{\mathrm{A}}$ receptors acts like a global switching system that recruits entire silent sensory or motor circuits. This works in concert to terminal presynaptic inhibition (including $\mathrm{GABA}_{\mathrm{B}}$ receptor action) that locally fine tunes reflex gains, to optimize the stability and compliance of movement ${ }^{3,4,7}$. The direct activation of GABAaxo neurons (and PAD) by cortical (CST) and spinal (CPG) circuits $^{12,13,48}$, and inhibition by the brainstem $(5-\mathrm{HT})^{33,49}$, suggests that nodal facilitation is under explicit central control during reaching and locomotion (Fig. 2). The widespread action of PAD (occurring simultaneously over many spinal segments) $)^{11,16}$ implies that nodal facilitation acts over large regions of the spinal cord to ready sensory axons for action during cortical, spinal or sensory evoked activity, reminiscent of the Jendrassik maneuver ${ }^{50}$, ensuring that adequate sensory feedback aids postural stability and walking. More generally, our results imply that each axonal branch point has the capacity to function separately (like separate neurons), depending on their GABAergic innervation, increasing the complexity of sensory processing in the spinal cord. 


\section{Methods}

\section{Adult mice, rats and humans used.}

Recordings were made from large proprioceptive group Ia sensory afferents, GABAergic neurons, motoneurons and muscles in adult mice (2.5 - 6 months old, both female and male equally; strains detailed below) and rats (3 - 8 months old, female only, Sprague-Dawley). All experimental procedures were approved by the University of Alberta Animal Care and Use Committee, Health Sciences division. Recordings were also made from the soleus muscle of neurologically intact adult humans (female and male equally), aged 21 to 58, with written informed consent prior to participation. Experiments were approved by the Health Research Ethics Board of the University of Alberta (Protocols 00023530 and 00076790) and conformed to the Declaration of Helsinki. No effects of sex were noted and data from both sexes were combined for analysis.

\section{Mice used for optogenetics and imaging.}

We evaluated GABAergic neurons in a strain of mice with Cre expressed under the endogenous Gad2 promotor region. Gad2 encodes the Glutamate decarboxylase 2 enzyme GAD2 (also called GAD65), which is unique to axoaxonic contacting GABAergic neurons that project to the ventral horn, whereas all GABAergic neurons express GAD1 ${ }^{6}$. These GAD2 ${ }^{+}$neurons were activated or inhibit optogenetically using channelrhodopsin-2 (ChR2) $)^{51,52}$ or archaerhodopsin-3 (Ach3 $)^{53,54}$, respectively. The following mouse strains were employed (Supplementary Table 2):

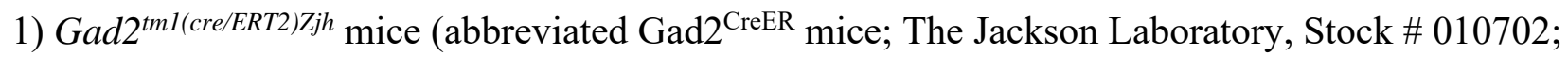
$\mathrm{CreER}^{\mathrm{T} 2}$ fusion protein expressed under control of the endogenous Gad2 promotor $)^{55}$,

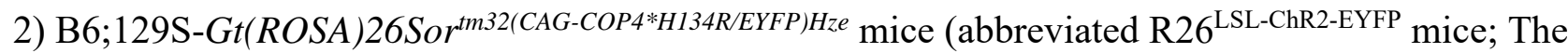
Jackson Laboratory, Stock \# 012569; ChR2-EYFP fusion protein expressed under the R26::CAG promotor in cells that co-express Cre because a loxP-flanked STOP cassette, LSL, prevents transcription of the downstream ChR2-EYFP gene) ${ }^{56}$,

3) B6.Cg-Gt(ROSA)26Sor ${ }^{\text {tm14(CAG-tdTomato)Hze and B6.Cg-Gt(ROSA)26Sor }}{ }^{\text {tm9(CAG-tdTomato)Hze mice }}$ (abbreviated R26 $6^{\text {LSL-tdTom }}$ mice; The Jackson Laboratory, Stock \# 007914 and \#007909; tdTomato fluorescent protein expressed under the R26::CAG promotor in cells that co-express Cre $)^{57}$,

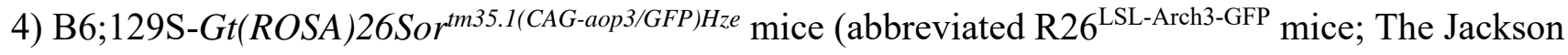
Laboratory Stock \# 012735; Arch3-GFP fusion protein expressed under the R26::CAG promotor in cells that co-express $\mathrm{Cre})^{56}$, and 
5) B6;129S-Slc17a7tm1.1(cre)Hze mice (abbreviated VGLUT1 ${ }^{\text {Cre }}$ mice; The Jackson Laboratory, Stock \# 023527; Cre protein expressed under control of the endogenous Vglut1 promotor; kindly donated by Dr. Francisco J. Alvarez) ${ }^{58}$.

Heterozygous GAD2 ${ }^{\mathrm{CreER}}$ mice (i.e., GAD2 ${ }^{\mathrm{CreER} /+}$ mice) were crossed with homozygous reporter strains to generate GAD2 $2^{\mathrm{CreER} /+} ; \mathrm{R} 26^{\mathrm{LSL}-\mathrm{ChR} 2-\mathrm{EYFP}}, \mathrm{GAD} 2^{\mathrm{CreER} /+} ; \mathrm{R} 26^{\mathrm{LSL}-\mathrm{tdTom}}$ and GAD2 ${ }^{\mathrm{CreER} /+}$; R26 $6^{\mathrm{LSL}-\mathrm{Arch} 3-\mathrm{GFP}}$ mice that we abbreviate: GAD2//ChR2, GAD2//tdTom and GAD2//Arch3 mice. Offspring without the GAD2 ${ }^{\mathrm{CreER}}$ mutation, but with the effectors ChR2, Arch3 or tdTom were used as controls. We also used mice bred by crossing homozygous VGLUT1 ${ }^{\text {Cre }}$ mice with R2 $6^{\text {lsl-tdTom }}$ reporter mice to obtain mice with VGLUT1 labelled sensory axons ${ }^{59}$.

CreER is an inducible form of Cre that requires tamoxifen to activate ${ }^{60}$, which we applied in adult mice to prevent developmental issues of earlier induction of Cre. Specifically, mice were injected at 4 - 6 weeks old with two doses of tamoxifen separated by two days, and studied $>1$ month later, long after washout of tamoxifen. Each injection was $0.2 \mathrm{mg} / \mathrm{g}$ wt (i.p.) of tamoxifen dissolved in a corn oil delivery vehicle (Sigma C8267). These tamoxifen-treated mice were denoted GAD2//ChR2+ and GAD2//Arch3+, and non treated mice were used as controls and denoted GAD2//ChR2- and GAD2//Arch2-. For all mice, genotyping was performed according to the Jackson Laboratories protocols by PCR of ear biopsies using primers specific for the appropriate mutant and wild type alleles for each of the mouse lines (see Key Resources Table for primer details).

\section{Ex vivo recording from axons and motoneurons in whole adult spinal cords.}

Mice or rats were anaesthetized with urethane (for mice $0.11 \mathrm{~g} / 100 \mathrm{~g}$, with a maximum dose of $0.065 \mathrm{~g}$; and for rats $0.18 \mathrm{~g} / 100 \mathrm{~g}$, with a maximum dose of $0.45 \mathrm{~g}$ ), a laminectomy was performed, and then the entire sacrocaudal spinal cord was rapidly removed and immersed in oxygenated modified artificial cerebrospinal fluid (mACSF), as detailed previously ${ }^{61-63}$. This preparation is particularly useful as the small sacrocaudal spinal cord is the only portion of the adult spinal cord that survives whole ex vivo, allowing axon conduction to be assessed along large distances. Further, this segment of cord innervates the axial muscles of the tail that are readily assessable for reflex recording in awake animals, and has proven to be a useful model of motor function in normal and injured spinal cords ${ }^{62,64}$. Spinal roots were removed, except the sacral S3, S4 and caudal Ca1 ventral and dorsal roots on both sides of the cord. After 1.5 hours in the dissection chamber (at $20^{\circ} \mathrm{C}$ ), the cord was transferred to a recording chamber containing normal ACSF (nACSF) maintained at $23-32^{\circ} \mathrm{C}$, with a flow rate $>3 \mathrm{ml} / \mathrm{min}$. A one-hour 
period in nACSF was given to wash out the residual anaesthetic prior to recording, at which time the nACSF was recycled in a closed system. The cord was secured onto tissue paper at the bottom of a rubber (Silguard) chamber by insect pins in connective tissue and cut root fragments. The dorsal surface of the cord was usually oriented upwards when making intracellular recording from afferents in the dorsal horn, whereas the cord was oriented with its left side upwards when making recordings from motoneurons or afferent terminals in the ventral horn. The laser beam used for optogenetics was focused vertically downward on the GAD2 neurons, as detailed below.

\section{Optogenetic regulation of $\mathrm{GABA}_{\mathrm{axo}}$ neurons.}

The GAD2//ChR2 or GAD2//Arch3 mice were used to optogenetically excite or inhibit GAD2+ neurons (with 447 nm D442001FX and 532 nM LRS-0532-GFM-00200-01 lasers from Laserglow Technologies, Toronto), respectively, using methods we previously described ${ }^{65}$. Light was derived from the laser passed through a fibre optic cable (MFP_200/220/900-0.22_2m_FC-ZF1.25 and MFP_200/240/3000-0.22_2m_FC-FC, Doric Lenses, Quebec City) and then a half cylindrical prism the length of about two spinal segments ( $8 \mathrm{~mm} ; 3.9 \mathrm{~mm}$ focal length, Thor Labs, Newton, USA,), which collimated the light into a narrow long beam $(200 \mu \mathrm{m}$ wide and $8 \mathrm{~mm}$ long). This narrow beam was focused longitudinally on the left side of the spinal cord roughly at the level of the dorsal horn, to target the epicentre of GABAaxo neurons, which are dorsally located (Fig. 3). ChR2 rapidly depolarizes neurons ${ }^{52}$, and thus we used 5-10 ms light pulses to activate GABAaxo neurons, as confirmed by direct recordings from these neuron (see below). Light was always kept at a minimal intensity, 1.1x $\mathrm{T}$, where $\mathrm{T}$ is the threshold to evoke a light response in sensory axons, which made local heating from light unlikely. Arch3 is a proton pump that is activated by green light, leading to a hyperpolarization and slowly increased $\mathrm{pH}$ (over seconds), both of which inhibit the neurons ${ }^{52,66}$. Thus, we used longer light pulses ( 200 ms) to inhibit GABAaxo neurons.

To directly confirm the presence of functional GAD2 expression in neurons (GABA axo neurons) we recorded from them with similar methods and intracellular electrodes used to record from motoneurons (see below). Electrodes were advanced into these cells through the dorsal horn (with the dorsal surface oriented upwards), and their identity established by a direct response to light activation of the ChR2 construct (5 - $10 \mathrm{~ms}$ light pulse, $447 \mathrm{~nm}, \mathrm{~mW}$; in GAD2//ChR2 mice), without a synaptic delay $(<1 \mathrm{~ms})$ and continued light response after blocking synaptic transmission.

\section{Dorsal and ventral root stimulation.}


Dorsal and ventral roots (DR and VR) were mounted on silver-silver chloride wires above the nASCF of the recording chamber and covered with grease (a 3:1 mixture of petroleum jelly and mineral oil) for monopolar stimulation $16,64,67$. This grease was surrounded by a more viscous synthetic high vacuum grease to prevent oil leaking into the bath flow. Bipolar stimulation was also used at times to reduce the stimulus artifact during recording from ventral roots (detailed below). Roots were stimulated with a constant current stimulator (Isoflex, Israel) with short pulses $(0.1 \mathrm{~ms})$. Note that proprioceptive afferents are selectively activated by low intensity DR stimulation $(1.1-1.5 \mathrm{x}$ threshold, $\mathrm{T})$ and cutaneous afferents are additionally activated by higher intensity DR stimulation $(2-3 \times T)$. DRs were dissected to be as long as possible, and the distal end of this root was stimulated, so it was $\sim 20 \mathrm{~mm}$ way from the spinal cord. In this way the DR stimulation site itself (at wire, and threshold for stimulation) could not be affected by axonal depolarizations in the spinal cord, since dorsal root potentials from spinal events (PAD) are only observed very close to the cord (within a few mm, see below), and drop exponentially in size with distance ${ }^{16}$.

\section{Intracellular recording from sensory axon branches in the dorsal horn.}

Electrode preparation and amplifier. Recording from fine afferent collaterals in the spinal cord without damaging them or disturbing their intracellular milieu required specialized ultra-sharp intracellular electrodes modified from those we developed for motoneuron recording ${ }^{61}$. That is, glass capillary tubes (1.5 mm and $0.86 \mathrm{~mm}$ outer and inner diameters, respectively; with filament; 603000 A-M Systems;

Sequim, USA) were pulled with a Sutter P-87 puller (Flaming-Brown; Sutter Instrument, Novato, USA) set to make bee-stinger shaped electrodes with a short relatively wide final shaft $(\sim 1 \mathrm{~mm})$ that tapered slowly from 30 to $3 \mu \mathrm{m}$ over its length, and then abruptly tapered to a final tip over the final $20 \mu \mathrm{m}$ length. The tip was subsequently bevelled to a $100 \mathrm{~nm}$ hypodermic-shaped point, as verified with electron microscope images (Harvey et al. 2006). This very small tip and wide shaft gave a combination of ease of penetrating axons in dense adult connective tissue, and good current-passing capabilities to both control the potential and fill the axons with neurobiotin. Prior to beveling, electrodes were filled through their tips with $2 \mathrm{M} \mathrm{K}$-acetate mixed with varying proportions of $2 \mathrm{M} \mathrm{KCl}$ (to make intracellular Cl- concentrations ranging of $0,100,500$, and $1000 \mathrm{mM}$ ) or $500 \mathrm{mM} \mathrm{KCl}$ in 0.1 Trizma buffer with 5 10\% neurobiotin (Vector Labs, Birmingame, USA). They were then beveled from an initial resistance of 40 - $150 \mathrm{M} \Omega$ to 30 - $40 \mathrm{M} \Omega$ using a rotary beveller (Sutter BV-10). GABAergic chloride-mediated potentials $(\mathrm{PAD})$ were the same with different concentrations of $\mathrm{KCl}$, without passing large amounts of negative current, as we have previously detailed ${ }^{16}$, indicating that the ultra-sharp tips impeded passive fluid exchange between the electrode and intracellular milieu, with in particular electrode $\mathrm{Cl}^{-}$not 
affecting the axon; thus, recordings were mostly made with electrodes with $1 \mathrm{M} \mathrm{K}$-acetate and $1 \mathrm{M}$ $\mathrm{KCl}$, when not filling cells with neurobiotin.

Intracellular recording and current injection were performed with an Axoclamp2B amplifier (Axon Inst. and Molecular Devices, San Jose, USA). Recordings were low pass filtered at $10 \mathrm{kHz}$ and sampled at $30 \mathrm{kHz}$ (Clampex and Clampfit; Molecular Devices, San Jose, USA). Sometimes recordings were made in discontinuous-single-electrode voltage-clamp (gain $0.8-2.5 \mathrm{nA} / \mathrm{mV}$; for Ca PICs) or discontinuouscurrent-clamp modes (switching rate $7 \mathrm{kHz}$ ), as indicated (the latter only when injecting current, for example during recording of input resistance or the voltage dependence of spikes).

Axon penetration. Electrodes were advanced into myelinated afferents with a stepper motor (Model 2662, Kopf, USA, $10 \mu \mathrm{m}$ steps at maximal speed, $4 \mathrm{~mm} / \mathrm{s}$ ), usually at the boundary between the dorsal columns and dorsal horn gray matter. Extracellular tissue (especially myelin in the white matter) often impeded and blocked the electrode tip following a forward step, as determined by an increase in resistance to small current pulses passed from the tip of the electrode $(20 \mathrm{~ms},-0.3 \mathrm{nA}, 1 \mathrm{~Hz})$, and this was cleared with a brief high frequency current (from capacitance overcompensation buzz) and moving backwards slowly, the latter which helped prevent tissue dimpling. Prior to penetrating afferents, we recorded the extracellular (EC) afferent volley following dorsal root (DR) stimulation (0.1 ms pulses, $3 \times \mathrm{T}$, threshold, where $\mathrm{T}=\sim 3 \mathrm{uA}$, repeated at $1 \mathrm{~Hz}$ ), to determine the minimum latency and threshold of afferents entering the spinal cord. The group Ia afferent volley occurs first with a latency of $0.5-1.0$ $\mathrm{ms}$, depending on the root length (which were kept as long as possible, $10-20 \mathrm{~mm}$ ), corresponding to a conduction velocity of about $16-24 \mathrm{~m} / \mathrm{s}$, as previously described for in vitro conduction at $23 \mathrm{C}^{16,68}$. When a forward step penetrated an axon, small slow movements were made to stabilize the recordings. Penetrations were usually in the myelinated portion of the axon between nodes, rather than at nodes, because the chance of penetrating a node is low since they only make up a small fraction of the total axon length (Fig. 1). The spikes from the two nodes adjacent to the electrode were readily detected separately when testing for the spike threshold with current injection pulses (20 ms; rheobase test), because just at threshold the current sometimes evoked a spike from just one node and not the other, which usually halved the total spike height, consistent with the penetration being about halfway between the two nodes.

Proprioceptive afferent identification. Upon penetration, afferents were identified with direct orthodromic spikes evoked from DR stimulation. We focused on the lowest threshold (T) 
proprioceptive group Ia afferents, identified by their direct response to DR stimulation, very low threshold $(<1.5 \mathrm{x} \mathrm{T})$, short latency (group Ia latency, coincident with onset of afferent volley), and antidromic response to ventral horn afferent terminal microstimulation $(\sim 10 \mu \mathrm{A}$ stimulation via tungsten microelectrode to activate Ia afferent terminals; tested in some afferents, detailed below ${ }^{16}$. Clean axon penetrations without injury occurred abruptly with a sharp pop detected on speakers attached to the recorded signal, the membrane potential settling rapidly to near $-70 \mathrm{mV}$, and $>70 \mathrm{mV}$ spikes readily evoked by DR stimulation or brief current injection pulses (1-3 nA, $20 \mathrm{~ms}, 1 \mathrm{~Hz})$. Sensory axons also had a characteristic $>100 \mathrm{~ms}$ long depolarization following stimulation of a dorsal root (primary afferent depolarization, PAD, at 4 - 5 ms latency, detailed below) and short spike afterhyperpolarization (AHP $\sim 10 \mathrm{~ms}$ ), which further distinguished them from other axons or neurons. Injured axons had higher resting potentials $(>-60 \mathrm{mV})$, poor spikes $(<60 \mathrm{mV})$ and low resistance (to current pulse; $\mathrm{R}_{\mathrm{m}}<10 \mathrm{M} \Omega$ ) and were discarded.

Quantification of spike conduction failure in the dorsal horn: failure potentials (FPs). Sometimes healthy intracellular penetrations were made into a sensory axon branch (e.g. $<-60 \mathrm{mV}$ rest, large PAD), but dorsal root stimulation did not evoke a full spike, even though a full $>60 \mathrm{mV}$ spike could be readily evoked by intracellular current injection. Instead, DR stimulation evoked a partial spike at the latency and threshold of group Ia afferents, indicating that this was a branch of a Ia afferent that failed to fully conduct spikes to the electrode, with only the passively attenuated spike from the last node to spike prior to conduction failure recorded at the electrode (failure potential, FP; also referred to as electronic residue by Luscher ${ }^{69}$ ). The size of the FP reflected how far away the spike failure occurred, with spatial attenuation corresponding to a space constant of about $90 \mu \mathrm{m}$ (see Results), and so FPs became exponentially smaller with distance from failure and undetectable when many mm away (nodes separated by about $50 \mu \mathrm{m})$. Occasionally axons were penetrated with undetectable DR evoked spikes or FPs, but otherwise they had characteristics of a Ia afferent (PAD, $\mathrm{R}_{\mathrm{m}}$ similar). These were likely afferents with FPs too distal to detect, but were usually excluded from the main analysis to avoid ambiguity, though this underestimates the incidence of failure. However, some of these axons exhibited short latency, low threshold DR spikes when depolarized by a prior DR stimulation (PAD) of an adjacent DR, in which case they were unequivocally Ia afferents and included in the analysis (Fig. 4f).

Both during extracellular and intracellular recording the group Ia afferent volley (small negative field) was observed as the first event after DR stimulation (the latter subthreshold to a spike), though this was usually small in relation to intracellular events and ignored. However, this was sometimes removed 
from the intracellular record by subtracting the extracellular potential recorded just outside the same axon to determine the actual transmembrane potential ${ }^{16}$. This was necessary to see the very smallest FPs following DR stimulation in some afferents, as the negative volley from other nearby afferents obscured the FPs.

After quantifying the axons spikes and conduction failures (FPs) under resting conditions, we then examined the changes in spike conduction with changes in membrane potential induced by either directly injecting current into axons or inducing GABA-mediated changes in membrane potential by pharmacological methods, optogenetic methods (activating ChR2 on GABA axo neurons to induce PAD) or more naturally evoking PAD with a DR stimulation.

Neurobiotin filling of axons. Some of the proprioceptive afferents that we recorded intracellularly were subsequently filled with neurobiotin by passing a very large positive 2 - 4 nA current with $90 \%$ duty cycle (900 ms on, $100 \mathrm{~ms}$ off) for $10-20 \mathrm{~min}$. The identity of group Ia proprioceptive afferents were then confirmed anatomically by their unique extensive innervation of motoneurons ${ }^{16}$. Prior to penetrating and filling axons with neurobiotin filled electrodes, a small negative holding current was maintained on the electrodes to avoid spilling neurobiotin outside axons.

\section{Quantification of spike conduction failure in the ventral horn}

Wall's method. To measure whether spikes fail during propagation to their fine terminals in the ventral horn we examined whether failed axon segments were relatively less refractory to activation after spike conduction failure, using a double pulse method adapted from Wall ${ }^{30,70}$. The essence of the method is that after DR activation all nodes that generate spikes become relatively refractory for a few ms, whereas nodes that fail to spike are not refractory to activation. Thus, a microelectrode placed near these failing nodes more readily activates them if they fail rather than generate spikes with DR stimulation and orthodromic conduction. For this we placed a tungston microelectrode (12 $\mathrm{M} \Omega$, \#575400, A-M Systems, Sequim, USA) in the ventral horn near the axons terminals on motoneurons, to activate the branches/nodes of the axon projecting to the motoneuron that may have failed (VH stimulation).

Spikes from VH or DR stimulation were recorded intracellularly in a proprioceptive axon penetrated in the dorsal columns directly above the VH stimulation site or in an adjacent segment, with two combinations of double axon stimulations. First, we applied two rapidly repeated VH stimuli (VH 
doublet; two $0.1 \mathrm{~ms}$ pulses) at a $\sim 4 \mathrm{~ms}$ interval to make the axon relatively refractory to stimulation and determine both the threshold current to activate the first spike ( $T_{V H 1}$, with VH1 stimulation) and the higher threshold current to overcome this the inactivation and generate a second spike ( $T_{V H 2}$, with $\mathrm{VH} 2$ stimulation). Second, we repeated this double spike activation, but with the first activation from a suprathreshold DR stimulation (at 1.5x DR threshold) and the second from a VH stimulation at the $T_{V H 2}$ intensity from B (DR-VH pair). In this case the VH stimulation readily activates the axon spike if the orthodromic DR evoked spike does not propagate to the ventral horn, leaving the silent portion of the axon non refractory. Accordingly, we also determined the threshold current to activate the $\mathrm{VH}$ after the DH in this arrangement (termed $T_{D R, V H}$ ), which was lower than $T_{V H 2}$. For comparison to the spike inactivation with VH doublets, we adjusted the DR-VH pair timing slightly so that the pairs of spikes (or expected spikes, at vertical lines) are separated by the same interval ( $\sim \mathrm{ms}$ ) when they reach the recording site, to compensate for DR conduction delays. The putative spike failure with DR stimulation happens at a node somewhere between the recording site and the $\mathrm{VH}$, because we only studied axons that securely conducted single DR pulses to the recording site, and thus failure was not directly visible.

We quantified the spike failure based on the following considerations: If the DR-evoked spike entirely fails to propagate to the $\mathrm{VH}$, then the threshold for subsequently activating the ventral horn $\left(T_{D R, V H}\right)$ should be the same as the threshold without any prior activation $\left(T_{V H 1}=T_{D R, V H}\right)$, whereas if it does not fail, then the threshold for activating the ventral horn should be the same as with a VH $\operatorname{doublet}\left(T_{V H 2}=\right.$ $\left.T_{D R, V H}\right)$. In between these two extreme scenarios, the DR evoked spike may only partially fail to propagate spikes to the ventral horn (by only some of its branches failing or conducting only partially to the $\mathrm{VH}$ ); in this case $T_{D R, V H}$ should be between $T_{V H 1}$ and $T_{V H 2}$, with the difference $T_{V H 2}-T_{V H 1}$ representing the range of possible thresholds between full failure and full conduction. Thus, overall the failure was quantified as: Conduction failure $=\left(T_{V H 2}-T_{D R, V H}\right) /\left(T_{V H 2}-T_{V H 1}\right) \times 100 \%$, which is $100 \%$ at full failure and $0 \%$ with no failure. This estimate is predicated on the assumption that the failed spikes are only relatively refractory to conduction and increased stimulation can overcome this failure, which is reasonable for the interspike intervals we used, and means that the computed \% failure reflects the number of nodes that failed to spike, with more dorsal branch point failures giving more failed nodes. On the other hand, we used interspike intervals that were short enough for the DR stimulation not to evoke PAD that affected the subsequent spike threshold $(\sim 4 \mathrm{~ms})$, in contrast to the longer intervals where PAD can help DR doublet firing (DR-DR in Extended Data Fig. 6, 5 - $10 \mathrm{~ms}$ ). 
Extracellular recording from sensory axon terminals. To directly record spike conduction in proprioceptive afferent terminal branches in the $\mathrm{VH}$ we used our intracellular glass pipette electrode $(\sim 30 \mathrm{M} \Omega)$ positioned just outside these axons (extracellular, EC), to avoid penetration injury in these fine axon branches. The DR was stimulated near threshold (1.1xT) to evoke the EC response in a few single axons near the electrode, and many trials were averaged to remove noise from these small signals $\left(20-50\right.$ trials at $3 \mathrm{~s}$ intervals). The EC field was multiphasic as previously described for other axons ${ }^{71-}$ ${ }^{73}$, with a small initial positive field resulting from passively conducted axial current from sodium spikes at distant nodes (closer to the DR; outward current at electrode), some of which fail to propagate spikes to the $\mathrm{VH}$ recording site, making this field a measure of conduction failure ${ }^{71,73}$. Following this, a larger negative field arises, resulting from spikes arising at nodes near the electrode (inward current), making this negative field a measure of secure conduction. A relatively large stimulus artifact is present prior to these fields, due to the small size of the EC fields themselves, and we truncated this.

We conducted three control experiments to confirm the relation of these EC fields to spike conduction. First, in the dorsal horn where we can readily intracellularly record from large proprioceptive axon branches, we compared intracellular (IC) recordings from axons to EC recordings just outside the same axon, to confirm that the DR evoked spike (IC) arrives at about the time of the negative EC field. Second, we locally applied TTX to the DR near the recording site (10 $\mu$ l bolus of $100 \mu \mathrm{M}$ TTX over DR) which eliminated the negative field and left only the initial positive field, confirming that the positive field is from distal nodes upstream of the TTX block, and generated by passive axial current conduction. This is important, since some investigators have argued on theoretical grounds that the positive field can instead result from the closed end electrical properties of axons at their terminals ${ }^{74}$, rather than spike failure, though others have refuted this ${ }^{73}$. Finally, we improved nodal spike conduction by reducing the divalent cations $\mathrm{Mg}^{++}$and $\mathrm{Ca}^{++}$in the bath medium, since divalent cations normally cause a gating or guarding action on the sodium channel, the latter by one charge binding to the membrane and the other raising the local extracellular positive charge, and overall raising the local voltage drop across the channel and its spike threshold ${ }^{75}$. This decreased the failure-related initial positive field and increased the main EC negative field, indicating improved conduction, and again confirming the use of these fields as measures of conduction, similar to previous conclusions for the motor endplate ${ }^{71}$ and mathematical consideration of axon cable properties ${ }^{76}$.

To quantify the EC fields we estimated the overall conduction to the recording site as: 
Conduction Index $=n f /(n f+p f) \times 100 \%$, where $\mathrm{pf}$ and $\mathrm{nf}$ are the positive and negative EC field amplitudes. This conduction index approaches $100 \%$ for full conduction ( $\mathrm{pf} \sim=0$ ) and $0 \%$ for no conduction $(n f=0)$. The absolute EC field potential amplitudes are highly variable between different recordings sites, and thus are difficult to quantify across animals and sites, whereas this ratio of field amplitudes ( $\mathrm{nf} /(\mathrm{nf}+\mathrm{pf}))$ eliminates the variability, and can effectively be viewed as a normalization of the negative field (nf) by the total field peak-to-peak size $(\mathrm{nf}+\mathrm{pf})$.

\section{Intracellular recording from motoneurons.}

The same intracellular glass electrode, stepper motor and amplifier used for recording sensory axons were used for intracellular recording from motoneurons, except that the electrodes were bevelled to a lower resistance $(30 \mathrm{M} \Omega)$. The electrode was advanced into motoneurons with fast $2 \mu \mathrm{m}$ steps and brief high frequency currents (capacitance overcompensation) guided by audio feedback from a speaker. After penetration, motoneuron identification was made with antidromic ventral root stimulation, and noting ventral horn location, input resistance and time constant ( $>6 \mathrm{~ms}$ for motoneurons) ${ }^{62}$. The monosynaptic excitatory postsynaptic potentials (EPSPs) and associated currents (EPSCs) were measured in motoneurons following stimulation of dorsal roots (at 1.1- $1.5 \mathrm{xT}, 0.1 \mathrm{~ms}, 3-10 \mathrm{~s}$ trial intervals). These were identified as monosynaptic by their rapid onset (first component), lack of variability in latency $(<1 \mathrm{~ms}$ jitter $)$, persistence at high rates $(10 \mathrm{~Hz})$ and appearance in isolation at the threshold for DR stimulation $(<1.1 \times \mathrm{xT})$, unlike polysynaptic EPSPs which varying in latency, disappear at high rates, and mostly need stronger DR stimulation to activate.

\section{Dorsal and ventral root grease gap recording.}

In addition to recording directly from single proprioceptive axons and motoneurons, we employed a grease gap method to record the composite intracellular response of many sensory axons or motoneurons by recording from dorsal and ventral roots, respectively, as previously detailed for similar sucrose and grease gap methods, In this case, a high impedance seal on the axon reduces extracellular currents, allowing the recording to reflect intracellular potentials ${ }^{16,76-78}$. We mounted the freshly cut roots onto silver-silver chloride wires just above the bath, and covered them in grease over about a 2 $\mathrm{mm}$ length, as detailed above for monopolar recordings. Return and ground wires were in the bath and likewise made of silver-silver chloride. Specifically for sensory axons, we recorded from the central ends of dorsal roots cut within about 2 - $4 \mathrm{~mm}$ of their entry into the spinal cord, to give the compound potential from all afferents in the root (dorsal roots potential, DRP), which has previously been shown to correspond to PAD, though it is attenuated compared to the intracellular recordings of $\mathrm{PAD}^{16}$. The 
signal attenuation has two reasons. First the voltage PAD is attenuated along the length of nerve in the bath, as detailed in the next paragraph. Second, the grease does not completely remove the extracellular fluid around the nerve, even though we deliberately allowed the nerve to dry for a few seconds before greasing, and this causes a conductance that shunts or short circuits the recorded signal, reducing it by about half ${ }^{71,78}$. For optogenetic experiments we additionally added silicon carbide powder (9\% wt, Tech-Met, Markham) to the grease to make it opaque to light and minimize light induced artifactual current in the silver-silver chloride recording wire during optogenetic activation of ChR2 (detailed below). Likewise, we covered our bath ground and recording return wires with a plastic shield to prevent stray light artifacts. The dorsal root recordings were amplified (2,000 times), high-pass filtered at $0.1 \mathrm{~Hz}$ to remove drift, low-pass filtered at $10 \mathrm{kHz}$, and sampled at $30 \mathrm{kHz}$ (Axoscope 8; Axon Instruments/Molecular Devices, Burlingame, CA).

These grease gap recordings of PAD on sensory afferents reflect only the response of largest diameter axons in the dorsal root, mainly group I proprioceptive afferents, because of the following considerations. First, the largest axons in peripheral nerves have a nodal spacing of about $1 \mathrm{~mm}^{79,80}$, and length constants $\lambda_{\mathrm{s}}$ are estimated to be similar, at about $1-2$ times the nodal spacing ${ }^{81}$, Further, in our recordings we were only able to get the grease to within about $2 \mathrm{~mm}$ of the spinal cord. Thus, the centrally generated signal (PAD) is attenuated exponentially with distance $x$ along the axon length in the bath $(\mathrm{x}=2 \mathrm{~mm})$. This is proportional to $\exp \left(-\mathrm{x} / \lambda_{\mathrm{s}}\right)\left(\operatorname{see}^{76}\right)$, which is $1 / \mathrm{e}^{2}=0.11$ for $\mathrm{x}=2 \lambda_{\mathrm{s}}$, as is approximately the case here. This makes a central PAD of about $4 \mathrm{mV}$ appear as a $\sim 0.4 \mathrm{mV}$ potential on the root recording (DRP, 10 times smaller), as we previously reported ${ }^{16}$. Furthermore, the nodal spacing and $\lambda \mathrm{s}$ decrease linearly with smaller axon diameters ${ }^{76,79}$, making the voltages recorded on the smaller afferents contribute to much less of the compound root potential (halving the diameter attenuates PAD instead by $1 / \mathrm{e}^{4}$ or 0.012 , which is $99 \%$ attenuation). Finally, unmyelinated sensory axons attenuate voltages over a much shorter distance than myelinated axons, since that membrane resistance $(R m)$ drops markedly without myelin and $\lambda_{\mathrm{s}}$ is proportional to $\sqrt{R m / R i}$ (where $R i$ is axial resistance; Stein 1980). Thus, any centrally generated change in potential in these small axons is unlikely to contribute to the recorded signal $2 \mathrm{~mm}$ away.

The composite EPSPs in many motoneurons were likewise recorded from the central cut end of ventral roots mounted in grease (grease gap), which has also previously been shown to yield reliable estimates of the EPSPs (though again attenuated by the distance from the motoneurons) ${ }^{82}$. The monosynaptic EPSPs were again identified as monosynaptic by their rapid onset (first component, $\sim 1 \mathrm{~ms}$ after afferent 
volley arrives in the ventral horn; see below), lack of variability in latency $(<1 \mathrm{~ms}$ jitter $)$, persistence at high rates $(10 \mathrm{~Hz})$ and appearance in isolation at the threshold for DR stimulation $(<1.1 \mathrm{xT})$, unlike polysynaptic reflexes which varying in latency, disappear at high rates, and mostly need stronger DR stimulation to activate.

\section{Analysis of synaptic responses in sensory axons (PAD) and motoneurons (EPSPs).}

When we recorded from sensory axons of an associated dorsal root (directly or via the dorsal roots) stimulation of an adjacent dorsal root (not containing the recorded axon; $0.1 \mathrm{~ms}, 1-3 \mathrm{xT}$ ) evoked a characteristic large and long depolarization of the afferents, previously demonstrated to be mediated by GABAergic input onto the sensory axons ${ }^{16}$. This depolarization is termed primary afferent depolarization (PAD). PAD occurs at a minimal latency of $4-5 \mathrm{~ms}$ following the afferent volley, consistent with its trisynaptic origin ${ }^{14,16}$, making it readily distinguishable from earlier events on the axon. PAD has a fast synaptic component evoked by a single DR stimulation (rising within $30 \mathrm{~ms}$ and decaying exponentially over $<100 \mathrm{~ms}$; termed phasic PAD) and a slower longer lasting extrasynaptic component (starting at about $30 \mathrm{~ms}$ and lasting many seconds) that is enhanced by repeated DR stimulation (tonic PAD, especially with cutaneous stimulation) ${ }^{16}$. We used this sensory activation of PAD or direct optogenetic activation of PAD to examine the action of GABA on sensory axon spike transmission to motoneurons, usually evoking phasic PAD about $10-60 \mathrm{~ms}$ prior to spikes or associated EPSPs on motoneurons (during phasic PAD), though we also examined longer lasting effects of tonic PAD evoked by repeated DR stimulation. Sometimes PAD is so large that it directly evokes spikes on the afferents, and these travel out the dorsal root, and thus they have been termed dorsal root reflexes (DRRs) ${ }^{16,23}$. We usually minimized these DRRs by keeping the DR stimulus that evokes PAD low $(1.1-3.0 \mathrm{xT})$, though there were inevitably some DRRs, as they even occur in vivo in cats and humans $9,36,46$.

When we recorded from motoneurons (directly or via ventral roots) stimulation of proprioceptive afferents in a dorsal root (0.1 ms, 1.1-1.5xT) evoked a monosynaptic EPSP, and associated monosynaptic reflex (MSR, spikes from EPSP). This EPSP is depressed by fast repetition (rate depended depression, RDD) ${ }^{83}$, and thus to study the EPSP we evoked it at long intervals (10 s, $0.1 \mathrm{~Hz}$ rate) where RDD was less. However, even with this slow repetition rate $(0.1 \mathrm{~Hz})$, at the start of testing the first EPSP was often not similar to the steady state EPSP after repeated testing. Thus, to avoid RDD we usually ran the $0.1 \mathrm{~Hz}$ EPSP testing continuously throughout the experiment, at least until a steady state response was reached (after 10 minutes). We then examined the action of activating (or inhibiting) 
GABAaxo neurons on this steady state EPSP, by introducing light or sensory conditioning that activated these neurons at varying intervals (inter-stimulus intervals, ISIs) prior to each EPSP stimulation (control, GAD2//ChR2 mice and GAD2//Arch3 mice). We averaged the EPSP from $~ 10$ trials (over 100 s) just before conditioning and then 10 trials during conditioning, and then computed the change in the peak size of the monosynaptic EPSP with conditioning from these averages. After conditioning was completed EPSP testing continued and any residual changes in the EPSP were computed from the 10 trials following conditioning (after-effect). Finally, EPSP testing continued over many minutes after which the original steady state EPSP was established. The background motoneuron potential, membrane resistance $(\mathrm{Rm})$ and time constant just prior to the EPSP was also assessed before and after conditioning to examine whether there were any postsynaptic changes that might contribute to changes in the EPSP with conditioning. Along with the VR recordings, we simultaneously recorded PAD from DRs by similar averaging methods (10 trials of conditioning), to establish the relation of changes in EPSPs with associated sensory axon depolarization PAD.

\section{Drugs and solutions}

Two kinds of artificial cerebrospinal fluid (ACSF) were used in these experiments: a modified ACSF (mACSF) in the dissection chamber prior to recording and a normal ACSF (nACSF) in the recording chamber. The mACSF was composed of (in mM) $118 \mathrm{NaCl}, 24 \mathrm{NaHCO} 3,1.5 \mathrm{CaCl}, 3 \mathrm{KCl}, 5 \mathrm{MgCl}$, 1.4 NaH2PO4, 1.3 MgSO4, 25 D-glucose, and 1 kynurenic acid. Normal ACSF was composed of (in $\mathrm{mM}) 122 \mathrm{NaCl}, 24 \mathrm{NaHCO} 3,2.5 \mathrm{CaCl}, 3 \mathrm{KCl}, 1 \mathrm{MgCl}$, and $12 \mathrm{D}$-glucose. Both types of ACSF were saturated with 95\% O2-5\% CO2 and maintained at $\mathrm{pH}$ 7.4. The drugs sometimes added to the ACSF were APV (NMDA receptor antagonist), CNQX (AMPA antagonist), gabazine (GABAA antagonist), bicuculline (GABAA, antagonist), L655708 (a5 GABAA, antagonist), CGP55845 (GABAB antagonist; all from Tocris, USA), 5-HT, kynurenic acid (all from Sigma-Aldrich, USA), and TTX (TTX-citrate; Toronto Research Chemicals, Toronto). Drugs were first dissolved as a 10 - $50 \mathrm{mM}$ stock in water or DMSO before final dilution in ACSF. DMSO was necessary for dissolving gabazine, L655708, bicuculline and CGP55845, but was kept at a minimum (final DMSO concentration in ACSF $<0.04 \%$ ), which by itself had no effect on reflexes or sensory axons in vehicle controls (not shown). L655708 was particularly difficult to dissolve and precipitated easily, especially after it had been exposed a few times to air; so immediately after purchase we dissolved the entire bottle and froze it at $-40^{\circ} \mathrm{C}$ in single use 5 $20 \mu \mathrm{l}$ aliquots, and upon use it was first diluted in $100 \mu 1$ distilled water before dispersing it into ACSF.

Recording monosynaptic reflexes in awake mice and rats, and PAD activation. 
Window implant over spinal cord. In GAD2//ChR2+ mice and control GAD2//ChR- mice a glass window was implanted over the exposed spinal cord to gain optical access to the sacrocaudal spinal cord, as described previously ${ }^{65}$. Briefly, mice were given Meloxicam (1 mg/kg, s.c.) and then anesthetized using ketamine hydrochloride (100 mg/kg, i.p.) and xylazine (10 mg/kg, i.p.). Using aseptic technique, a dorsal midline incision was made over the L2 to L5 vertebrae. Approximately 0.1 $\mathrm{ml}$ of Xylocaine (1\%) was applied to the surgical area and then rinsed. The animals were suspended from a spinal-fork stereotaxic apparatus (Harvard Apparatus) and the muscles between the spinous and transverse processes were resected to expose the L2 to L5 vertebrae. The tips of modified staples were inserted along the lateral edge of the pedicles and below the lateral processes of L2 and L5, and glued in place using cyanoacrylate. A layer of cyanoacrylate was applied to all of the exposed tissue surrounding the exposed vertebrae followed by a layer of dental cement to cover the cyanoacrylate and to form a rigid ring around the exposed vertebrae. A modified paperclip was implanted in the layer of dental cement to serve as a holding point for surgery. A laminectomy was performed at L3 and L4 to expose the spinal cord caudal to the transection site. Approximately $0.1 \mathrm{ml}$ of Xylocaine (1\%) was applied directly to the spinal cord for $2-3 \mathrm{~s}$, and then rinsed. A line of Kwik-Sil (World-Precision Instruments) was applied to the dura mater surface along the midline of the spinal cord and a glass window was immediately placed over the exposed spinal cord. The window was glued in place along the outer edges using cyanoacrylate followed by a ring of dental cement. Small nuts were mounted onto this ring to later bolt on a backpack to apply the laser light (on the day of experimentation). Saline (1 ml, s.c.) and buprenorphine $(0.03 \mathrm{mg} / \mathrm{kg}$, s.c.) was administered post-operatively, and analgesia was maintained with buprenorphine $(0.03 \mathrm{mg} / \mathrm{kg}$, s.c.) every 12 hours for two days. Experimentation started 1 week after the window implant when the mouse was fully recovered.

Percutaneous EMG wire implant and fibre optic cable attachment. On the day of experimentation, the mouse was briefly sedated with isoflurane (1.5\%) and fine stainless steel wires (AS 613, Cooner Wire, Chatsworth, USA) were percutaneously implanted in the tail for recording EMG and stimulating the caudal tail trunk nerve, as we previously detailed (wires de-insulated by $2 \mathrm{~mm}$ at their tip and inserted in the core of 27 gauge needle that was removed after insertion) ${ }^{62}$. A pair of wires separated by $8 \mathrm{~mm}$ were inserted at base of the tail for recording EMG the tail muscles, and second pair of wires was inserted nearby for bipolar activation of the caudal trunk nerve to evoke reflexes. A fifth ground wire was implanted between the EMG and stimulation wires. Following this a backpack was bolted into the nuts imbedded in the dental cement ring around the window. This backpack held and aligned a light fibre optic cable that was focused on the centre of the S3 - S4 sacral spinal cord. The Cooner wires 
were secured to the skin with drops of cyanoacrylate and taped onto the backpack so that the mouse could not chew them. The isoflurane was removed, and the mouse quickly recovered from the anesthesia and was allowed to roam freely around an empty cage during recording, or was sometimes lightly restrained by hand or by a sling. The fibre optic cable was attached to a laser (447 nM, same above) and the Cooner wires attached to the same models of amplifiers and stimulators used for ex vivo monosynaptic testing detailed above.

\section{MSR testing.}

The monosynaptic reflex (MSR) was recorded in the tail EMG at $\sim 6 \mathrm{~ms}$ latency after stimulating the caudal tail trunk nerve at a low intensity that just activated proprioceptive afferents $(0.2 \mathrm{~ms}$ current pulses, $1.1 \mathrm{xT}$ ), usually near the threshold to activate motor axons and an associated M-wave (that arrived earlier). We studied the tail MSR reflex because our ex vivo recordings were made in the corresponding sacral spinal cord of adult mice and rats, which is the only portion of the spinal cord that survives whole ex vivo, due to its small diameter ${ }^{64}$. This reflex was verified to be of monosynaptic latency because it was the first reflex to arrive, had little onset jitter, and had the same latency as the F wave (not shown; the F wave is evoked by a strong stimulation of all motor axons, at 5xT, which causes a direct motoneuron response on the same axons, while the monosynaptic EPSP is blocked by collision at this intensity) ${ }^{84}$. The MSR also underwent rate dependent depression (RDD) with fast repeated stimulation and so was synaptic and not a direct muscle response (M-wave), which occurred earlier at sufficient intensity to recruit the motor axons (not shown).

Conditioning of the MSR by optogenetic activation of GABA axo neurons. As with in vitro EPSP testing, the MSR was tested repeatedly at long 5-10 s intervals until a steady state MSR was achieved. Then testing continued but with a conditioning light pulse applied just prior to the MSR stimulation (40 - 120 $\mathrm{ms}$ ), to examine the effect of PAD evoked during this time frame on sensory transmission to motoneurons. Background EMG just prior to MSR testing was assessed to estimate the postsynaptic activity on the motoneurons. The changes in MSR and background EMG with light were quantified by comparing the average response before and during the light application, computed from the mean rectified EMG at $6-11 \mathrm{~ms}$ after the nerve stimulation (MSR) and over $20 \mathrm{~ms}$ prior to the nerve stimulation (background just prior to the MSR, Bkg). Because awake mice spontaneously varied their EMG, we plotted the relation between the MSR and the background EMG, with as expected a positive linear relation between these two variables ${ }^{85}$, computed by fitting a regression line. In trials with conditioning light applied the same plot of EMG vs background EMG was made and a second 
regression line computed. The change in the MSR with conditioning at a fixed matched background EMG level was then computed for each mouse by measuring the difference between the regression line responses at a fixed background EMG. This ruled out changes in MSRs being due to postsynaptic changes. Two background levels were assessed: rest (0\%) and 30\% of maximum EMG, expressed as a percentage of the control pre-conditioning MSR. The change in background EMG with light was computed by comparing the EMG just prior to the light application (over $20 \mathrm{~ms}$ prior) to the EMG just prior to the MSR (over $20 \mathrm{~ms}$ prior, Bkg), and expressed as a percentage of the maximum EMG.

Cutaneous conditioning of the MSR in rats. A similar examination of how PAD affected the MSR was performed in rats with percutaneous tail EMG recording. However, in this case PAD was evoked by a cutaneous conditioning stimulation of the tip of the tail ( $0.2 \mathrm{~ms}$ pulses, $3 \times \mathrm{xT}, 40-120 \mathrm{~ms}$ prior to MSR testing) using an additional pair of fine Cooner wires implanted at the tip of the tail (separated by 8 $\mathrm{mm})$. In rats the MSR latency is later than in mice due to the larger peripheral conduction time, $\sim 12 \mathrm{~ms}$ (as again confirmed by a similar latency to the $\mathrm{F}$ wave). This MSR was thus quantified by averaging rectified EMG over a $12-20 \mathrm{~ms}$ window. Also, to confirm the GABAA receptor involvement in regulating the MSR, the antagonist L655708 was injected systemically ( $1 \mathrm{mg} / \mathrm{kg}$ i.p., dissolved in $50 \mu 1$ DMSO and diluted in $900 \mu \mathrm{l}$ saline). Again, the MSR was tested at matched background EMG levels before and after conditioning (or L655708 application) to rule out changes in postsynaptic inhibition.

\section{Conditioning of the MSRs in humans}

$H$-reflex as an estimate of the MSR. Participants were seated in a reclined, supine position on a padded table. The right leg was bent slightly to access the popliteal fossa and padded supports were added to facilitate complete relaxation of all leg muscles. A pair of Ag-AgCl electrodes (Kendall; Chicopee, MA, USA, $3.2 \mathrm{~cm}$ by $2.2 \mathrm{~cm}$ ) was used to record surface EMG from the soleus muscle. The EMG signals were amplified by 1000 and band-pass filtered from 10 to $1000 \mathrm{~Hz}$ (Octopus, Bortec Technologies; Calgary, AB, Canada) and then digitized at a rate of $5000 \mathrm{~Hz}$ using Axoscope 10 hardware and software (Digidata 1400 Series, Axon Instruments, Union City, CA) ${ }^{62}$. The tibial nerve was stimulated with an $\mathrm{Ag}-\mathrm{AgCl}$ electrode (Kendall; Chicopee, MA, USA, $2.2 \mathrm{~cm}$ by $2.2 \mathrm{~cm}$ ) in the popliteal fossa using a constant current stimulator (1 ms rectangular pulse, Digitimer DS7A, Hertfordshire, UK) to evoke an H-reflex in the soleus muscle, an estimate of the MSR ${ }^{37}$. Stimulation intensity was set to evoke a test (unconditioned) MSR below half maximum. MSRs recorded at rest were evoked every 5 seconds to minimize RDD ${ }^{86}$ and at least 20 test MSRs were evoked before conditioning to establish a steady baseline because the tibial nerve stimulation itself can presumably also activate spinal GABAergic 
networks, as in rats. All MSR were recorded at rest, except when the motor unit firing probabilities were measured (see below).

Conditioning of the MSR. To condition the soleus MSR by cutaneous stimulation, the cutaneous medial branch of the deep peroneal (cDP) nerve was stimulated on the dorsal surface of the ankle using a bipolar arrangement (Ag-AgCl electrodes, Kendall; Chicopee, MA, USA, $2.2 \mathrm{~cm}$ by $2.2 \mathrm{~cm}$ ), set at 1.0xT, where $\mathrm{T}$ is the threshold for cutaneous sensation. A brief burst ( 3 pulses, $200 \mathrm{~Hz}$ for $10 \mathrm{~ms}$ ) of cDP stimuli was applied before evoking a MSR at various inter-stimulus intervals (ISIs; interval between tibial and cDP nerve stimuli) within the window expected for phasic PAD evoked by cutaneous stimuli, presented in random order at 0, 30, 60, 80, 100, 150 and $200 \mathrm{~ms}$ ISIs. Seven conditioned MSR at each ISI were measured consecutively and the average of these MSR (peak-topeak) was used as an estimate of the conditioned MSR. This was compared to the average MSR without conditioning, computed from the 7 trials just prior to conditioning.

The cDP nerve was also stimulated with a $500 \mathrm{~ms}$ long train at $200 \mathrm{~Hz}$ to condition the MSR, and examine the effect of tonic PAD evoked by such long trains, as in rats. Following the application of at least 20 test MSRs (every $5 \mathrm{~s}$ ), a single cDP train was applied $700 \mathrm{~ms}$ before the next MSR and following this the MSR continued to be evoked for another 90 to $120 \mathrm{~s}$ (time frame of tonic PAD). We also conditioned the soleus MSR with tibialis anterior (TA; antagonist muscle, flexor) tendon vibration (brief burst of 3 cycles of vibration at $200 \mathrm{~Hz}$ ) to preferentially activate Ia afferents, as has been done previously ${ }^{37}$.

Motor unit recording to examine postsynaptic actions of conditioning. Surface electrodes were used to record single motor units in the soleus muscle during low level contractions by placing electrodes on or near the tendon or laterally on the border of the muscle as detailed previously ${ }^{87}$. Alternatively, single motor unit activity from the soleus muscle was also recorded using a high density surface EMG electrode (OT Bioelettronica, Torino, Italy, Semi-disposable adhesive matrix, 64 electrodes, 5x13, 8 mm inter-electrode distance) with 3 ground straps wrapped around the ankle, above and below the knee. Signals were amplified (150 times), filtered (10 to $900 \mathrm{~Hz}$ ) and digitized (16 bit at $5120 \mathrm{~Hz})$ using the Quattrocento Bioelectrical signal amplifier and OTBioLab+ v.1.2.3.0 software (OT Bioelettronica, Torino, Italy). The EMG signal was decomposed into single motor units using custom MatLab software as per ${ }^{88}$. Intramuscular EMG was used to record MUs in one participant as detailed previously ${ }^{89}$ to verify single motor unit identification from surface EMG. 
To determine if there were any postsynaptic effects from the conditioning stimulation on the motoneurons activated during the MSR, we examined whether the cDP nerve stimulation produced any changes in the tonic firing rate of single motor units, which gives a more accurate estimate of membrane potential changes in motoneurons compared to compound EMG. Single motor units were activated in the soleus muscle by the participant holding a small voluntary contraction of around $5 \%$ of maximum. Both auditory and visual feedback were used to keep the firing rates of the units steady while the conditioning cutaneous was applied every 3 to 5 seconds. The instantaneous firing frequency profiles from many stimulation trials were superimposed and time-locked to the onset of the conditioning stimulation to produce a peri-stimulus frequencygram (PSF, dots in Fig. 7biii), as previously detailed ${ }^{89,90}$. A mean firing profile resulting from the conditioning stimulation (PSF) was produced by averaging the frequency values in $20 \mathrm{~ms}$ bins across time post conditioning (thick lines in Fig. 7biii and 7ciii). To quantify if the conditioning stimulation changed the mean firing rate of the tonically firing motor units, the \% change in the mean PSF rate was computed at the time when the $\mathrm{H}$ reflex was tested (vertical line in Fig. 7bii-iii).

Unitary EPSP estimates from PSF. To more directly examine if the facilitation in MSR resulted from changes in transmission in Ia afferents after cutaneous afferent conditioning, we measured changes in the firing probability of single motor units (MUs) during the brief MSR time-course (typically 30 to 45 ms post tibial nerve stimulation) with and without cDP nerve conditioning. Soleus MSRs were as usual evoked by stimulating the tibial nerve, but while the participant held a small voluntary plantarflexion to activate tonic firing of a few single motor units. The size of the MSR was set to just above reflex threshold (and when the M-wave was $<5 \%$ of maximum) so that single motor units at the time of the MSR could be distinguished from the compound potential from many units that make up the MSR ${ }^{91}$. For a given trial run, test MSRs were evoked every 3-5 s for the first $100 \mathrm{~s}$ and then MSR testing continued for a further $100 \mathrm{~s}$, but with a cDP-conditioning train $(50 \mathrm{~ms}, 200 \mathrm{~Hz})$ applied $500 \mathrm{~ms}$ prior to each MSR testing stimulation. These repeated high frequency trains evoke a tonic PAD in rats that facilitates sensory conduction. A $500 \mathrm{~ms}$ ISI was used to ensure the firing rate of the motor unit returned to baseline before the MSR was evoked, and this is also outside of the range of phasic PAD. Approximately 40-50 usable test and conditioned firing rate profiles were produced for a single session where the motor units had a steady discharge rate before the cDP nerve stimulation. Sessions were repeated 3-6 times to obtain a sufficient number of frequency points to construct the PSF ( $\sim 200$ trials). 
To estimate the EPSP profile, motor unit (MU) firing was again used to construct a PSF, as detailed above, but this time locked to the tibial nerve stimulation used to evoke the MSR, so that we could estimate the motoneuron behaviour during the MSR (EPSP). When more than one MU was visible in the recordings firing from these units (usually $2-3$ ) were combined into a single PSF. Overall this gave about of 100 - 600 MU MSR test sweeps to generate each PSF. Firing frequency values were averaged in consecutive $20 \mathrm{~ms}$ bins to produce a mean PSF profile over time after tibial nerve stimulation, for both unconditioned and conditioned MSR reflex trials. The mean background firing rate within the 100 ms window immediately preceding the tibial stimulation was compared between the test and conditioned MSR trials to determine if the conditioning cDP nerve stimulation produced a change in firing rate, and thus post-synaptic effect, just before the conditioned MSR was evoked. For each PSF generated with or without conditioning, the probability that a motor unit discharged during the MSR window (30 to $45 \mathrm{~ms}$ after the TN stimulation) was measured as the number of discharges during the time of the MSR window divided by the total number of tibial nerve test stimuli. As an estimate of EPSP size, the mean firing rate during the MSR window was also measured (this was computed with smaller PSF bins of $0.5 \mathrm{~ms}$ during the MSR).

\section{Temperature, latency and PAD considerations.}

Large proprioceptive group Ia sensory afferents conduct in the peripheral tail nerve with a velocity of about $33 \mathrm{~m} / \mathrm{s}(33 \mathrm{~mm} / \mathrm{ms})$ in mice ${ }^{92}$. Motor axons are similar, though slightly slower $(30 \mathrm{~m} / \mathrm{s}){ }^{93}$. Thus, in the awake mouse stimulation of Ia afferents in the mouse tail evokes spikes that take $\sim 2 \mathrm{~ms}$ to conduct to the motoneurons in the spinal cord $\sim 70 \mathrm{~mm}$ away. Following $\sim 1 \mathrm{~ms}$ synaptic and spike initiation delay in motoneurons, spikes in the motor axons take a further $\sim 2 \mathrm{~ms}$ to reach the muscles, after which the EMG is generated with a further $1 \mathrm{~ms}$ synaptic and spike initiation delay at the motor endplate to produce EMG. All told this gives a monosynaptic reflex latency of $\sim 6 \mathrm{~ms}$. The motor unit potentials within the EMG signal have a duration of about $3-5 \mathrm{~ms}$, and thus we averaged rectified EMG over 6-11 ms to quantify the MSR. We have shown that similar considerations hold for the rat where tail nerve conduction velocities are similar, except the distance from the tail stimulation to the spinal cord is larger $(150 \mathrm{~mm})$, yielding a peripheral nerve conduction delay of $\sim 10 \mathrm{~ms}$ and total MSR delay of $\sim 12 \mathrm{~ms}{ }^{94}$. In humans the MSR latency is dominated by the nerve conduction latency $(50-60$ $\mathrm{m} / \mathrm{s}$ ) over a large distance $(\sim 800 \mathrm{~mm})$, yielding MSR latencies of $\sim 30 \mathrm{~ms}$.

In our ex vivo whole adult spinal cord preparation the bath temperature was varied between 23 and $32^{\circ} \mathrm{C}$. All data displayed is from $23-24^{\circ} \mathrm{C}$, though we confirmed the main results (facilitation of 
sensory axon transmission to motoneuron by PAD) at $32^{\circ} \mathrm{C}$. The Q10 for peripheral nerve conduction (ratio of conduction velocities with a $10^{\circ} \mathrm{C}$ temperature rise) is about $1.3^{95}$, yielding a conduction in dorsal roots of about $20 \mathrm{~m} / \mathrm{s}$ at $23-24^{\circ} \mathrm{C}$, as we directly confirmed (not shown). Thus, when the DR is stimulated $20 \mathrm{~mm}$ from the cord the latency of spike arrival at the cord should be about $1 \mathrm{~ms}$, which is consistent with the time of arrival of afferent volleys that were seen in the intracellular and extracellular recordings from sensory axons (e.g. Figs. $2 \mathrm{~b}$ and $4 \mathrm{e})$.

When we found that PAD evoked in sensory axons can prevent failure of spikes to propagate in the cord after DR stimulation, we worried that PAD somehow influenced the initiation of the spike by the dorsal root stimulation at the silver wire. However, we ruled this out by stimulating dorsal roots as far away from the spinal cord as possible $(20 \mathrm{~mm})$, where PAD has no effect, due to the exponential attenuation of its dorsal root potential with distance (see above), and found that PAD still facilitated sensory axon spike transmission to motoneurons. The added advantage of these long roots is that there is a clean $1 \mathrm{~ms}$ separation between the stimulus artifact and the afferent volley arriving at the spinal cord, allowing us to quantify small FPs and afferent volleys that are otherwise obscured by the artifact.

We did not consistently use high temperature ex vivo baths $\left(32^{\circ} \mathrm{C}\right)$ because the VR and DR responses to activation of DRs or PAD neurons are irreversibly reduced by prolonged periods at these temperatures, suggesting that the increased metabolic load and insufficient oxygen penetration deep in the tissue damages the cord at these temperatures. Importantly, others have reported that in sensory axons PADevoked spikes (DRRs) are eliminated in a warm bath and argued that this means they are not present in vivo, and not able to evoked a motoneuron response ${ }^{7}$, despite evidence to the contrary ${ }^{9,46}$. However, we find that PAD itself is reduced in a warm bath by the above irreversible damage, and it is thus not big enough to evoke spikes in sensory axons; thus, this does not tell us whether these spikes should be present or not in vivo. Actually, in vivo we sometimes observed that with optogenetic activation of GABA $_{\text {axo }}$ neurons and associated PAD there was a direct excitation of the motoneurons (seen in the EMG) at the latency expected for PAD evoked spikes (not shown). However, this was also at the latency of the postsynaptic inhibition produced by this same optogenetic stimulation, which often masked the excitation (Fig. 6). In retrospect, examining the GABAaxo evoked motoneuron responses during optogenetic-evoked PAD (Fink et al.) ${ }^{7,17}$, or sensory-evoked PAD ${ }^{17,18}$, there is a small excitation riding on the postsynaptic IPSPs from the activation of there GABAaxo neurons. This is consistent with the PAD evoked spike activating the monosynaptic pathway, which inhibits subsequently tested monosynaptic responses by post activation depression (see Discussion). 
The latency of a single synapse in our ex vivo preparation at $23-24^{\circ} \mathrm{C}$ was estimated from the difference between the time arrival of the sensory afferent volley at the motoneurons (terminal potential seen in intracellular and extracellular recordings) and the onset of the monosynaptic EPSP in motoneurons. This was consistently $1-1.2 \mathrm{~ms}$ (Fig. 5b and e). This is consistent with a Q10 of about $1.8-2.4$ for synaptic transmission latency ${ }^{96,97}$, and $0.4 \mathrm{~ms}$ monsynaptic latency at body temperature ${ }^{98,99}$. Based on these considerations we confirm that the PAD evoked in sensory axons is

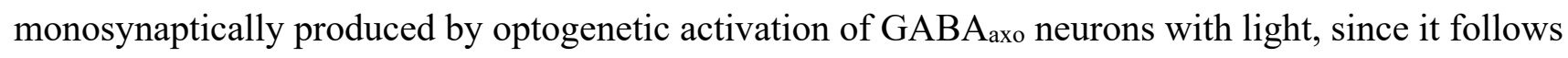
$\sim 1 \mathrm{~ms}$ after the first spike evoked in GABAaxo neurons by light (Fig. 3a). This first spike in GABAaxo neurons itself takes $1-2 \mathrm{~ms}$ to arise and so the overall latency from light activation to PAD production can be 2 - 3 ms (Fig. 3f), as seen for IPSCs at this temperature in other preparations ${ }^{100}$. With DRs stimulation PAD arises with a minimally $4-5 \mathrm{~ms}$ latency, which is consistent with a trisynaptic activation of the sensory axon, after taking into account time for spikes to arise in the interneurons involved (Fig. 4a,e).

\section{Immunohistochemisty.}

Tissue fixation and sectioning. After sensory axons in mouse and rat spinal cords were injected with neurobiotin the spinal cord was left in the recording chamber in oxygenated nACSF for an additional $4-$ $6 \mathrm{hr}$ to allow time for diffusion of the label throughout the axon. Then the spinal cord was immersed in $4 \%$ paraformaldehyde (PFA; in phosphate buffer) for $20-22$ hours at $4^{\circ} \mathrm{C}$, cryoprotected in $30 \%$ sucrose in phosphate buffer for 24-48 hours. Alternatively, afferents were labelled genetically in VGLUT1 ${ }^{\mathrm{Cre} /+}$; R26 ${ }^{\text {lsl-tdTom }}$ mice, which were euthanized with Euthanyl (BimedaMTC; $700 \mathrm{mg} / \mathrm{kg}$ ) and perfused intracardially with $10 \mathrm{ml}$ of saline for $3-4 \mathrm{~min}$, followed by $40 \mathrm{ml}$ of $4 \%$ paraformaldehyde (PFA; in $0.1 \mathrm{M}$ phosphate buffer at room temperature), over $15 \mathrm{~min}$. Then spinal cords of these mice were postfixed in PFA for $1 \mathrm{hr}$ at $4^{\circ} \mathrm{C}$, and then cryoprotected in $30 \%$ sucrose in phosphate buffer $(\sim 48 \mathrm{hrs})$. Following cryoprotection all cords were embedded in OCT (Sakura Finetek, Torrance, CA, USA), frozen at -60C with 2-methylbutane, cut on a cryostat NX70 (Fisher Scientific) in sagittal or transverse $25 \mu \mathrm{m}$ sections, and mounted on slides. Slides were frozen until further use.

Immunolabelling. The tissue sections on slides were first rinsed with phosphate buffered saline (PBS, $100 \mathrm{mM}, 10 \mathrm{~min}$ ) and then again with PBS containing 0.3\% Triton X-100 (PBS-TX, 10 min rinses used for all PBS-TX rinses). For the sodium channel antibody, we additionally incubated slides three times for 10 min each with a solution of $0.2 \%$ sodium borohydride (NaBH4, Fisher, S678-10) in PB, followed 
by a PBS rinse (4x $5 \mathrm{~min}$ ). Next, for all tissue, nonspecific binding was blocked with a $1 \mathrm{~h}$ incubation in PBS-TX with 10\% normal goat serum (NGS; S-1000, Vector Laboratories, Burlingame, USA) or normal donkey serum (NDS; ab7475, Abcam, Cambridge, UK). Sections were then incubated for at least 20 hours at room temperature with a combination of the following primary antibodies in PBS-TX with $2 \%$ NGS or NDS: rabbit anti- $\alpha_{5}$ GABAA receptor subunit (1:200; TA338505, OriGene Tech., Rockville, USA), rabbit anti- $\alpha 1$ GABAA receptor subunit (1:300; 06-868, Sigma-Aldrich, St. Louis, USA ), guinea pig anti- $\alpha 2$ GABAA receptor subunit (1:500; 224 104, Synaptic Systems, Goettingen,

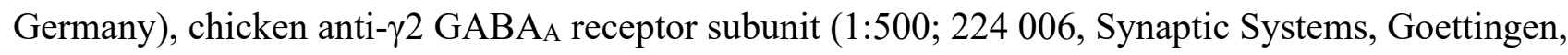
Germany), rabbit anti-GABAB1 receptor subunit (1:500; 322 102, Synaptic Systems, Goettingen, Germany), mouse anti-Neurofilament 200 (NF200) (1:2000; N0142, Sigma-Aldrich, St. Louis, USA), guinea pig anti-VGLUT1 (1:1000; AB5905, Sigma-Aldrich, St. Louis, USA), rabbit anti-Caspr (1:500; ab34151, Abcam, Cambridge, UK), mouse anti-Caspr (1:500; K65/35, NeuroMab, Davis, USA), chicken anti-Myelin Basic Protein (MBP) (1:200; ab106583, Abcam, Cambridge, UK), chicken antiVGAT (1:500; 131 006, Synaptic Systems, Goettingen, Germany), rabbit anti-VGAT (1:500; AB5062P, Sigma-Aldrich, St. Louis, USA), rabbit anti-EYFP (1:500; orb256069, Biorbyt, Riverside, UK), goat anti-RFP (1:500; orb334992, Biorbyt, Riverside, UK), rabbit anti-RFP (1:500; PM005, MBL International, Woburn, USA), rabbit anti-GFP (1:500, A11122, ThermoFisher Scientific, Waltham, USA), and mouse anti-Pan Sodium Channel (1:500; S8809, Sigma-Aldrich, St. Louis, USA). The latter is a pan-sodium antibody, labelling an intracellular peptide sequence common to all known vertebrate sodium channels. Genetically expressed EYFP, tdTom (RFP) and GFP were amplified with the above antibodies, rather than rely on the endogenous fluorescence. When anti-mouse antibodies were applied in mice tissue, the M.O.M (Mouse on Mouse) immunodetection kit was used (M.O.M; BMK-2201, Vector Laboratories, Burlingame, USA) prior to applying antibodies. This process included $1 \mathrm{~h}$ incubation with a mouse Ig blocking reagent. Primary and secondary antibody solutions were diluted in a specific M.O.M diluent.

The following day, tissue was rinsed with PBS-TX (3x $10 \mathrm{~min})$ and incubated with fluorescent secondary antibodies. The secondary antibodies used included: goat anti-rabbit Alexa Fluor 555 (1:200; A32732, ThermoFisher Scientific, Waltham, USA), goat anti-rabbit Alexa Fluor 647 (1:500, ab150079, Abcam, Cambridge, UK), goat ant-rabbit Pacific orange (1:500; P31584, ThermoFisher Scientific, Waltham, USA), goat anti-mouse Alexa Fluor 647 (1:500; A21235, ThermoFisher Scientific, Waltham, USA), goat anti-mouse Alexa Fluor 488 (1:500; A11001, ThermoFisher Scientific, Waltham, USA), goat anti-mouse Alexa Fluor 555 (1:500; A28180, ThermoFisher Scientific, Waltham, USA), goat anti- 
guinea pig Alexa Fluor 647 (1:500; A21450, ThermoFisher Scientific, Waltham, USA), goat antichicken Alexa Fluor 405 (1:200; ab175674, Abcam, Cambridge, UK), goat anti-chicken Alexa Fluor 647 (1:500; A21449, ThermoFisher Scientific, Waltham, USA), donkey anti-goat Alexa Fluor 555 (1:500; ab150130, Abcam, Cambridge, UK), donkey anti-rabbit Alexa Fluor 488 (1:500; A21206, ThermoFisher Scientific, Waltham, USA), Streptavidin-conjugated Alexa Fluor 488 (1:200; 016-540084, Jackson immunoResearch, West Grove, USA) or Streptavidin-conjugated Cyanine Cy5 (1:200; 016-170-084, Jackson immunoResearch, West Grove, USA ) in PBS-TX with 2\% NGS or NDS, applied on slides for $2 \mathrm{~h}$ at room temperature. The latter streptavidin antibodies were used to label neurobiotin filled afferents. After rinsing with PBS-TX (2 times x 10 min/each) and PBS (2 times x 10 min/each), the slides were covered with Fluoromount-G (00-4958-02, ThermoFisher Scientific, Waltham, USA) and coverslips (\#1.5, 0.175 mm, 12-544-E; Fisher Scientific, Pittsburg, USA).

Standard negative controls in which the primary antibody was either 1) omitted or 2) blocked with its antigen (quenching) were used to confirm the selectivity of the antibody staining, and no specific staining was observed in these controls. Most antibodies had been previously tested with quenching for selectivity, as detailed in the manufacture's literature and other publications ${ }^{16}$, but we verified this for the GABA receptors with quenching. For antibody quenching, the peptides used to generate the antibodies, including anti- $\alpha 5$ GABAA receptor subunit (AAP34984, Aviva Systems Biology, San Diego, USA), anti- $\alpha 1$ GABAA receptor subunit (224-2P, Synaptic Systems, Goettingen, Germany) and anti- $\gamma 2$ GABAA receptor subunit (224-1P, Synaptic Systems, Goettingen, Germany), were mixed with the antibodies at a 10:1 ratio and incubated for $20 \mathrm{~h}$ and $4^{\circ} \mathrm{C}$. This mixture was then used instead of the antibody in the above staining procedure.

\section{Confocal and epifluorescence microscopy}

Image acquisition was performed by confocal (Leica TCS SP8 Confocal System) and epifluorescence (Leica DM 6000 B) microscopy for high magnification 3D reconstruction and low magnification imaging, respectively. All the confocal images were taken with a 63x (1.4 NA) oil immersion objective lens and $0.1 \mu \mathrm{m}$ optical sections that were collected into a z-stack over 10-20 $\mu \mathrm{m}$. Excitation and recording wavelengths were set to optimize the selectivity of imaging the fluorescent secondary antibodies. The same parameters of laser intensity, gain and pinhole size was used to take pictures for each animal, including the negative controls. Complete sagittal sections were imaged with an epifluorescence 10x objective lens using the Tilescan option in Leica Application Suite X software (Leica Microsystems CMS GmbH, Germany). Sequential low power images were used to reconstruct 
the afferent extent over the whole spinal cord, using CorelDraw (Ottawa, Canada), and to identify locations where confocal images were taken.

\section{D reconstruction of afferents and localization of GABA receptors.}

The fluorescently labelled afferents (neurobiotin, tdTom), GABA receptors, VGLUT1, VGAT, NF200, Caspr, MBP and sodium channels were analyzed by 3D confocal reconstruction software in the Leica Application Suite X (Leica Microsystems CMS GmbH) ${ }^{16}$. To be very conservative in avoiding nonspecific antibody staining, a threshold was set for each fluorescence signal at a minimal level where no background staining was observed in control tissue with the primary antibody omitted, less $10 \%$.

Signals above this threshold were rendered in 3D for each antibody. Any GABA receptor, Caspr or Nav expression within the volume of the neurobiotin filled axon (binary mask set by threshold) was labelled yellow, pink and white respectively (Fig. 1), and the density within the afferents quantified using the same Leica software. Receptor densities were measured for all orders of branch sizes $\left(1^{\text {st }}, 2^{\text {nd }}, 3^{\text {rd }}\right.$ etc.; see below), for both branches dorsal to the central canal (dorsal) and ventral to the central canal (ventral). Nodes were identified with dense bands of Caspr or Na channel labelling (and lack of MBP). Branch points were also identified. We also examined raw image stacks of the neurobiotin afferents and receptors, to confirm that the automatically $3 \mathrm{D}$ reconstructed and identified receptors labelled within the afferent (yellow) corresponded to manually identified receptors colocalized with neurobiotin (Fig. 1). This was repeated for a minimum of 10 examples for each condition, and in all cases the 3D identified and manually identified receptors and channels were identical. Many receptors and channels lay outside the afferent, and near the afferent these were difficult to manually identify without the 3D reconstruction software, making the $3 \mathrm{D}$ reconstruction the only practical method to fully quantify the receptors over the entire afferent. We also optimized the reconstruction of the neurobiotin filled afferents following the methods of Fenrich ${ }^{101}$, including brightening and widening the image edges slightly (1 voxel, $0.001 \mu^{3}$ ) when necessary to join broken segments of the afferent in the final afferent reconstruction, to account for the a priori knowledge that afferents are continuous and neurobiotin signals tend to be weaker at the membrane (image edges) and in fine processes.

GABA receptors usually occurred in the axons in distinct clusters. The distances between these receptor clusters and nodes or branch points was measured and average distances computed, from high power confocal images evenly sampled across the axon arbour (employing $\sim 100$ receptor clusters from $\sim 10$ images per labelled Ia afferent). The average distance between the receptor clusters and the nearest axon terminals on the motoneurons was also computed, but this was complicated by the very large distances 
often involved, forcing us to compute the distances from low power images and relate these to the high power images of receptors sampled relatively evenly along the axon arbour. For this distance calculation, to avoid sampling bias in the high power images, we only admitted images from branch segments $\left(1^{\text {st }}, 2^{\text {nd }}\right.$ and $3^{\text {rd }}$ order, detailed below $)$ that had a receptor density within one standard deviation of the mean density in branch types with the highest density $\left(1^{\text {st }}\right.$ order ventral branches for GABAA receptors and $3^{\text {rd }}$ order ventral terminal branches for GABAB receptors; i.e. images from branches with density above the dashed confidence interval lines in Fig. 1g were included); this eliminated very large distances being included from branch segments with relatively insignificant receptor densities. We also confirmed these calculations by computing the weighted sum of all the receptor distances weighted by the sum of the receptor density for each branch type (and divided by the sum of all receptor densities), which further eliminated sampling bias. This gave similar average distance results to the above simpler analysis (not shown).

Sensory axon branch order terminology. The branches of proprioceptive axons were denoted as follows: dorsal column branches, $1^{\text {st }}$ order branches that arose of the dorsal column and project toward the motoneurons, $2^{\text {nd }}$ order branches that arose from the $1^{\text {st }}$ order branches, and $3^{\text {rd }}$ order branches that arose from the $2^{\text {nd }}$ order branches. Higher order branches occasionally arose from the $3^{\text {rd }}$ order branches, but these were collectively denoted $3^{\text {rd }}$ order branches. First and second order branches were myelinated with large dense clusters of sodium channels at the nodes in the myelin gaps, which were characteristically widely spaced. As the second order branches thinned near the transition to $3^{\text {rd }}$ order branches, they became unmyelinated, and at this point sodium channel clusters were smaller and more closely spaced ( $\sim 6 \mu \mathrm{m}$ apart, not shown). These thinned branches gave off $3^{\text {rd }}$ order (and higher) unmyelinated terminal branches with chains of characteristic terminal boutons. The $1^{\text {st }}$ order branches gave off $2^{\text {nd }}$ order branches along most of their length as they traversed the cord from the dorsal columns to the motoneurons, but we separately quantified $1^{\text {st }}, 2^{\text {nd }}$ and $3^{\text {rd }}$ order branches in the dorsal and ventral horn.

Node identification. Nodes in myelinated axon segments nodes were identified either directly via direct Na channel clusters and paranodal Caspr, or indirectly by their characteristic paranodal taper. That is, in the paranodal region the neurobiotin filled portion of the axon tapered to a smaller diameter, likely because the Caspr and presumably other proteins displaced the cytoplasmic neurobiotin, which also made the intracellular neurobiotin label less dense (Fig. 1c, black regions in taper). Regardless of the details, this taper made nodes readily identifiable. This taper forces the axial current densities to 
increase at the nodes, presumably assisting spike initiation, and consistent with previous reconstructions of myelinated proprioceptive afferents ${ }^{102}$.

\section{Computer simulations}

All computer models and simulations were implemented in NEURON ver7.5 ${ }^{103}$. The geometry and myelination pattern of the model were extracted from a previous study that used serial-section electron microscopy to generate about 15,000 photomicrographs to reconstruct a large myelinated proprioceptive Ia afferent collateral in the cat (Nicol and Walmsley, 1991). This structure was used in a prior modeling study ${ }^{34}$. Four classes of segment were defined in the model: myelinated internodes, nodes, unmyelinated bridges, and terminal boutons. Data from 18 of the 83 segments were missing from the original study. The missing data were estimated using mean values of the same segment class. The cable properties of the model were determined from diameter-dependent equations previously used for models of myelinated axons ${ }^{104}$ and included explicit representation of myelinated segments using the double cable approach ${ }^{104-106}$. Hodgkin-Huxley style models of voltage gated sodium (transient and persistent) and potassium channels were adopted from a previous study, at $37^{\circ} \mathrm{C}^{104}$. All three voltagegated conductances were colocalized to unmyelinated nodes and segments throughout the modelled axon collateral. The density of sodium and potassium conductances was adjusted to match the size and shape of experimentally recorded action potentials. To be conservative, sodium channels were placed at each node and bouton $\left(\mathrm{gNa}=1 \mathrm{~S} / \mathrm{cm}^{2}\right)$, even though bouton immunolabelling for these channels was not common in our terminal bouton imaging (Fig. 1), since disperse weak sodium channel labelling may have been missed. Removing these bouton sodium channels did not qualitatively change our computer simulation results (not shown). Current clamp stimulation was applied to the middle of the first myelinated segment (pulse width $0.1 \mathrm{~ms}$, amplitude $2 \mathrm{nA}$; near dorsal root) to initiate propagating action potentials in the model. Voltage at multiple sites of interest along the collateral was measured to assess propagation of action and graded potentials through branch points. Transient chloride conductance (i.e. GABAA receptors) was modeled using a double-exponential point process (Eq. 1); parameters were manually fit to experimental data. GABAA receptors were localized to nodes at branch points to match experimental data. The amplitude and time course of the modeled PAD (also termed PAD) was measured from the first myelinated internode segment, similar to the location of our intraaxonal recordings.

$$
\begin{aligned}
& g(t)=g_{-} \max * \beta *\left(e^{\wedge}\left((-t) / \tau_{-} \text {decay }\right)-e^{\wedge}\left((-t) / \tau_{-} \text {rise }\right)\right), \text { where } \beta=1 /\left(\left(e^{\wedge}\left((-\gamma) / \tau_{-} \text {decay }\right)-\right.\right. \\
& \left.\left.\left.e^{\wedge}\left((-\gamma) / \tau_{-} \text {rise }\right)\right)\right), \text { and } \gamma=\left(\tau_{-} \text {rise } * \tau_{-} \text {decay }\right) /\left(\tau_{-} \text {decay }-\tau_{-} \text {rise }\right) * \log \left(\tau_{-} \text {decay } / \tau_{-} \text {rise }\right) \text { (Eq. } 1\right)
\end{aligned}
$$


The parameters at all synapses were the same: time constant of rise $\left(\tau_{\text {rise }}\right)=6 \mathrm{~ms}$, time constant of decay $\left(\tau_{\text {decay }}\right)=50 \mathrm{~ms}$, default maximum conductance $\left(g_{\max }\right)=1.5 \mathrm{nS}$ (varied depending on simulation, see figure legends), and chloride reversal potential $\left(E_{\mathrm{Cl}}^{-}\right)=-25 \mathrm{mV}$ (i.e. $55 \mathrm{mV}$ positive to the resting potential to match our experimental data) ${ }^{16}$. Space constants $(\lambda s)$ were computed for each segment of the afferent, from subthreshold current injections $(100 \mathrm{~ms})$ on the distal end of each branch segment and fitting an exponential decay (with space constant $\lambda s$ ) to the passive depolarization along its length, and then repeating this with current injected in the proximal end to get a second $\lambda S$, and finally averaging these two space constants.

\section{QUANTIFICATION AND STATISTICAL ANALYSIS}

Data were analyzed in Clampfit 8.0 (Axon Instruments, USA) and Sigmaplot (Systat Software, USA). A Student's $t$-test or ANOVA (as appropriate) was used to test for statistical differences between variables, with a significance level of $P<0.05$. Power of tests was computed with $\alpha=0.05$ to design experiments. A Kolmogorov-Smirnov test for normality was applied to the data set, with a $P<0.05$ level set for significance. Most data sets were found to be normally distributed, as is required for a $t$ test. For those that were not normal a Wilcoxon Signed Rank Test was instead used with $P<0.05$. Categorical data was instead analyzed using Chi-squared tests, with Yate's continuity correction used for $2 \times 2$ contingency tables and again significant difference set at $P<0.05$. Data are plotted as bar graphs of mean \pm standard deviation (SD, error bar representing variability) or as box plots representing the interquartile range and median and error bars extend to the most extreme data point within 1.5 times the interquartile range (mean also shown as thick lines in boxes). 


\section{Data availability}

All data are available in the manuscript or the supplementary materials. Raw data are available upon request to the corresponding authors. This study did not generate data sets or new unique reagents.

\section{Code availability}

The computer code used to perform the axon simulations (Extended Date Fig. 4) are publicly available on the github repository: https://github.com/kelvinejones/noah-axon.git

\section{Acknowledgements}

We thank Leo Sanelli, Jennifer Duchcherer, Babak Afsharipour and Christopher K. Thompson for technical assistance, and Shawn Hochman, CJ Heckman, FJ Alvarez and Tia Bennett for discussions and editing the manuscript. VGLUT1 ${ }^{\mathrm{Cre}}$ mice were kindly donated by Dr. Francisco J. Alvarez. This research was supported by the Canadian Institutes of Health Research (MOP 14697 and PJT 165823 D.J.B.) and the US National Institutes of Health (NIH, R01NS47567; D.J.B. and K.F.).

\section{Author information}

Contributions. K.H, and A.M.L-O. designed the study, carried out the animal experiments and analyzed data. K.M. and M.A.G. designed and performed the human experiments. N.P. and K.E.J. designed and performed the computer simulations. S.L., S.B., A.M., M.J.S. and R.S. assisted with animal electrophysiology. K.F. and A.M.L-O provided confocal microscopy. K.K.F, S.L., D.A.R. and K.H. developed the transgenic mice and performed the optogenetic experiments. Y.L. and D.J.B. conceived and designed the study, carried out experiments and analyzed data. D.J.B, K.H., and Y.L. wrote the paper, with editing from other authors. These authors contributed equally: Krishnapriya (Veni) Hari and Ana M. Lucas-Osma. These authors jointly supervised this work as senior authors: Yaqing Li, Keith K. Fenrich and David J. Bennett.

Corresponding author. David J. Bennett

\section{Ethical declarations}

All authors declare no competing interests. 


\section{References}

1. Goulding, M. Circuits controlling vertebrate locomotion: moving in a new direction. Nature reviews. Neuroscience 10, 507-518 (2009).

2. Capaday, C. \& Stein, R.B. Amplitude modulation of the soleus H-reflex in the human during walking and standing. The Journal of neuroscience : the official journal of the Society for Neuroscience 6, 1308-1313 (1986).

3. Bennett, D.J., De Serres, S.J. \& Stein, R.B. Gain of the triceps surae stretch reflex in decerebrate and spinal cats during postural and locomotor activities. The Journal of physiology 496 ( Pt 3), 837-850 (1996).

4. Bennett, D.J. Stretch reflex responses in the human elbow joint during a voluntary movement. The Journal of physiology 474, 339-351 (1994).

5. Andrechek, E.R., et al. ErbB2 is required for muscle spindle and myoblast cell survival. Mol Cell Biol 22, 4714-4722 (2002).

6. Betley, J.N., et al. Stringent specificity in the construction of a GABAergic presynaptic inhibitory circuit. Cell 139, 161-174 (2009).

7. Fink, A.J., et al. Presynaptic inhibition of spinal sensory feedback ensures smooth movement. Nature 509, 43-48 (2014).

8. Hughes, D.I., et al. P boutons in lamina IX of the rodent spinal cord express high levels of glutamic acid decarboxylase-65 and originate from cells in deep medial dorsal horn. Proceedings of the National Academy of Sciences of the United States of America 102, 9038-9043 (2005).

9. Eccles, J.C., Eccles, R.M. \& Magni, F. Central inhibitory action attributable to presynaptic depolarization produced by muscle afferent volleys. The Journal of physiology 159, 147-166 (1961).

10. Engelman, H.S. \& MacDermott, A.B. Presynaptic ionotropic receptors and control of transmitter release. Nature reviews. Neuroscience 5, 135-145 (2004).

11. Rudomin, P. \& Schmidt, R.F. Presynaptic inhibition in the vertebrate spinal cord revisited. Experimental brain research 129, 1-37 (1999).

12. Rossignol, S., Beloozerova, I., Gossard, J.P. \& Dubuc, R. Presynaptc mechanisms during locomotion. in Presynaptic Inhibition and Neuron Control (eds. Rudmon, P., Romo, R. \& Mendell, L.M.) 385-397 (Oxford University Press, New York, 1998).

13. Ueno, M., et al. Corticospinal Circuits from the Sensory and Motor Cortices Differentially Regulate Skilled Movements through Distinct Spinal Interneurons. Cell Rep 23, 1286-1300 e1287 (2018).

14. Jankowska, E., McCrea, D., Rudomin, P. \& Sykova, E. Observations on neuronal pathways subserving primary afferent depolarization. Journal of neurophysiology 46, 506-516 (1981).

15. Zimmerman, A.L., et al. Distinct Modes of Presynaptic Inhibition of Cutaneous Afferents and Their Functions in Behavior. Neuron 102, 420-434 e428 (2019).

16. Lucas-Osma, A.M., et al. Extrasynaptic alpha5GABAA receptors on proprioceptive afferents produce a tonic depolarization that modulates sodium channel function in the rat spinal cord. Journal of neurophysiology 120, 2953-2974 (2018).

17. Fink, A.J. Exploring a behavioral role for presynaptic inhibition at spinal sensory-motor synapses. $P h D$ Thesis, Columbia University, 1-293 (2013).

18. Stuart, G.J. \& Redman, S.J. The role of GABAA and GABAB receptors in presynaptic inhibition of la EPSPS in cat spinal motoneurones. The Journal of physiology 447, 675-692 (1992).

19. Bardoni, R., et al. Pre- and postsynaptic inhibitory control in the spinal cord dorsal horn. Annals of the New York Academy of Sciences 1279, 90-96 (2013).

20. Szabadics, J., et al. Excitatory effect of GABAergic axo-axonic cells in cortical microcircuits. Science 311, 233-235 (2006).

21. Howell, R.D. \& Pugh, J.R. Biphasic modulation of parallel fibre synaptic transmission by co-activation of presynaptic GABAA and GABAB receptors in mice. The Journal of physiology 594, 3651-3666 (2016). 
22. Trigo, F.F., Marty, A. \& Stell, B.M. Axonal GABAA receptors. The European journal of neuroscience 28, 841-848 (2008).

23. Barron, D.H. \& Matthews, B.H. The interpretation of potential changes in the spinal cord. The Journal of physiology 92, 276-321 (1938).

24. Cattaert, D. \& El Manira, A. Shunting versus inactivation: analysis of presynaptic inhibitory mechanisms in primary afferents of the crayfish. The Journal of neuroscience : the official journal of the Society for Neuroscience 19, 6079-6089 (1999).

25. Debanne, D., Campanac, E., Bialowas, A., Carlier, E. \& Alcaraz, G. Axon physiology. Physiol Rev 91, 555602 (2011).

26. Goldstein, S.S. \& Rall, W. Changes of action potential shape and velocity for changing core conductor geometry. Biophysical journal 14, 731-757 (1974).

27. Burke, R.E. \& Glenn, L.L. Horseradish peroxidase study of the spatial and electrotonic distribution of group la synapses on type-identified ankle extensor motoneurons in the cat. The Journal of comparative neurology 372, 465-485 (1996).

28. Henneman, E., Luscher, H.R. \& Mathis, J. Simultaneously active and inactive synapses of single la fibres on cat spinal motoneurones. The Journal of physiology 352, 147-161 (1984).

29. Luscher, H.R., Ruenzel, P. \& Henneman, E. Composite EPSPs in motoneurons of different sizes before and during PTP: implications for transmission failure and its relief in la projections. Journal of neurophysiology 49, 269-289 (1983).

30. Wall, P.D. \& McMahon, S.B. Long range afferents in rat spinal cord. III. Failure of impulse transmission in axons and relief of the failure after rhizotomy of dorsal roots. Philosophical transactions of the Royal Society of London. Series B, Biological sciences 343, 211-223 (1994).

31. Wall, P.D. Excitability changes in afferent fibre terminations and their relation to slow potentials. The Journal of physiology 142, i3-21 (1958).

32. Chua, H.C. \& Chebib, M. GABAA Receptors and the Diversity in their Structure and Pharmacology. Adv Pharmacol 79, 1-34 (2017).

33. Lucas-Osma, A.M., et al. 5-HT1D receptors inhibit the monosynaptic stretch reflex by modulating C-fiber activity. Journal of neurophysiology 121, 1591-1608 (2019).

34. Walmsley, B., Graham, B. \& Nicol, M.J. Serial E-M and simulation study of presynaptic inhibition along a group la collateral in the spinal cord. Journal of neurophysiology 74, 616-623 (1995).

35. Redman, S. Quantal analysis of synaptic potentials in neurons of the central nervous system. Physiol Rev 70, 165-198 (1990).

36. Shefner, J.M., Buchthal, F. \& Krarup, C. Recurrent potentials in human peripheral sensory nerve: possible evidence of primary afferent depolarization of the spinal cord. Muscle Nerve 15, 1354-1363 (1992).

37. Hultborn, H., Meunier, S., Morin, C. \& Pierrot-Deseilligny, E. Assessing changes in presynaptic inhibition of I a fibres: a study in man and the cat. The Journal of physiology 389, 729-756 (1987).

38. Zbili, M. \& Debanne, D. Past and Future of Analog-Digital Modulation of Synaptic Transmission. Frontiers in cellular neuroscience 13, 160 (2019).

39. Zorrilla de San Martin, J., Trigo, F.F. \& Kawaguchi, S.Y. Axonal GABAA receptors depolarize presynaptic terminals and facilitate transmitter release in cerebellar Purkinje cells. The Journal of physiology 595, 7477-7493 (2017).

40. Burke, K.J., Jr. \& Bender, K.J. Modulation of Ion Channels in the Axon: Mechanisms and Function. Frontiers in cellular neuroscience 13, 221 (2019).

41. Rudomin, P., Nunez, R., Madrid, J. \& Burke, R.E. Primary afferent hyperpolarization and presynaptic facilitation of la afferent terminals induced by large cutaneous fibers. Journal of neurophysiology 37, 413-429 (1974).

42. Aimonetti, J.M., Vedel, J.P., Schmied, A. \& Pagni, S. Mechanical cutaneous stimulation alters la presynaptic inhibition in human wrist extensor muscles: a single motor unit study. The Journal of physiology 522 Pt 1, 137-145 (2000). 
43. Curtis, D.R. \& Lacey, G. GABA-B receptor-mediated spinal inhibition. Neuroreport 5, 540-542 (1994).

44. Pierce, J.P. \& Mendell, L.M. Quantitative ultrastructure of la boutons in the ventral horn: scaling and positional relationships. The Journal of neuroscience : the official journal of the Society for Neuroscience 13, 4748-4763 (1993).

45. McCrea, D.A., Shefchyk, S.J. \& Carlen, P.L. Large reductions in composite monosynaptic EPSP amplitude following conditioning stimulation are not accounted for by increased postsynaptic conductances in motoneurons. Neuroscience letters 109, 117-122 (1990).

46. Beloozerova, I. \& Rossignol, S. Antidromic discharges in dorsal roots of decerebrate cats. I. Studies at rest and during fictive locomotion. Brain research 846, 87-105 (1999).

47. Bos, R., Brocard, F. \& Vinay, L. Primary afferent terminals acting as excitatory interneurons contribute to spontaneous motor activities in the immature spinal cord. The Journal of neuroscience : the official journal of the Society for Neuroscience 31, 10184-10188 (2011).

48. Russ, J.B., Verina, T., Comer, J.D., Comi, A.M. \& Kaltschmidt, J.A. Corticospinal tract insult alters GABAergic circuitry in the mammalian spinal cord. Front Neural Circuits 7, 150 (2013).

49. Nelson, S.G., Collatos, T.C., Niechaj, A. \& Mendell, L.M. Immediate increase in la-motoneuron synaptic transmission caudal to spinal cord transection. Journal of neurophysiology 42, 655-664 (1979).

50. Zehr, E.P. \& Stein, R.B. Interaction of the Jendrassik maneuver with segmental presynaptic inhibition. Experimental brain research 124, 474-480 (1999).

51. Pinol, R.A., Bateman, R. \& Mendelowitz, D. Optogenetic approaches to characterize the long-range synaptic pathways from the hypothalamus to brain stem autonomic nuclei. Journal of neuroscience methods 210, 238-246 (2012).

52. Zhang, F., et al. The microbial opsin family of optogenetic tools. Cell 147, 1446-1457 (2011).

53. Chow, B.Y., et al. High-performance genetically targetable optical neural silencing by light-driven proton pumps. Nature 463, 98-102 (2010).

54. Kralj, J.M., Douglass, A.D., Hochbaum, D.R., Maclaurin, D. \& Cohen, A.E. Optical recording of action potentials in mammalian neurons using a microbial rhodopsin. Nature methods 9, 90-95 (2011).

55. Taniguchi, H., et al. A resource of Cre driver lines for genetic targeting of GABAergic neurons in cerebral cortex. Neuron 71, 995-1013 (2011).

56. Madisen, L., et al. A toolbox of Cre-dependent optogenetic transgenic mice for light-induced activation and silencing. Nat Neurosci 15, 793-802 (2012).

57. Madisen, L., et al. A robust and high-throughput Cre reporting and characterization system for the whole mouse brain. Nat Neurosci 13, 133-140 (2010).

58. Harris, J.A., et al. Anatomical characterization of Cre driver mice for neural circuit mapping and manipulation. Front Neural Circuits 8, 76 (2014).

59. Todd, A.J., et al. The expression of vesicular glutamate transporters VGLUT1 and VGLUT2 in neurochemically defined axonal populations in the rat spinal cord with emphasis on the dorsal horn. The European journal of neuroscience 17, 13-27 (2003).

60. Feil, R., Wagner, J., Metzger, D. \& Chambon, P. Regulation of Cre recombinase activity by mutated estrogen receptor ligand-binding domains. Biochem Biophys Res Commun 237, 752-757 (1997).

61. Harvey, P.J., Li, Y., Li, X. \& Bennett, D.J. Persistent sodium currents and repetitive firing in motoneurons of the sacrocaudal spinal cord of adult rats. Journal of neurophysiology 96, 1141-1157 (2006).

62. Murray, K.C., et al. Recovery of motoneuron and locomotor function after spinal cord injury depends on constitutive activity in 5-HT2C receptors. Nature medicine 16, 694-700 (2010).

63. Murray, K.C., et al. Polysynaptic excitatory postsynaptic potentials that trigger spasms after spinal cord injury in rats are inhibited by 5-HT1B and 5-HT1F receptors. Journal of neurophysiology 106, 925-943 (2011).

64. Li, Y., Gorassini, M.A. \& Bennett, D.J. Role of persistent sodium and calcium currents in motoneuron firing and spasticity in chronic spinal rats. Journal of neurophysiology 91, 767-783 (2004).

65. Lin, S., et al. Locomotor-related V3 interneurons initiate and coordinate muscles spasms after spinal cord injury. Journal of neurophysiology 121, 1352-1367 (2019). 
66. El-Gaby, M., et al. Archaerhodopsin Selectively and Reversibly Silences Synaptic Transmission through Altered pH. Cell Rep 16, 2259-2268 (2016).

67. Li, Y., et al. Pericytes impair capillary blood flow and motor function after chronic spinal cord injury. Nature medicine 23, 733-741 (2017).

68. Li, Y., Harvey, P.J., Li, X. \& Bennett, D.J. Spastic long-lasting reflexes of the chronic spinal rat studied in vitro. Journal of neurophysiology 91, 2236-2246 (2004).

69. Luscher, C., Streit, J., Lipp, P. \& Luscher, H.R. Action potential propagation through embryonic dorsal root ganglion cells in culture. II. Decrease of conduction reliability during repetitive stimulation. Journal of neurophysiology 72, 634-643 (1994).

70. Wall, P.D. Some unanswered questions about the mechanims and function of presynaptic inhibition. in Presynaptic Inhibition and Neuron Control (eds. Rudmon, P., Romo, R. \& Mendell, L.M.) 228-241 (Oxford University Press, New York, 1998).

71. Hubbard, J.I., Llinas, R. \& Quastel, D.M.J. Electrophysiological Analysis of Synaptic Transmission, (Edward Arnold Ltd, London, 1969).

72. Munson, J.B. \& Sypert, G.W. Properties of single central la afferent fibres projecting to motoneurones. The Journal of physiology 296, 315-327 (1979).

73. Dudel, J. The Mechanism of Presynaptic Inhibition at the Crayfish Neuromuscular Junction. Pflugers Arch Gesamte Physiol Menschen Tiere 284, 66-80 (1965).

74. Katz, B. \& Miledi, R. Propagation of Electric Activity in Motor Nerve Terminals. Proc R Soc Lond B Biol Sci 161, 453-482 (1965).

75. Armstrong, C.M. \& Cota, G. Calcium ion as a cofactor in Na channel gating. Proceedings of the National Academy of Sciences of the United States of America 88, 6528-6531 (1991).

76. Stein, R.B. Nerve and Muscle : Membranes, Cells, and Systems, (Springer US, Boston, MA, 1980).

77. Luscher, H.R., Ruenzel, P., Fetz, E. \& Henneman, E. Postsynatpic population potentials recorded from ventral roots perfused with isotonic sucrose: connections of groups la and II spindle afferent fibers with large populations of motoneurons. Journal of neurophysiology 42, 1146-1164 (1979).

78. Leppanen, L. \& Stys, P.K. Ion transport and membrane potential in CNS myelinated axons I. Normoxic conditions. Journal of neurophysiology 78, 2086-2094 (1997).

79. Rushton, W.A. A theory of the effects of fibre size in medullated nerve. The Journal of physiology 115, 101-122 (1951).

80. Arbuthnott, E.R., Boyd, I.A. \& Kalu, K.U. Ultrastructural dimensions of myelinated peripheral nerve fibres in the cat and their relation to conduction velocity. The Journal of physiology 308, 125-157 (1980).

81. Blight, A.R. Computer simulation of action potentials and afterpotentials in mammalian myelinated axons: the case for a lower resistance myelin sheath. Neuroscience 15, 13-31 (1985).

82. Fedirchuk, B., et al. Spontaneous network activity transiently depresses synaptic transmission in the embryonic chick spinal cord. The Journal of neuroscience : the official journal of the Society for Neuroscience 19, 2102-2112 (1999).

83. Boulenguez, P., et al. Down-regulation of the potassium-chloride cotransporter KCC2 contributes to spasticity after spinal cord injury. Nature medicine 16, 302-307 (2010).

84. Stalberg, E., et al. Standards for quantification of EMG and neurography. Clin Neurophysiol 130, 16881729 (2019).

85. Matthews, P.B. Observations on the automatic compensation of reflex gain on varying the pre-existing level of motor discharge in man. The Journal of physiology 374, 73-90 (1986).

86. Hultborn, H., et al. On the mechanism of the post-activation depression of the H-reflex in human subjects. Experimental brain research 108, 450-462 (1996).

87. Matthews, P.B. Relationship of firing intervals of human motor units to the trajectory of post-spike after-hyperpolarization and synaptic noise. The Journal of physiology 492 ( Pt 2), 597-628 (1996).

88. Negro, F., Muceli, S., Castronovo, A.M., Holobar, A. \& Farina, D. Multi-channel intramuscular and surface EMG decomposition by convolutive blind source separation. J Neural Eng 13, 026027 (2016). 
89. Norton, J.A., Bennett, D.J., Knash, M.E., Murray, K.C. \& Gorassini, M.A. Changes in sensory-evoked synaptic activation of motoneurons after spinal cord injury in man. Brain 131, 1478-1491 (2008).

90. Turker, K.S. \& Powers, R.K. Black box revisited: a technique for estimating postsynaptic potentials in neurons. Trends Neurosci 28, 379-386 (2005).

91. Nielsen, J.B., et al. Recruitment gain of spinal motor neuron pools in cat and human. Experimental brain research 237, 2897-2909 (2019).

92. Walsh, M.E., et al. Use of Nerve Conduction Velocity to Assess Peripheral Nerve Health in Aging Mice. J Gerontol A Biol Sci Med Sci 70, 1312-1319 (2015).

93. Rasminsky, M., Kearney, R.E., Aguayo, A.J. \& Bray, G.M. Conduction of nervous impulses in spinal roots and peripheral nerves of dystrophic mice. Brain research 143, 71-85 (1978).

94. Bennett, D.J., Sanelli, L., Cooke, C.L., Harvey, P.J. \& Gorassini, M.A. Spastic long-lasting reflexes in the awake rat after sacral spinal cord injury. Journal of neurophysiology 91, 2247-2258 (2004).

95. Leandri, M., Leandri, S. \& Lunardi, G. Effect of temperature on sensory and motor conduction of the rat tail nerves. Neurophysiol Clin 38, 297-304 (2008).

96. Czeh, G. \& Dezso, G.T. Separation of temperature sensitive and temperature insensitive components of the postsynaptic potentials in the frog motoneurons. Neuroscience 7, 2105-2115 (1982).

97. Silver, R.A., Cull-Candy, S.G. \& Takahashi, T. Non-NMDA glutamate receptor occupancy and open probability at a rat cerebellar synapse with single and multiple release sites. The Journal of physiology 494 ( Pt 1), 231-250 (1996).

98. Lev-Tov, A., Fleshman, J.W. \& Burke, R.E. Primary afferent depolarization and presynaptic inhibition of monosynaptic group la EPSPs during posttetanic potentiation. Journal of neurophysiology 50, 413-427 (1983).

99. Munson, J.B. \& Sypert, G.W. Properties of single fibre excitatory post-synaptic potentials in triceps surae motoneurones. The Journal of physiology 296, 329-342 (1979).

100. Takahashi, T. The minimal inhibitory synaptic currents evoked in neonatal rat motoneurones. The Journal of physiology 450, 593-611 (1992).

101. Fenrich, K.K., Zhao, E.Y., Wei, Y., Garg, A. \& Rose, P.K. Isolating specific cell and tissue compartments from 3D images for quantitative regional distribution analysis using novel computer algorithms. Journal of neuroscience methods 226, 42-56 (2014).

102. Nicol, M.J. \& Walmsley, B. A serial section electron microscope study of an identified la afferent collateral in the cat spinal cord. The Journal of comparative neurology 314, 257-277 (1991).

103. Hines, M.L. \& Carnevale, N.T. NEURON: a tool for neuroscientists. Neuroscientist 7, 123-135 (2001).

104. McIntyre, C.C., Richardson, A.G. \& Grill, W.M. Modeling the excitability of mammalian nerve fibers: influence of afterpotentials on the recovery cycle. Journal of neurophysiology 87, 995-1006 (2002).

105. Cohen, C.C.H., et al. Saltatory Conduction along Myelinated Axons Involves a Periaxonal Nanocircuit. Cell 180, 311-322 e315 (2020).

106. Stephanova, D.I. \& Bostock, H. A distributed-parameter model of the myelinated human motor nerve fibre: temporal and spatial distributions of action potentials and ionic currents. Biol Cybern 73, 275-280 (1995).

107. Boyden, E.S., Zhang, F., Bamberg, E., Nagel, G. \& Deisseroth, K. Millisecond-timescale, genetically targeted optical control of neural activity. Nat Neurosci 8, 1263-1268 (2005).

108. Olsen, R.W. \& Sieghart, W. GABA A receptors: subtypes provide diversity of function and pharmacology. Neuropharmacology 56, 141-148 (2009).

109. Chuang, S.H. \& Reddy, D.S. Genetic and Molecular Regulation of Extrasynaptic GABA-A Receptors in the Brain: Therapeutic Insights for Epilepsy. J Pharmacol Exp Ther 364, 180-197 (2018).

110. Lagrange, A.H., Hu, N. \& Macdonald, R.L. GABA beyond the synapse: defining the subtype-specific pharmacodynamics of non-synaptic GABAA receptors. The Journal of physiology 596, 4475-4495 (2018).

111. Persohn, E., Malherbe, P. \& Richards, J.G. In situ hybridization histochemistry reveals a diversity of GABAA receptor subunit $m R N A s$ in neurons of the rat spinal cord and dorsal root ganglia. Neuroscience 42, 497-507 (1991). 
112. Paul, J., Zeilhofer, H.U. \& Fritschy, J.M. Selective distribution of GABA(A) receptor subtypes in mouse spinal dorsal horn neurons and primary afferents. The Journal of comparative neurology 520, 3895-3911 (2012).

113. Rudomin, P. Presynaptic selection of afferent inflow in the spinal cord. Journal of physiology, Paris 93, 329-347 (1999).

114. Prochazka, A. \& Gorassini, M. Ensemble firing of muscle afferents recorded during normal locomotion in cats. The Journal of physiology 507 ( Pt 1), 293-304 (1998).

115. Eccles, J.C., Magni, F. \& Willis, W.D. Depolarization of central terminals of Group I afferent fibres from muscle. The Journal of physiology 160, 62-93 (1962).

116. Sypert, G.W., Munson, J.B. \& Fleshman, J.W. Effect of presynaptic inhibition on axonal potentials, terminal potentials, focal synaptic potentials, and EPSPs in cat spinal cord. Journal of neurophysiology 44, 792-803 (1980).

117. Duchen, M.R. Excitation of mouse motoneurones by GABA-mediated primary afferent depolarization. Brain research 379, 182-187 (1986).

118. Curtis, D.R. Two types of inhibition in the spinal cord. . in Presynaptic Inhibition and Neuron Control (eds. Rudmon, P., Romo, R. \& Mendell, L.M.) 150-161 (Oxford University Press, New York, 1998).

119. Redman, S.J. The relative contributions of GABAA and GABAB receptors to presynaptic inhibition of group la EPSPs. in Presynaptic Inhibition and Neuron Control (eds. Rudmon, P., Romo, R. \& Mendell, L.M.) 162-177 (Oxford University Press, New York, 1998).

120. Lloyd, D.P. Post-tetanic potentiation of response in monosynaptic reflex pathways of the spinal cord. The Journal of general physiology 33, 147-170 (1949).

121. Bostock, H. \& Grafe, P. Activity-dependent excitability changes in normal and demyelinated rat spinal root axons. The Journal of physiology 365, 239-257 (1985).

122. Lomeli, J., Quevedo, J., Linares, P. \& Rudomin, P. Local control of information flow in segmental and ascending collaterals of single afferents. Nature 395, 600-604 (1998).

123. Frank, K. Basic mechanisms of synaptic transmission in the central nervous system. Inst. Radio Eng. Trans. Med. Electron. ME-6, 85-88 (1959).

124. Frank, K. \& Fortes, M.G.F. Presynaptic and postsynaptic inhibition of monosynaptic reflexes. . Fed Proc 16, 39-40 (1957).

125. Eccles, J.C. \& Krnjevic, K. Potential changes recorded inside primary afferent fibres within the spinal cord. The Journal of physiology 149, 250-273 (1959).

126. Lev-Tov, A., Meyers, D.E. \& Burke, R.E. Activation of type B gamma-aminobutyric acid receptors in the intact mammalian spinal cord mimics the effects of reduced presynaptic $\mathrm{Ca} 2+$ influx. Proceedings of the National Academy of Sciences of the United States of America 85, 5330-5334 (1988).

127. Hultborn, H., et al. On the mechanism of the post-activation depression of the H-reflex in human subjects. Experimental brain research 108, 450-462 (1996).

128. Eccles, J.C., Schmidt, R. \& Willis, W.D. Pharmacological Studies on Presynaptic Inhibition. The Journal of physiology 168, 500-530 (1963).

129. Graham, B. \& Redman, S. A simulation of action potentials in synaptic boutons during presynaptic inhibition. Journal of neurophysiology 71, 538-549 (1994).

130. Segev, I. Computer study of presynaptic inhibition controlling the spread of action potentials into axonal terminals. Journal of neurophysiology 63, 987-998 (1990).

131. Verdier, D., Lund, J.P. \& Kolta, A. Synaptic inputs to trigeminal primary afferent neurons cause firing and modulate intrinsic oscillatory activity. Journal of neurophysiology 92, 2444-2455 (2004).

132. Luscher, C., Streit, J., Quadroni, R. \& Luscher, H.R. Action potential propagation through embryonic dorsal root ganglion cells in culture. I. Influence of the cell morphology on propagation properties. Journal of neurophysiology 72, 622-633 (1994).

133. Barron, D.H. \& Matthews, B.H. Intermittent conduction in the spinal cord. The Journal of physiology 85, 73-103 (1935). 
134. Li, Y., et al. Branching points of primary afferent fibers are vital for the modulation of fiber excitability by epidural DC polarization and by GABA in the rat spinal cord. Journal of neurophysiology 124, 49-62 (2020).

135. Gemes, G., et al. Failure of action potential propagation in sensory neurons: mechanisms and loss of afferent filtering in C-type units after painful nerve injury. The Journal of physiology 591, 1111-1131 (2013).

136. Howland, B., Lettvin, J.Y., McCulloch, W.S., Pitts, W. \& Wall, P.D. Reflex inhibition by dorsal root interaction. Journal of neurophysiology 18, 1-17 (1955).

137. Alvarez, F.J., Taylor-Blake, B., Fyffe, R.E., De Blas, A.L. \& Light, A.R. Distribution of immunoreactivity for the beta 2 and beta 3 subunits of the GABAA receptor in the mammalian spinal cord. The Journal of comparative neurology 365, 392-412 (1996).

138. Salio, C., Merighi, A. \& Bardoni, R. GABAB receptors-mediated tonic inhibition of glutamate release from Abeta fibers in rat laminae III/IV of the spinal cord dorsal horn. Molecular pain 13, 1744806917710041 (2017). 
a

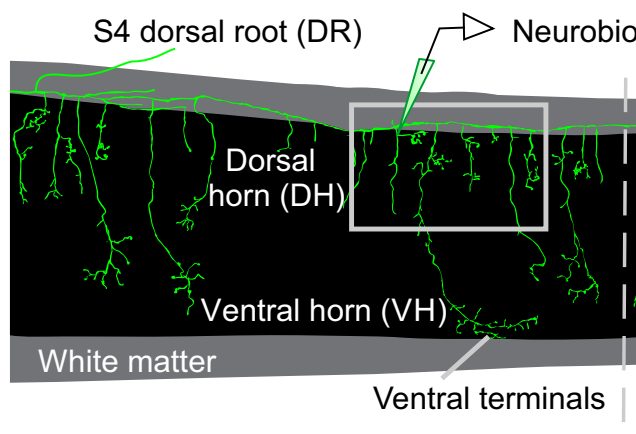

Sagittal view of sacral S4 spinal cord $\longleftarrow$
Caudal Ca1 spinal cord b

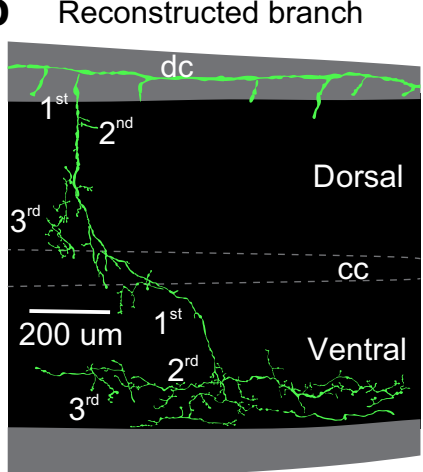

C

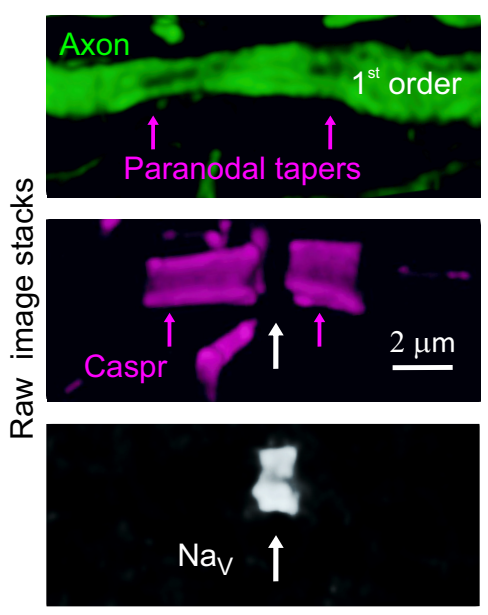

e Dorsal nodes at branch points (bp)

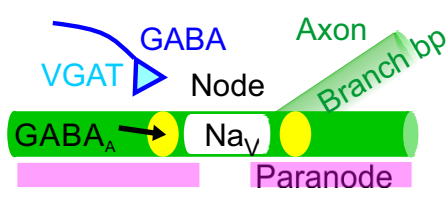

f

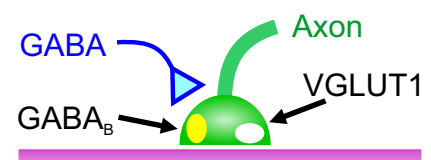

Motoneuron (mn, NF200)

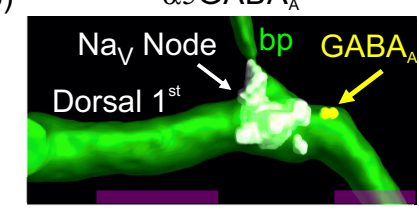

$\alpha 5 \mathrm{GABA}_{\mathrm{A}}$

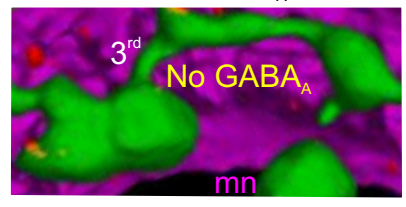

d $\mathrm{GABA}_{\mathrm{A}}$ receptors at nodes and branch points (bp)
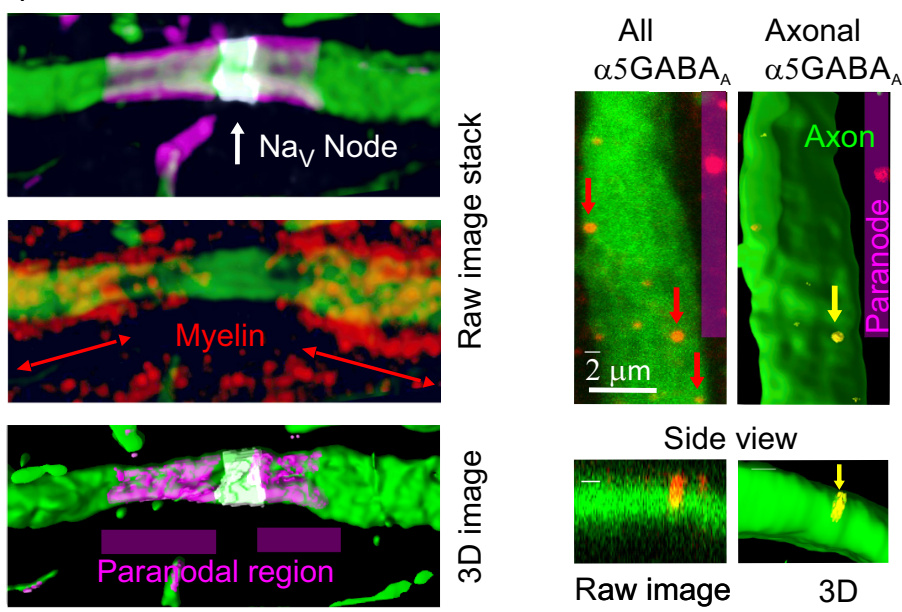

Raw image
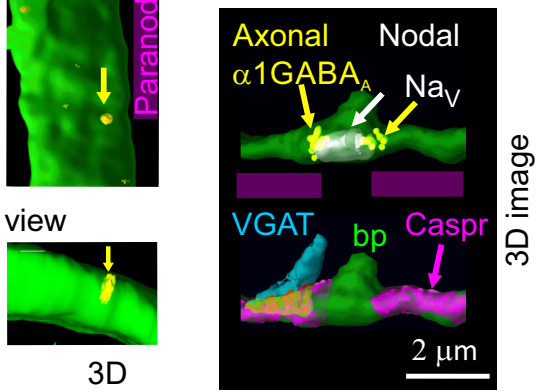

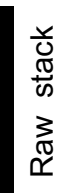

$\alpha 1 \mathrm{GABA}_{\mathrm{A}}$

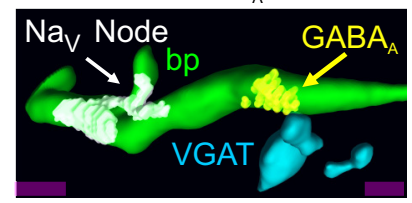

$\alpha 1 \mathrm{GABA}_{\mathrm{A}}$

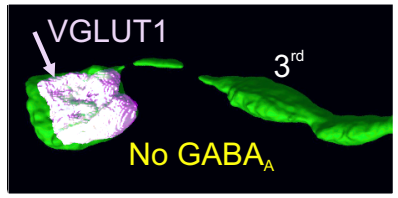

$\mathrm{GABA}_{\mathrm{B}}$

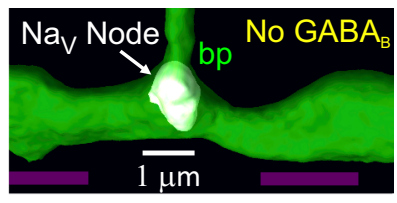

$\mathrm{GABA}_{\mathrm{B}}$

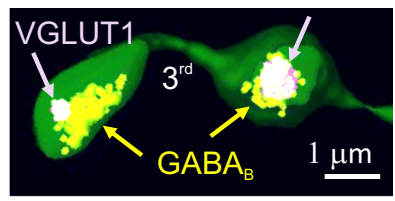

g
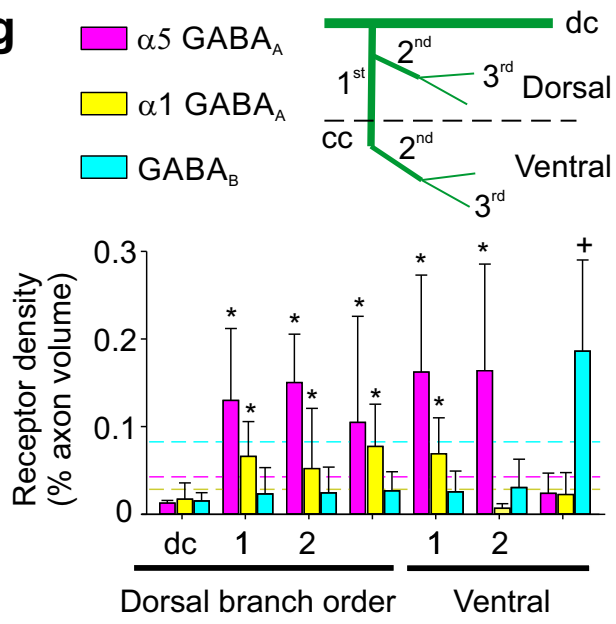

h
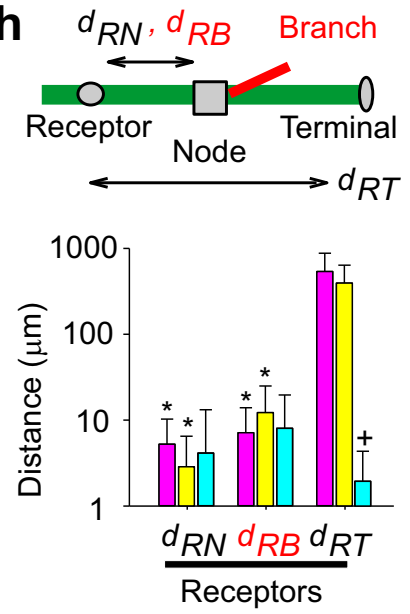
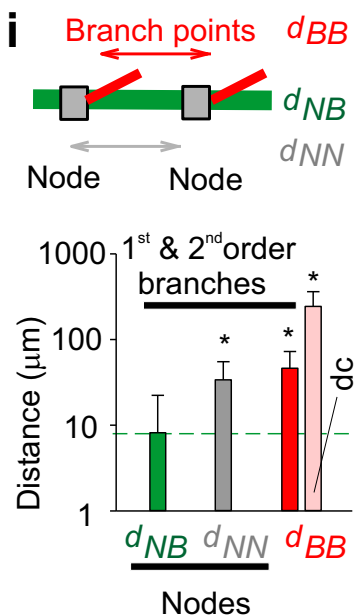

j
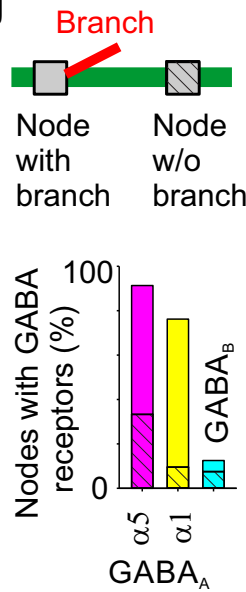
Fig. 1 | Nodal GABA $\mathbf{A}_{\mathbf{A}}$ and terminal GABA $_{\mathbf{B}}$ receptors in rats. a,b, Neurobiotin filled proprioceptive Ia axon in the sacrocaudal rat spinal cord (S4 and Ca1), reconstructed from fluorescent images (inset), with branching order indicated. Some ventral branches truncated in (a), but full arbour shown in (b). Central canal: cc. Dorsal columns: dc. Dorsal and ventral horns: DH and VH. c, Node on axon branch in DH immunolabelled for sodium channels (Nav), paranodal Caspr and myelin (MBP), with the paranodal taper indicated, and co-labelling within the axon rendered in $3 \mathrm{D}$ (bottom). $1^{\text {st }}$ order branch in DH. d-f, $\alpha 5$ GABAA, $\alpha 1$ GABAA and GABAB receptor immunolabelling (yellow) in 3D reconstructed axon branches in DH (also raw images in $\mathbf{d}$, for $\alpha 5$ and $\alpha 1$ ), with nodes identified by Nav and paranodal taper, GABA contacts by vesicular inhibitory amino acid transporter (VGAT, e) labelling, and terminals by vesicular glutamate transporter 1 (VGLUT1, f) or nearby NF200 (on motoneurons in VH). $1^{\text {st }}$ to $2^{\text {nd }}$ order branch points (bp) in DH. g, Receptor densities on axon branches of varying order. Mean $\pm S D$ plotted. Dashed lines: lower confidence interval, 1 SD below mean maximum density. *significantly more than ventral terminal ( $3^{\text {rd }}$ order) receptor density, + ventral terminal receptor density significantly more than $1^{\text {st }}$ and $2^{\text {nd }}$ order branch densities, $P<0.05, n=10-17$ branches per condition. $\mathbf{h}$, Distances between GABA receptor clusters and nodes $\left(\mathrm{d}_{\mathrm{RN}}\right)$, branch points $\left(\mathrm{d}_{\mathrm{RB}}\right)$ or terminals $\left(\mathrm{d}_{\mathrm{RT}}\right)$. * significantly less than $\mathrm{d}_{\mathrm{RT}}$. + significantly less than $\mathrm{d}_{\mathrm{RN}}$ and $\mathrm{d}_{\mathrm{RB}}, P<0.05, n=80-110$ clusters per condition. $\mathbf{i}$, Distances between branch points $\left(\mathrm{d}_{\mathrm{BB}}\right)$, nodes (Nav clusters, $\left.\mathrm{d}_{\mathrm{NN}}\right)$, and nearest branch to node $\left(\mathrm{d}_{\mathrm{NB}}\right)$.

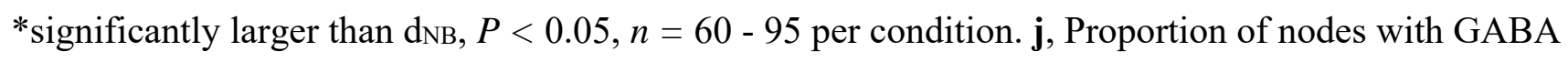
receptors, with and without (hashed) nearby branch points. 
a Ex vivo whole spinal cord
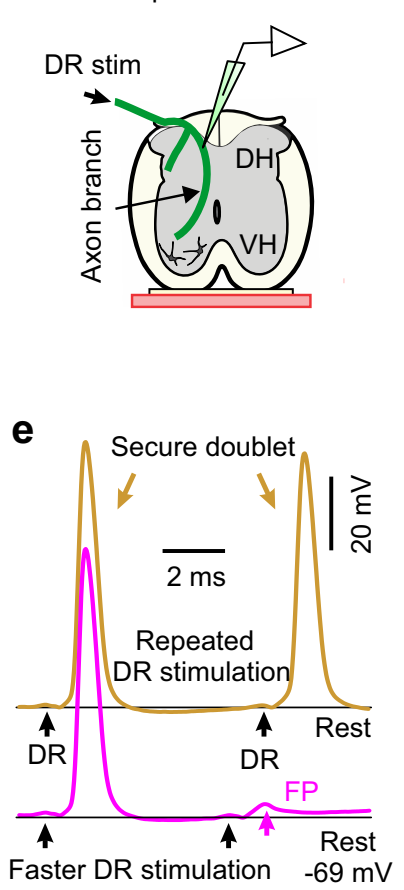
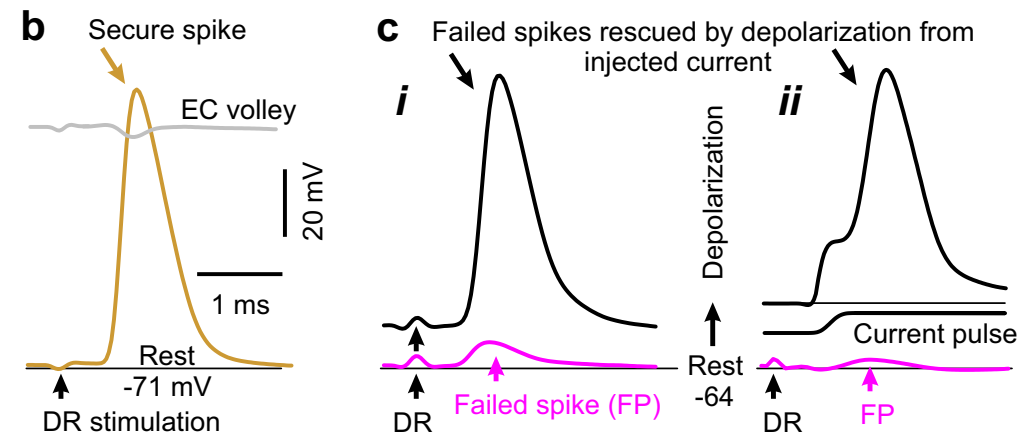

d Failed spike rescued by spontaneous depolariz.
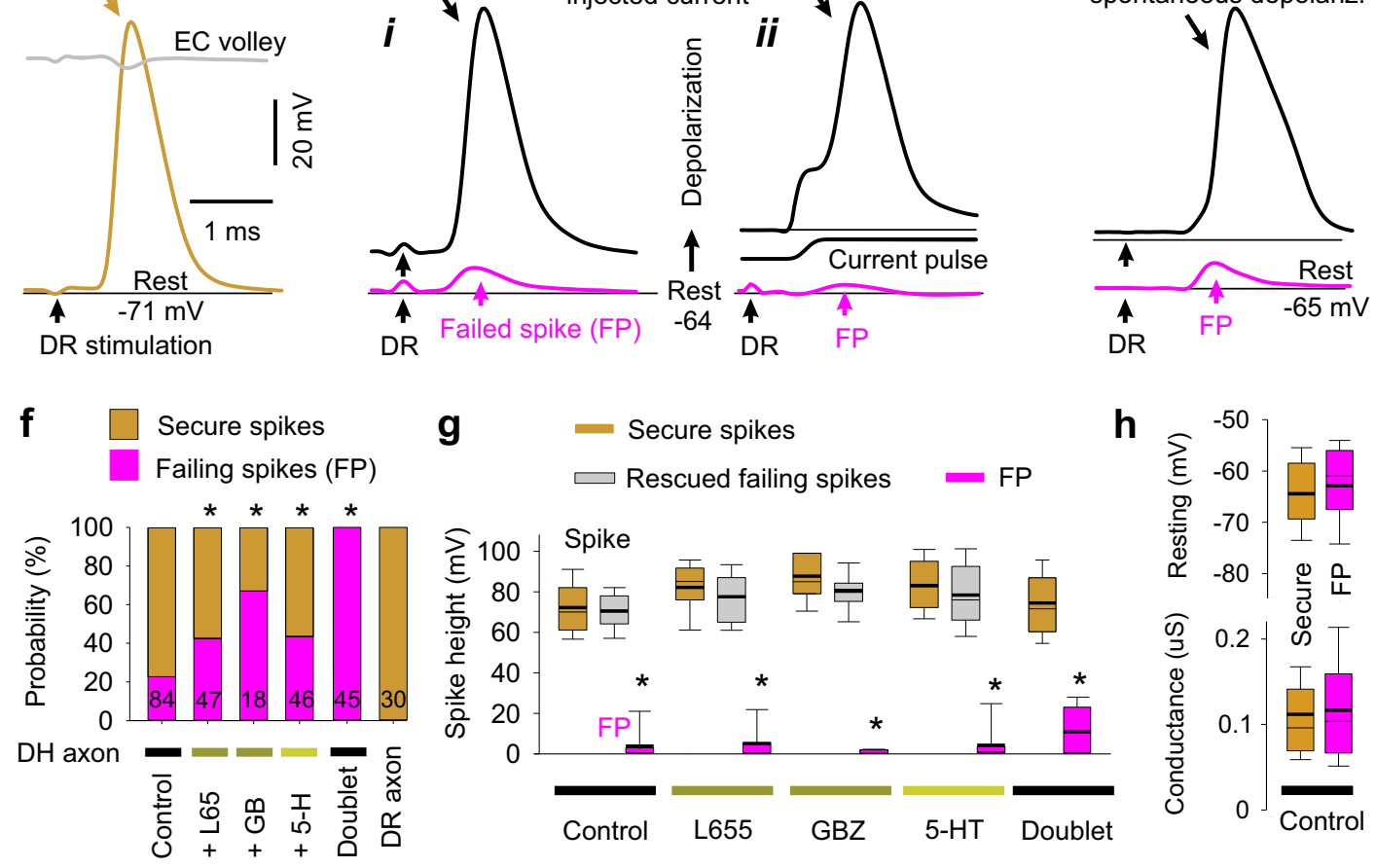

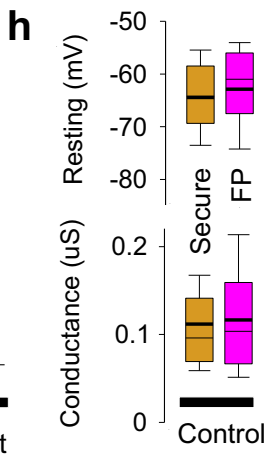


Fig. 2 | Spike failure. a, Recording from ex vivo whole adult rat spinal cord. b-d, Intracellular recordings from proprioceptive axon branches in the dorsal horn (DH), with dorsal root (DR) stimulation (1.1x threshold T, $0.1 \mathrm{~ms}$ ) evoking a spike in some branches (secure, b) and only a failed spike in others (failure potential, FPs; c, d), but depolarization restoring full spikes (black, c and d). Averages of 10 trials at $3 \mathrm{~s}$ intervals. Resting potential: thin line. EC: extracellular afferent volley. Axons from S4 sacral DR. e, Fast repeated DR stimulation induced failure in secure spikes (not failing on first stimulation, 1.1xT). Threshold interval (longest) for failure (FP, pink), and just prior to threshold (gold). f, Proportions of DH axon branches (or DR axons) failing to spike with DR stimulation under control resting conditions and with L655708 $(0.3 \mu \mathrm{M})$, gabazine (50 $\mu \mathrm{M}$; GBZ), 5HT $(10 \mu \mathrm{M})$ or fast repetition (doublet, e). *significantly more than control, $\chi$-squared test, $P<0.05, n$ indicated in bars. $\mathbf{g}$, Summary of spike and FP heights in secure and failing branches. Box plots: median, thin line; mean, thick line; interquartile range IQR, box; extreme data points within $1.5 \times \mathrm{IQR}$ of box, error bars. * significantly smaller than secure spike, $P<0.05$, for spikes in $(\mathbf{f})$. $\mathbf{h}$, Resting membrane potential and conductance for secure and failing branches, not significantly different, $P>$ 0.05 , for control axons in (f). 
bioRxiv preprint doi: https://doi.org/10.1101/2021.01.20.427494; this version posted March 28, 2021. The copyright holder for this preprint (which was not certified by peer review) is the author/funder. All rights reserved. No reuse allowed without permission.

a

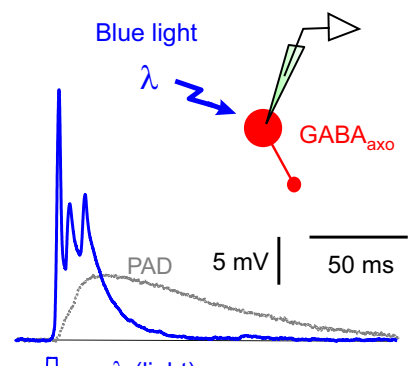

b Sensory axon depolarized by light

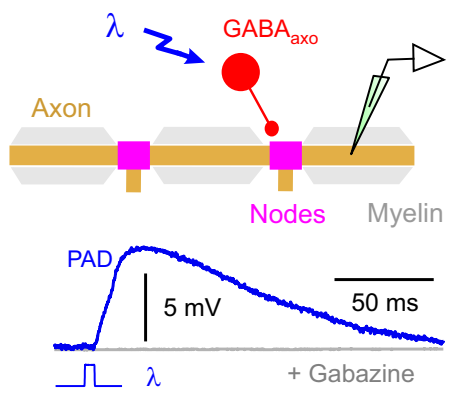

$\mathrm{GABA}_{\mathrm{A}}$ receptor block

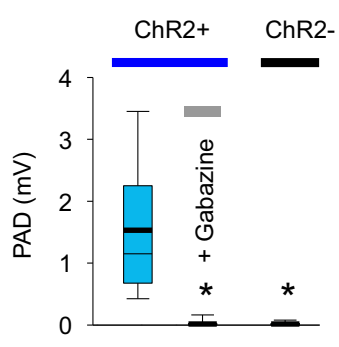

d

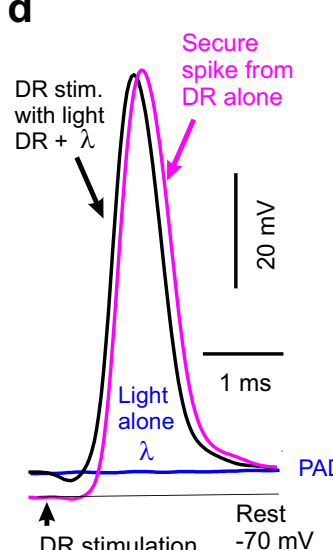

DR stimulation $\quad-70 \mathrm{mV}$

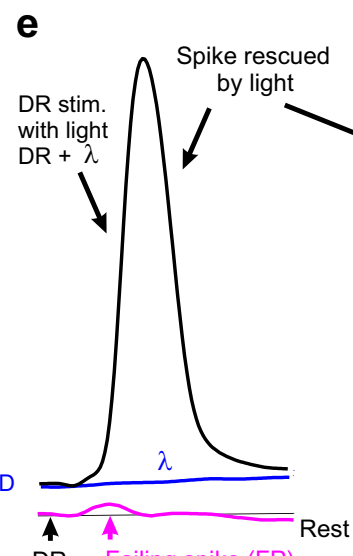

DR Failing spike (FP)

\section{f}

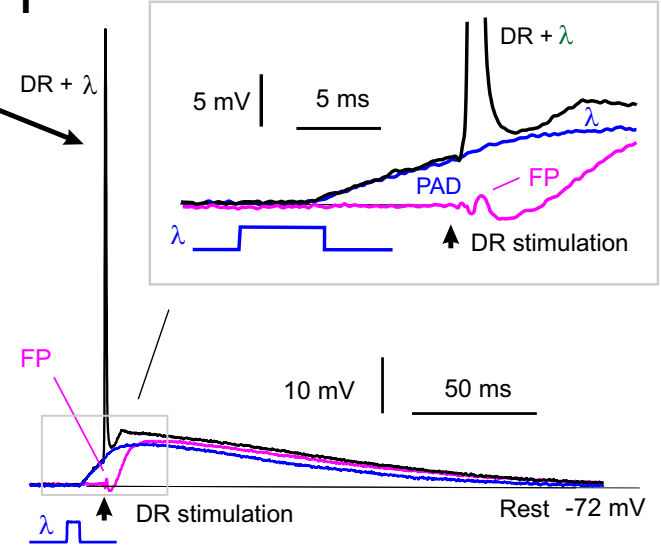

C

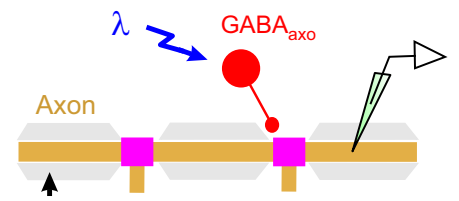

DR stim.

Spike $\bigwedge \longrightarrow \underbrace{\begin{array}{c}\text { Failing } \\ \text { node }\end{array}}$ g Spike rescue by GABA

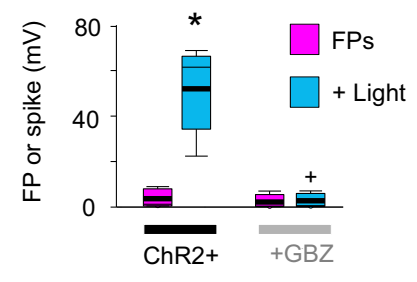

h Incidence of spike failure

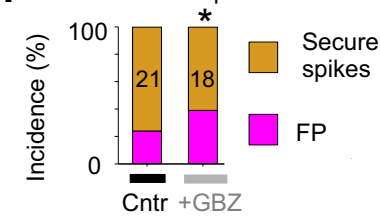

i Nodal GABA axo

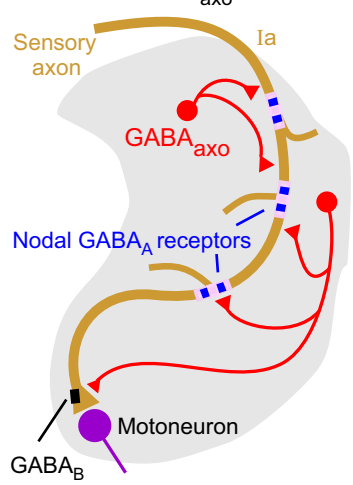

j
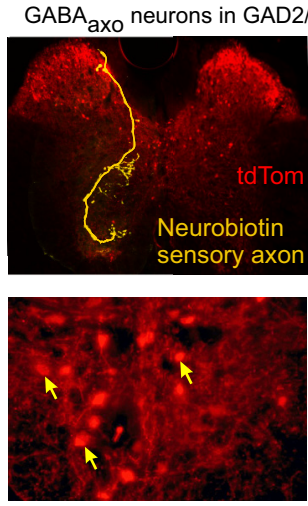
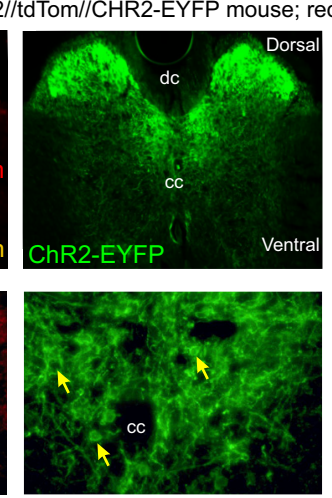
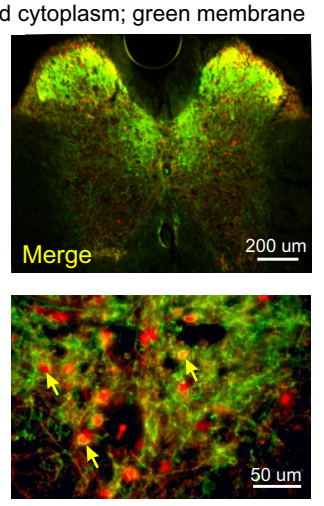

$\mathbf{k}$

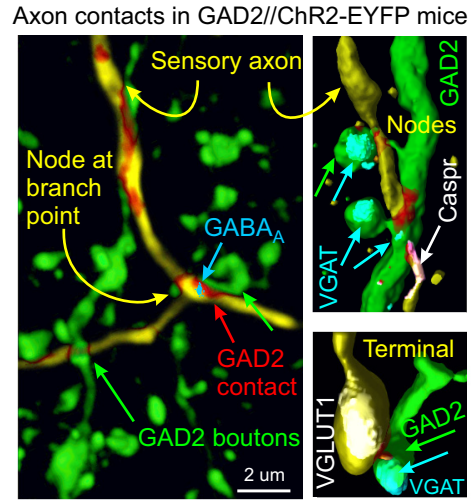

Raw image stack
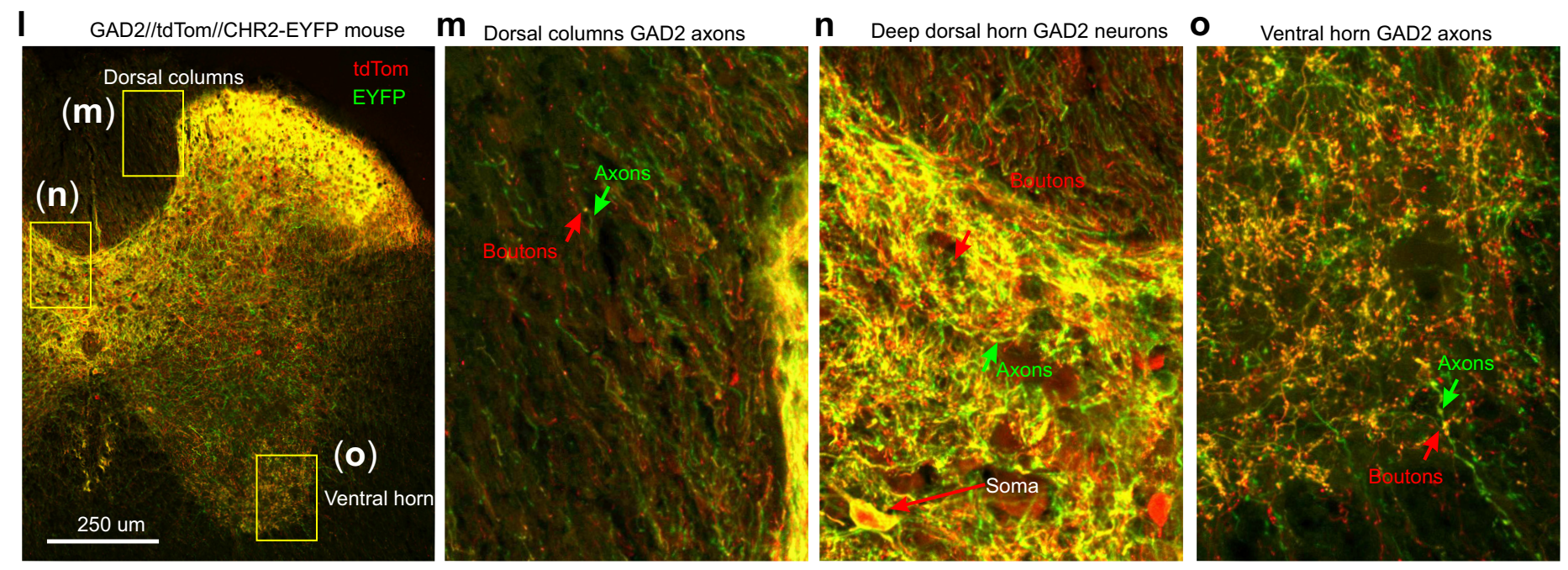
Fig. 3 | Nodal facilitation by GABA axo neurons. a, Intracellular recording from GABAaxo neuron in ex vivo spinal cord of GAD2//ChR2-EYFP mouse, with ChR2 activated with a light pulse (5 ms, $\lambda=$ $447 \mathrm{~nm}$ laser, $0.7 \mathrm{~mW} / \mathrm{mm}^{2}, 1.5 \mathrm{x}$ light threshold, T) causing a long depolarization and asynchronous spiking; isolated in $50 \mu \mathrm{M}$ gabazine ( $n=5 / 5$ similar). Average of 10 trials at $0.3 \mathrm{~Hz}$, blue. PAD from (b), grey. Resting at $-61 \mathrm{mV}$. b, Intracellular recording from proprioceptive axon branch (in $\mathrm{DH}$, sacral S3) with light pulse $(1.5 \times \mathrm{T})$ producing a long depolarization $(\mathrm{PAD}, n=14)$. Average of 10 trials at 0.3 Hz. * significantly less with gabazine or omitting ChR2 (control mice), $n=12$ each. Resting at $-71 \mathrm{mV}$. c-g, DR stimulation at rest (1.1xT) evoked a secure spike in some axon branches (d) and not others (e, f, FPs; DH S3 axons). Light evoked PAD ( $\lambda, 1.5 \times \mathrm{x}, 10 \mathrm{~ms}$ prior) rescued failed spikes (e, f) and sped up conduction in secure spikes (d). Box plots of FPs and spikes (g); *significant increase with light, $n=$ $10 ;+$ significant reduction in light effect with $50 \mu \mathrm{M}$ gabazine, $n=7 . \mathbf{h}$, Incidence of branches with failed DR-evoked spikes. *significant change with gabazine, $\chi$-squared test, $P<0.05, n$ indicated.

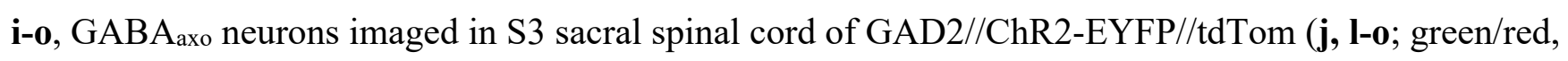
merge yellow) or GAD2//ChR2-EYFP (k, green, dorsal horn) mice. Innervation of 3D reconstructed neurobiotin filled sensory axons (gold in $\mathbf{j}$ and $\mathbf{k}$, as in Fig. 1) by GABAaxo neurons (green; axon contacts labelled red in $\mathbf{k}$ ) in dorsal horn. Nodes identified by Caspr and paranodal taper, sensory terminals by VGLUT1, GABA axo terminals by VGAT, and axonal GABAA receptors by the $\alpha 5 \mathrm{GABA}_{\mathrm{A}}$ subunit. ChR2-EYFP is mainly expressed on plasma membranes ${ }^{107}$, whereas tdTom is cytoplasmic, and so tdTom rings EYFP labelling, especially evident on the soma and boutons (j, l-o). Regions in (l) expanded in (m-o). 
a Intracellular axon recording
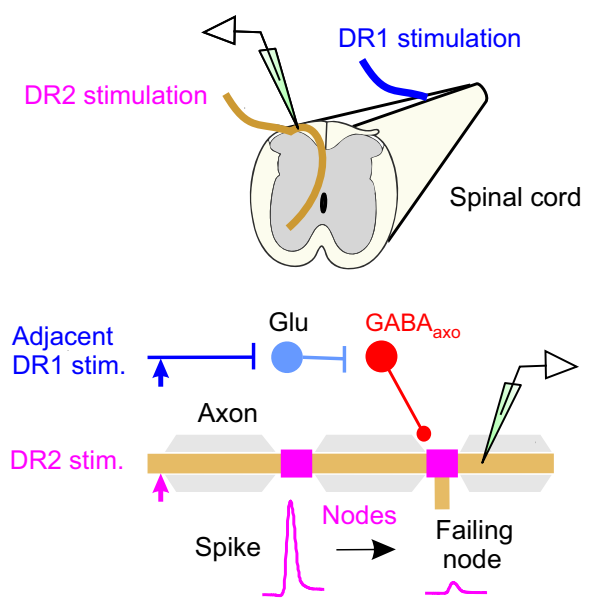

b

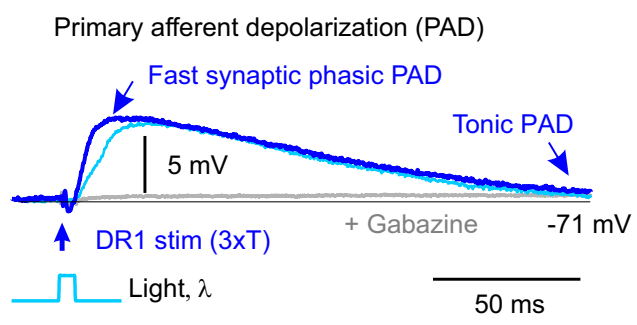

C Composite PAD recorded from dorsal root (DR2)

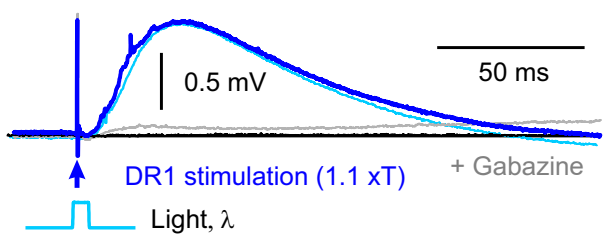

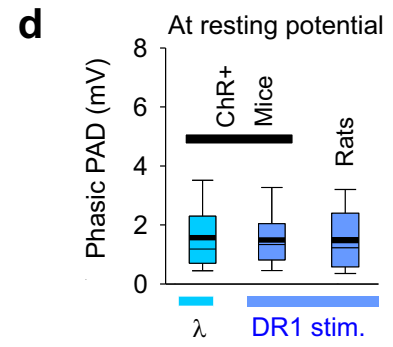

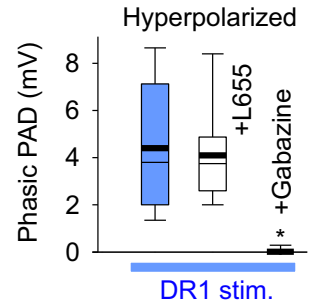

e
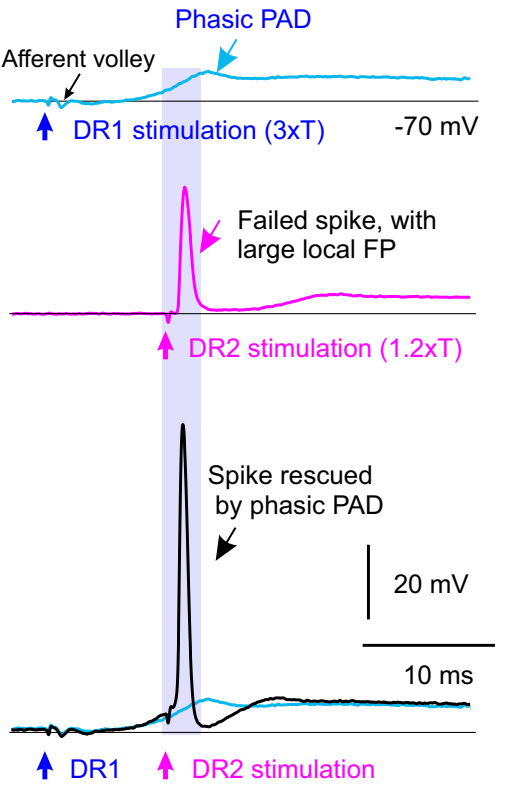

f

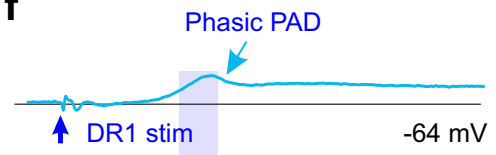

Failed spike, with very small distal FP

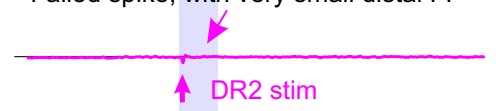

Spike rescued by phasic PAD

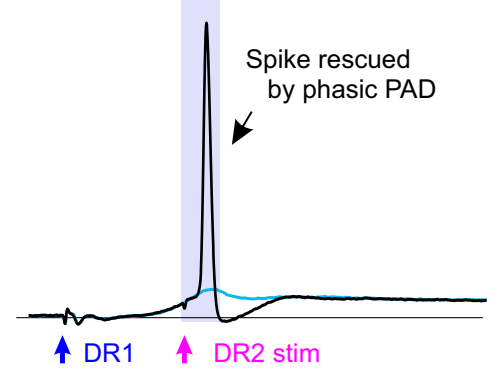

g

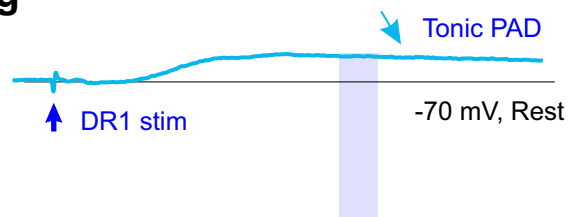

Failed spike, with small distal FP

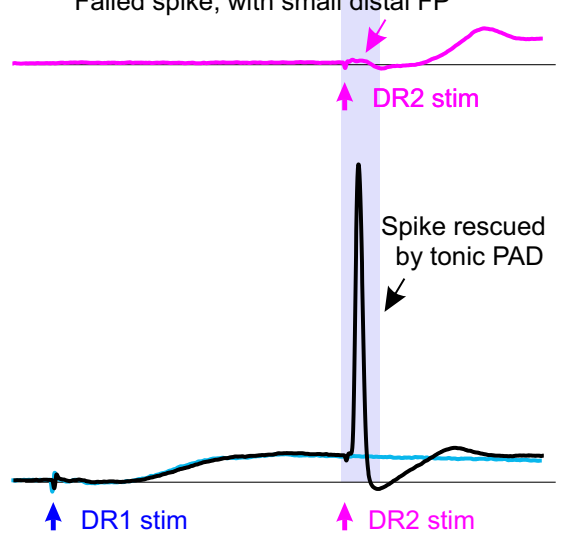

h

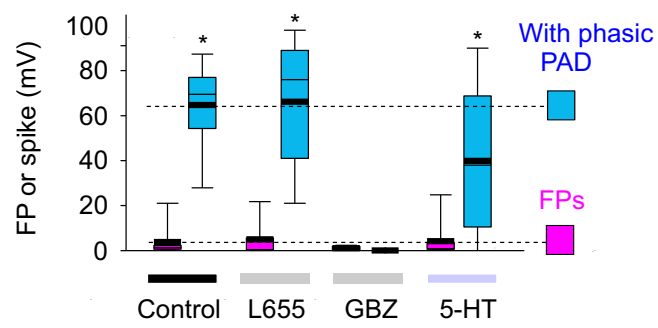


Fig. 4 | Sensory driven nodal facilitation. a, Experimental setup to indirectly activate GABAaxo neurons by DR stimulation (DR1), via a trisynaptic circuits detailed in Extended Data Figs. 5-6, in ex vivo spinal cords of rats or GAD2//ChR2-EYFP mice. b-c, Depolarization of a proprioceptive axon branch (b, intracellular in sacral S3 DH) or multiple axons in a DR (c, grease gap recording; sacral S3 DR; DR2) from stimulating the adjacent S4 DR (1.1xT, $0.1 \mathrm{~ms}$ pulse; DR1) or applying a light pulse to activate GABAaxo neurons $\left(5 \mathrm{~ms}, 447 \mathrm{~nm}, 0.7 \mathrm{~mW} / \mathrm{mm}^{2}\right.$, as in Fig. $\left.3 b\right)$, both blocked by gabazine (50 $\mu \mathrm{M}$; PAD), in GAD2//ChR2 mouse. Thin line resting potential. d, Summary box plots of peak phasic PAD evoked in axons by adjacent DR stimulation (DR1) or light, at rest (top, rats and mice, $n=14$ each) and with hyperpolarization $(-10 \mathrm{mV}$, bottom, same rats), and effects of applied gabazine (50 $\mu \mathrm{M}$; $n=14$ rats) or L655708 (0.3 $\mu \mathrm{M} ; n=8$ rats). *significant difference from pre-drug (blue, lower plot), $P$ $<0.05$. e-g, DR axon branches (sacral S3 DH) exhibiting spike failure (FPs, magenta) following stimulating their DR (S3 DR, 1.1xT, $0.1 \mathrm{~ms}$; DR2) in rats at rest. Spikes rescued by PAD evoked by prior conditioning of adjacent DR (S4 or contralateral S3 DR, at 3xT; DR1). Rescue occurred with fast synaptic depolarizations (phasic PAD; e-f) and tonic depolarizations (tonic PAD, g), both for local FPs (large, e) or distal FPs (small, f-g). h, FP or spike heights before and during DR evoked phasic PAD ( $n$ $=10)$ as in e-f, and actions of L655708 $(n=9,0.3 \mu \mathrm{M})$, gabazine $(n=14,50 \mu \mathrm{M})$ and 5-HT $(n=8,10$ $\mu \mathrm{M})$ in rats. *significant increase in spike with $\mathrm{PAD}, P<0.05$. 
a

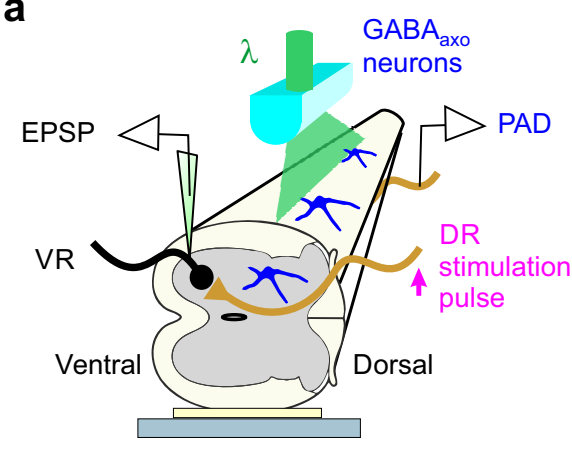

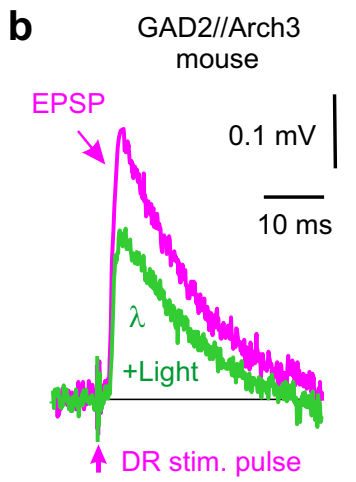

e GAD2//ChR2 mouse
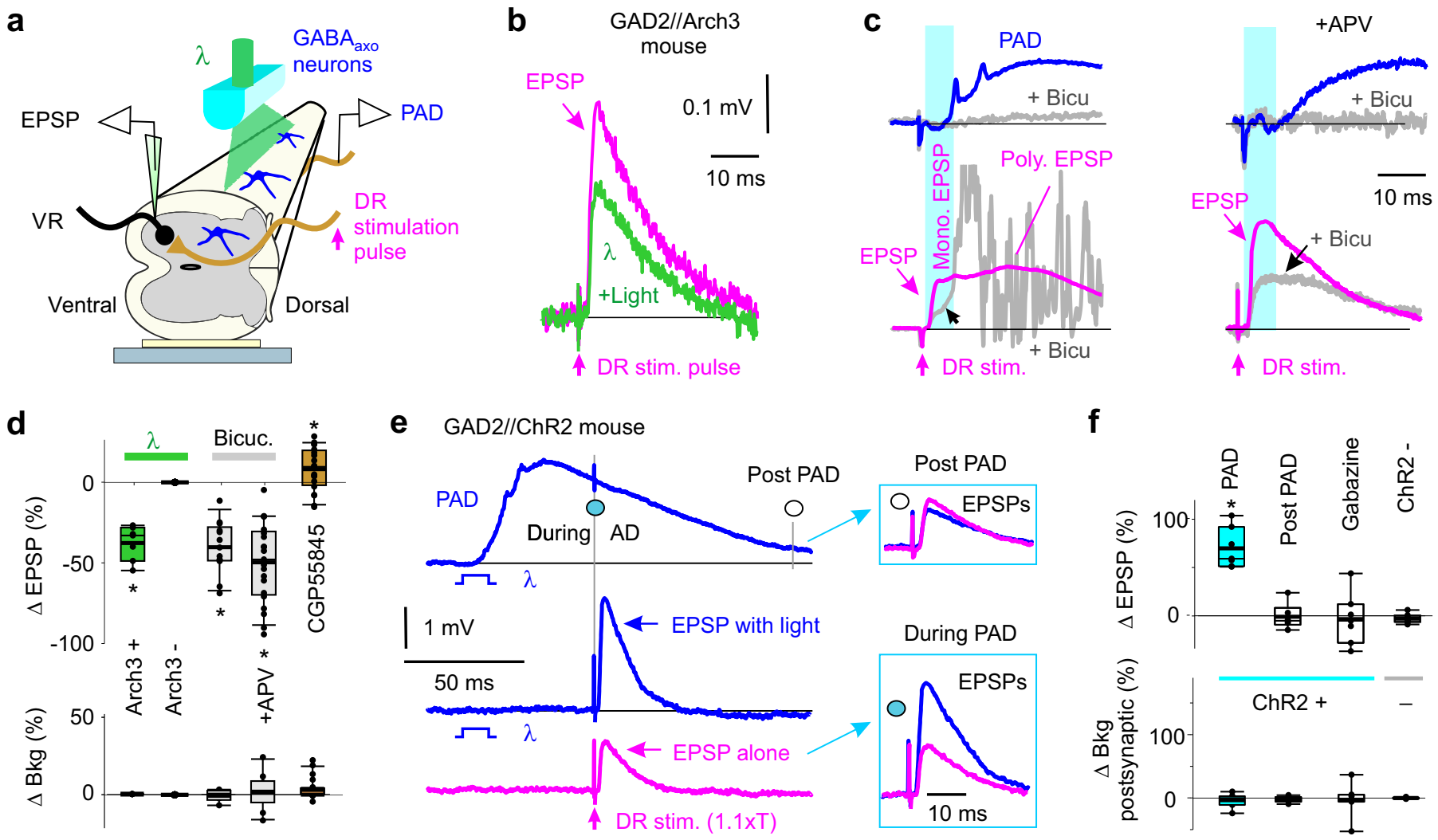

f

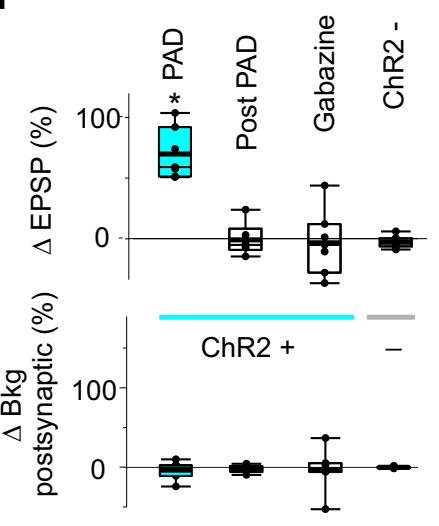

g

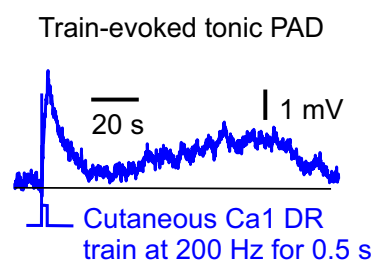

h

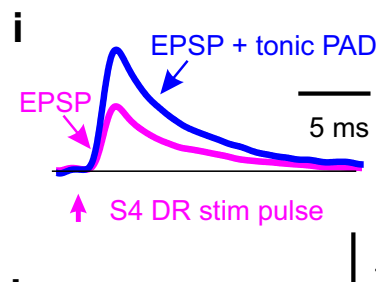

$\mathbf{k}$

+ Tonic PAD

Unit $1+2+3$

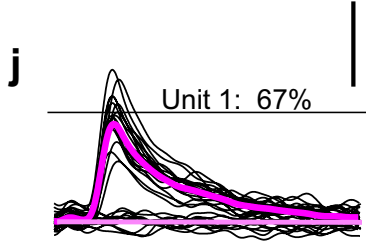

个 S4 DR stim pulse

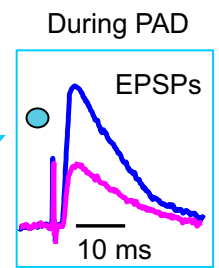

I

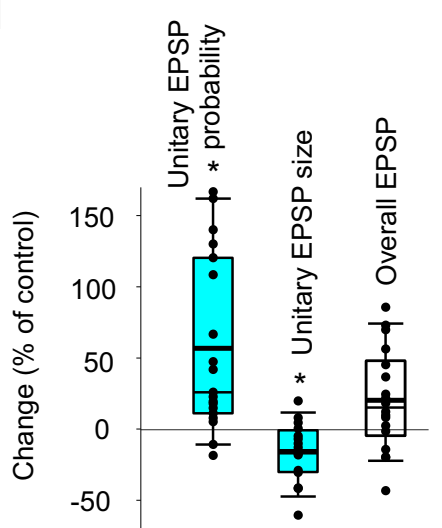


Fig. 5 | Facilitation of monosynaptic sensory transmission by GABA. a, Ex vivo recording from

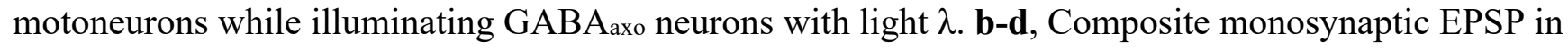
motoneurons (recorded in sacral S4 VR) evoked by a DR stimulation pulse alone (S4 DR, $0.1 \mathrm{ms,}$

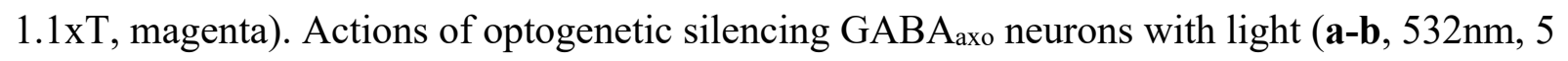
$\mathrm{mW} / \mathrm{mm}^{2}, 80 \mathrm{~ms}$, in GAD2//Arch3 mice, $\left.n=7\right)$, blocking GABAA receptors (c, with bicuculline or gabazine, $50 \mu \mathrm{M}$, with and without NMDA antagonist APV, $50 \mu \mathrm{M} ; n=23$ and 13 in rats and mice combined), or blocking GABAB receptors (d, CGP55845, $0.3 \mu \mathrm{M}, n=10$ rats and 10 mice). PAD shown for reference, recorded on S3 DR (c, top). Box plots of changes in EPSP and background postsynaptic activity (Bkg, over 10 ms prior to EPSP) with light or drugs, and with Arch3+ and Arch3$(n=5)$ mice $(\mathbf{d}) .{ }^{*}$ significant change $\Delta, P<0.05$. GAD2//Arch3 mice are VGAT + , not shown. e-f, Composite EPSP (evoked in S4 motoneurons, as in a-b) before, during and post PAD (recorded simultaneously on S3 DR) evoked by light activation of GABA axo neurons (10 ms, 1.1xT, 447nm, 0.5 $\mathrm{mW} / \mathrm{mm}^{2}, 60 \mathrm{~ms}$ and $140 \mathrm{~ms}$ pre EPSP, ISI) in GAD2//ChR2 mice. Box plots of changes in EPSP and Bkg (10 ms prior) with light in $\mathrm{ChR}^{+}$mice without and with gabazine (50 $\mu \mathrm{M}$, during PAD), and in ChR2 ${ }^{-}$mice (60 ms ISI). * significant change, $P<0.05, n=7$ each. g, Tonic PAD (L655905 sensitive) recorded in sacral S4 proprioceptive axon in response to $0.5 \mathrm{~s}, 200 \mathrm{~Hz}$ DR stimulation train applied to the largely cutaneous Ca1 DR of caudal cord (3xT, DR2) in rat. h-i, Average EPSP in S4 motoneuron (intracellular recording, EPSP evoked by S4 DR stimulation at $3 \mathrm{~s}$ intervals used for average; DR1) before and during tonic PAD evoked by the brief DR train of $\mathbf{g}$, at matched postsynaptic potentials (Bkg). $\mathbf{j}-\mathbf{k}$, Individual trials used to make EPSP averages in (i) (at $1 \mathrm{~s}$ intervals, $\mathbf{h}$ ), with large all or nothing unitary EPSPs (thick lines unitary averages; dotted single occurrence of Unit 3). Lowpass filtered at $3 \mathrm{kHz}$. l, Changes in unitary EPSP probability and size, and overall EPSP with tonic PAD. Box plots. * significant change, $P<0.05, n=18$. 


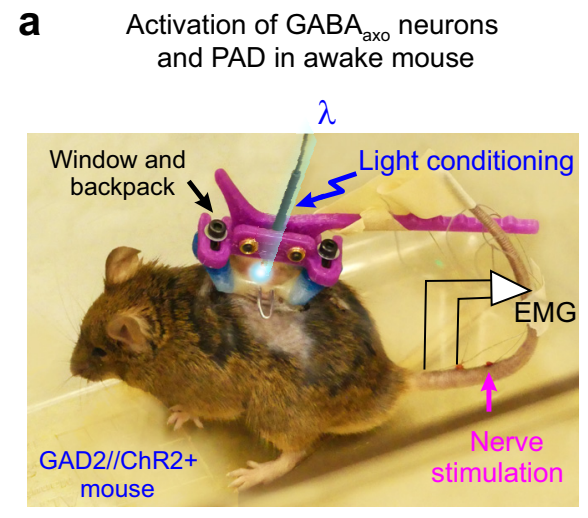

e Cutaneous conditioning to activate PAD and facilitate MSR in awake rat

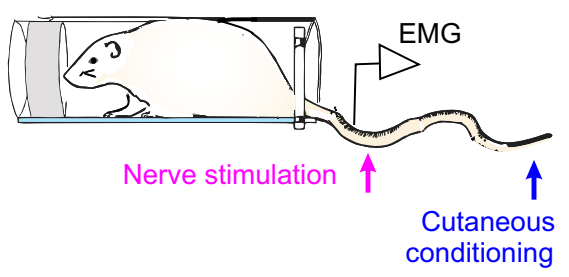

h $\quad$ 665 inhibition of MSR

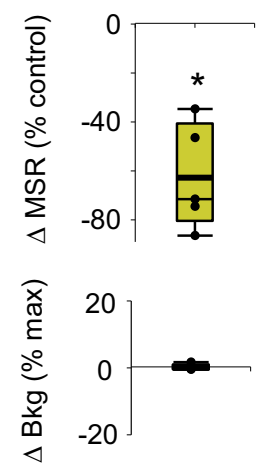

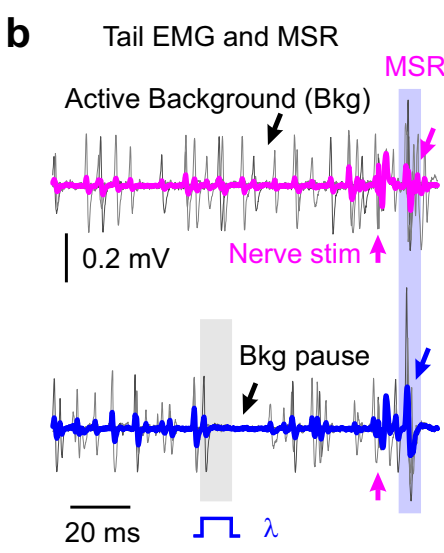

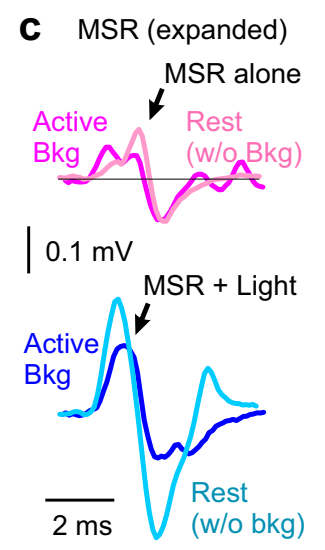

d Optogenetic facilitation of MSR

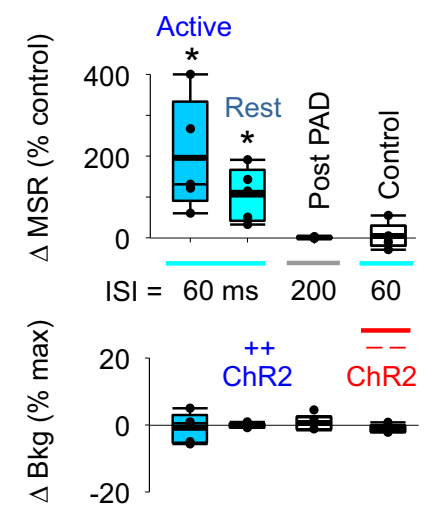

f Tail MSR with matched background EMG
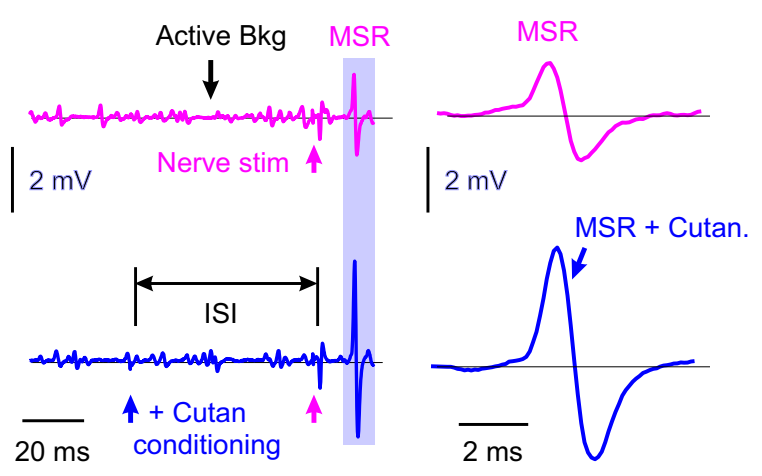

g Cutaneous facilitation of MSR

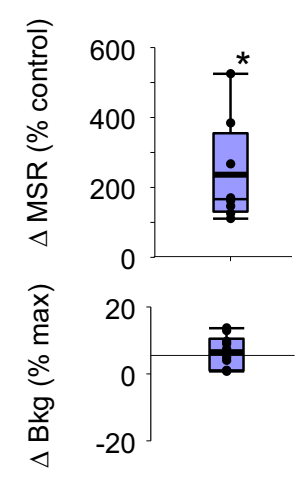

Phasic and tonic facilitation of MSR

$$
\text { Cutaneous conditioning }
$$
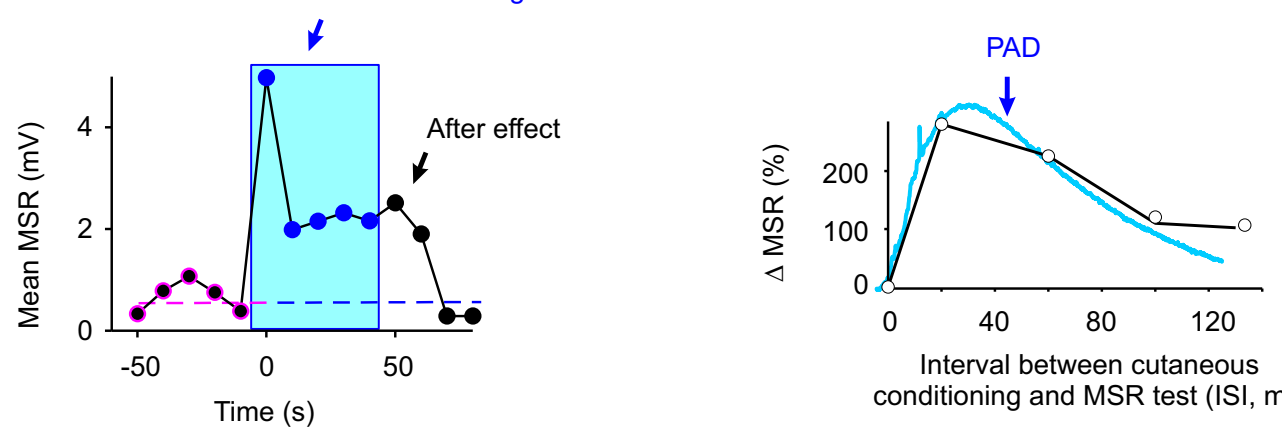

Interval between cutaneous conditioning and MSR test (ISI, ms) 
Fig. 6 | Facilitation of reflexes in awake animals. a, Recording tail muscle EMG and evoking monosynaptic reflexes (MSR) with tail nerve stimulation (1.1xT, $0.2 \mathrm{~Hz}$ ), while activating GABAaxo neurons (PAD) with light $\left(\lambda=447 \mathrm{~nm}, 10 \mathrm{~ms}\right.$ pulse, $\left.1.5 \times \mathrm{xT}, 5 \mathrm{~mW} / \mathrm{mm}^{2}\right)$ in GAD2//ChR2 mice. b-c, Effect of light pulse $\lambda$ on active background EMG (Active Bkg condition in b) and the MSR evoked 60 ms later, the latter expanded in (c). MSR tested with (b,c, Active Bkg, 30\% max) and without (c, Rest) background EMG. Thin lines in (b) are individual trial examples at $10 \mathrm{~s}$ intervals $(0.1 \mathrm{~Hz})$; thick lines: averages. d, Changes in MSR with light activation of GABAaxo neurons at matched postsynaptic background (Bkg) (over 20 ms prior to MSR; lack of change in Bkg). Measured in active and resting (no Bkg) states, in ChR2+ and ChR2- mice (rest only), and during (60 ms ISI) and post PAD (200ms ISI at rest only). ISI: interstimulus interval. Box plots. ${ }^{*}$ significant change, $P<0.05, n=5$ mice each. e-g, MSR recorded as in (b-d), but in rat and with PAD instead activated with cutaneous conditioning (tip of tail, $0.2 \mathrm{~ms}, 2 \times \mathrm{T}, 60 \mathrm{~ms}$ prior, $0.1 \mathrm{~Hz}$ repetition), at matched active Bkg EMG. * significant change, $P<0.05, n=8$ rats. $\mathbf{h}$, Decrease in MSR with L655935 (1 mg/kg i.p.) at matched Bkg EMG. Box plot. * significant change, $P<0.05, n=5$ rats. $\mathbf{i}$, Typical MSR amplitude before, during and after conditioning as in (e) with after effect. $\mathbf{j}$, Typical change in MSR with cutaneous conditioning as in (e) when the ISI is increased, compared to PAD (from Fig. 4). (i,j) similar results in $n=5 / 5$ rats. 
a

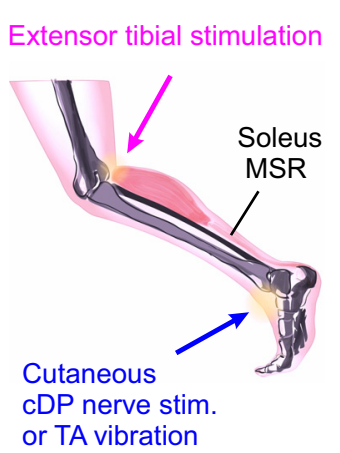

d Conditioning summary:

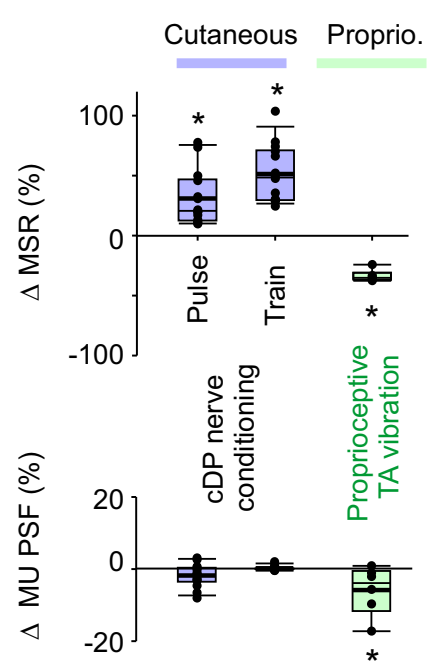

e $\quad$ i Tonic facilitation with $\mathrm{CDP}$ train
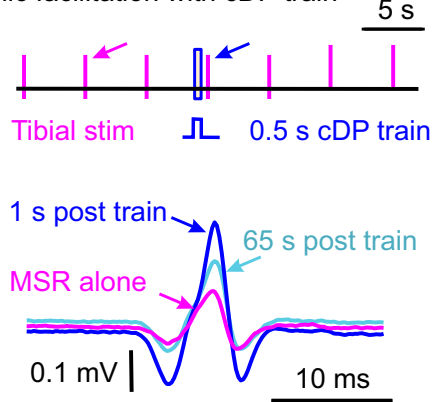

ii Tonic facilitation averages *

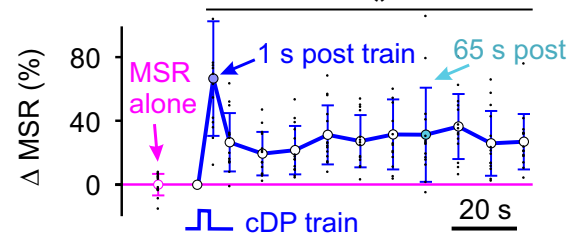

iii

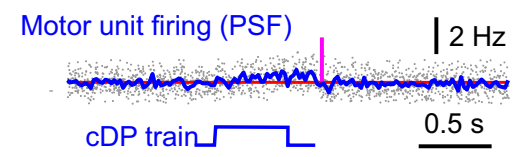

b $\boldsymbol{i}$
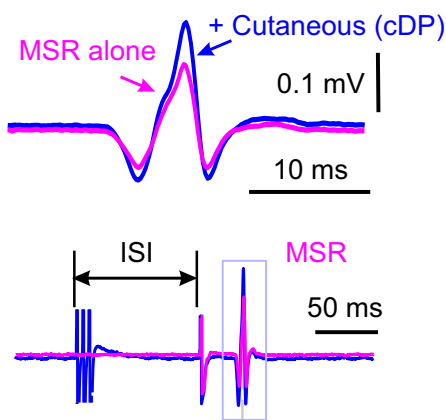

cDP stim $\uparrow \quad \uparrow$ Tibial stim

ii Phasic MSR facilitation

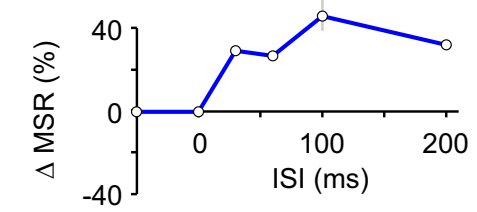

iii

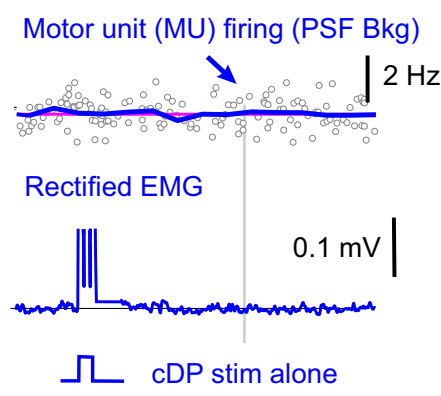

C $i$
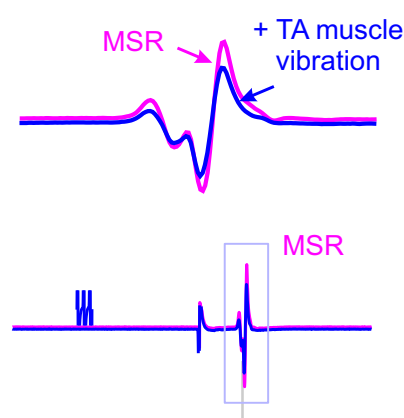

Vibration $\uparrow \quad \uparrow$ Tibial stim

ii Phasic inhibition

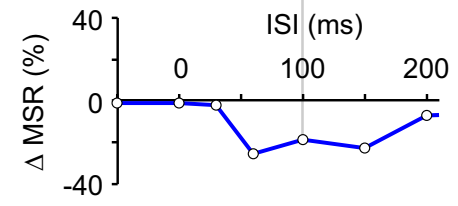

iii $\quad$ MU firing (PSF Bkg)

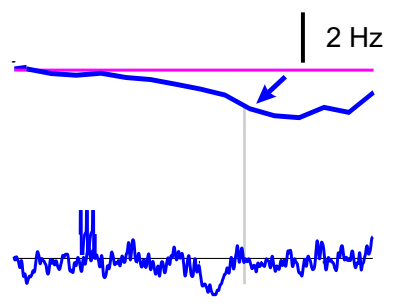

几 Vibration alone

f $i$ Motor unit firing (PSF)

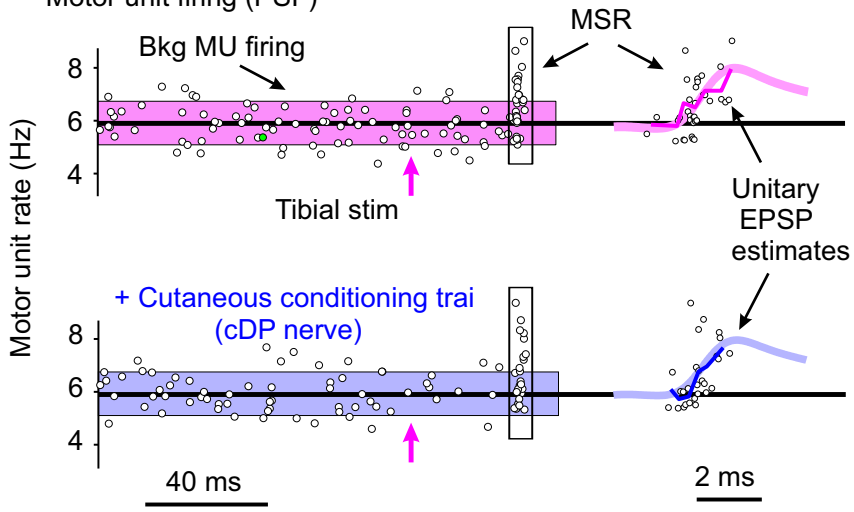

Changes in PSF estimates of unitary EPSPs and MU firing.

ii

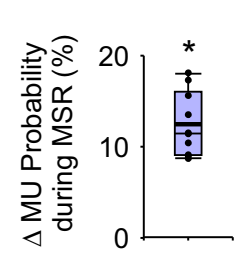

ii

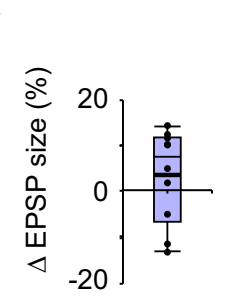

iv

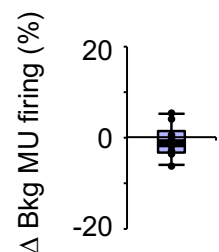


Fig. 7 | Facilitation of reflexes in humans. a-b, MSR in soleus EMG evoked by a tibial nerve pulse $(1.1 \times T, 0.2 \mathrm{~Hz}, \mathbf{b i})$, and phasic facilitation of the MSR following a brief conditioning of the cutaneous branch of the deep peroneal nerve (cDP nerve) at varying intervals (ISIs, bii, 1.0xT, perception threshold T, at rest), and lack of changes in background (Bkg) motor unit (MU) activity or EMG evoked by conditioning alone (biii, peri-stimulus frequencygram, PSF; with weak contraction). c, Same as (b), but with proprioceptive conditioning evoked by a brief tibial anterior (TA) muscle tendon vibration, which alone inhibited MU activity (postsynaptic inhibition, PSF Bkg, ciii). d, Summary box plots of changes in MSR and postsynaptic (MU) activity with brief conditioning (cDP, $n=14$ subjects; or TA vibration, $\mathrm{n}=6$; as in $\mathbf{b}-\mathbf{c})$ and long cutaneous conditioning trains $(\mathbf{e}, n=14) . *$ significant change with conditioning, $P<0.05$. e, Tonic increase in MSR (tonic facilitation) after $0.5 \mathrm{~s}$ cutaneous conditioning train (cDP, 1.1xT, $200 \mathrm{~Hz}$ ) at rest (ei-ii), without prolonged changes in MU activity induced by conditioning alone (eiii, PSF in weak contraction). MSR evoked by tibial stimulation every $5 \mathrm{~s}$, with averages from repeated conditioning shown in (eii). * significant change in MSR, $\mathrm{P}<0.05, \mathrm{n}=5$ subjects. f, Overlay of all MU firing rates (PSF) with repeated MSR testing (at $5 \mathrm{~s}$ intervals) during ongoing weak contraction, and effect of the $0.5 \mathrm{~s}$ cutaneous conditioning train (fi). Summary box plots of increased probability of MU firing during MSR (fii), without changing estimated EPSP size (fiii, PSF thin line; thick line unitary EPSP shape from Fig. 5j) or background MU firing (Bkg, fiv). * significant change with conditioning, $P<0.05, n=10$ subjects. 


\title{
Supplementary information
}

\section{GABA facilitates spike propagation at branch points of sensory axons in the spinal cord}

\author{
Krishnapriya Hari ${ }^{1,6}$, Ana M. Lucas-Osma ${ }^{1,2,6}$, Krista Metz ${ }^{1}$, Shihao Lin ${ }^{1}$, Noah Pardell, \\ David A. Roszko ${ }^{1}$, Sophie Black ${ }^{1}$, Anna Minarik ${ }^{1}$, Rahul Singla ${ }^{1}$, Marilee J. Stephens ${ }^{1,3}$, \\ Karim Fouad ${ }^{1,2}$, Kelvin E. Jones ${ }^{1,4}$, Monica A. Gorassini ${ }^{1,3}$, Keith K. Fenrich ${ }^{1,2,7}$, Yaqing \\ $\mathrm{Li}^{1,5,7}$ and David J. Bennett ${ }^{1,2,7,8^{*}}$
}

\footnotetext{
${ }^{1}$ Neuroscience and Mental Health Institute, University of Alberta, Edmonton, AB, T6G 2R3, Canada. ${ }^{2}$ Faculty of Rehabilitation Medicine, University of Alberta, Edmonton, AB, T6G 2G4, Canada. ${ }^{3}$ Department of Biomedical Engineering, Faculty of Medicine and Dentistry, T6G 2V2, University of Alberta, Edmonton, AB, Canada. ${ }^{4}$ Faculty of Kinesiology, Sport and Recreation, University of Alberta, Edmonton, AB, T6G 2H9, Canada. ${ }^{5}$ Present address: Department of Physiology, Emory University, Atlanta, GA, 30322, USA. ${ }^{6}$ These authors contributed equally. ${ }^{7}$ Senior author. ${ }^{8}$ Lead Contact. ${ }^{*}$ Corresponding author:

bennettd@ualberta.ca.
} 

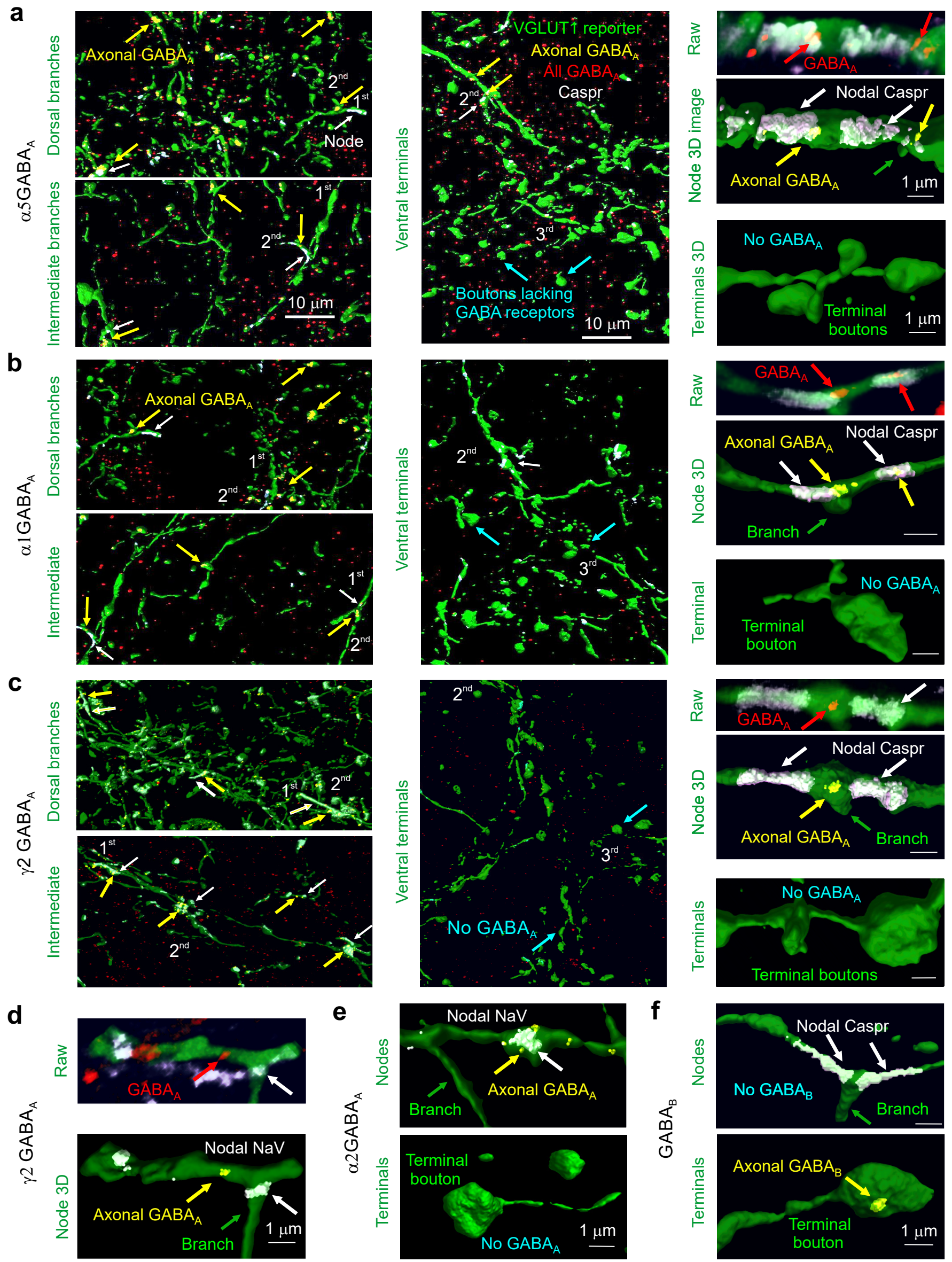


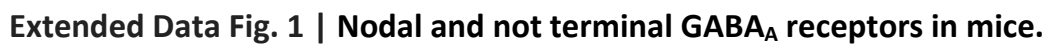

a-c, In the sacrocaudal spinal cord of mice we examined the distribution of $\mathrm{GABA}_{A}$ receptor subunits on nodes and terminals of sensory axons, including extrasynaptic $\alpha 5$ subunits, synaptic $\alpha 1$ and $\alpha 2$ subunits, and ubiquitous $\gamma 2$ subunits (e.g. forming the common $\alpha 1 \beta \gamma 2$ or $\alpha 5 \beta \gamma 2$ receptors, though less common extrasynaptic $\alpha 1 \beta \delta$ have been reported) ${ }^{32,108-}$

${ }^{110}$. We genetically labelled primary sensory axons by their expression of the vesicular glutamate transporter VGLUT1 with a

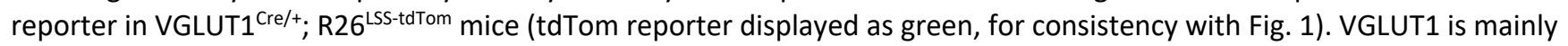
only expressed in sensory axons ${ }^{59}$, especially ventral proprioceptive afferents, as other afferents do not reach the ventral horn ${ }^{16}$. Axons were reconstructed in 3D and branching order is indicated $\left(1^{\text {st }}, 2^{\text {nd }}\right.$ and $\left.3^{\text {rd }}\right)$, as detailed in Fig. 1 . Typical axon branching patterns are shown in the deep dorsal horn (left), intermedial laminae and ventral horn (middle). Expanded images of nodes of Ranvier identified by paranodal Capsr immunolabelling are shown on $1^{\text {st }}$ order branches in the intermediate laminae (on right), along with raw confocal images prior to 3D reconstruction. Expanded terminal boutons from the ventral horn are also shown on the right. As in rats, nodes are often near branch points (where $2^{\text {nd }}$ order branch arises from $1^{\text {st }}$ order branches in this case, green arrow). We identified axonal GABA receptors as those colocalized with axonal tdTom (yellow, as in Fig. 1), and the remaining GABA receptors are labelled red in the 3D reconstructions. The $\alpha 5$, $\alpha 1$, and $\gamma 2 \mathrm{GABA}_{A}$ receptors subunits were found on large axon branches ( $1^{\text {st }}$ and $2^{\text {nd }}$ order) in the dorsal, intermediate, and ventral cord, near nodes counterstained with Caspr immunolabelling (white, arrows), but not on ventral horn terminal boutons ( $3^{\text {rd }}$ order; examples at blue arrows; expanded on right). The presence of these subunits is consistent with their mRNA previously reported in the dorsal root ganglion ${ }^{111}$. Also, the finding of $\alpha 1$ subunits on these axons is consistent with the recent observation that $\alpha 1$ is only on myelinated sensory axons, rather than unmyelinated $\mathrm{C}$ fibres ${ }^{112}$. Similar results were obtained from $n=3$ mice. All terminals are VGLUT1 ${ }^{+}$(not shown). d, Immunolabelling for $\gamma_{2}$ GABA receptor subunits on $3 \mathrm{D}$ reconstructed sensory axons, with same format and mice of (a), but with nodes identified by Nav (sodium channels), rather than Caspr. Again, the node and GABA receptors are near a branch point (green arrow). e, Immunolabelling for $\alpha 2$ $\mathrm{GABA}_{\mathrm{A}}$ receptors on $3 \mathrm{D}$ reconstructed sensory axons, with same format and mice of (a), but nodes identified by Nav. Again, $\mathrm{GABA}_{A}$ receptors were near nodes and not on terminals. f, Immunolabelling for $G A B A_{B}$ receptors on 3D reconstructed sensory axons, with same format and mice of (a). GABA receptors were generally absent from nodes identified by Caspr, but present on ventral terminal boutons, as in rats (Fig. 1). 


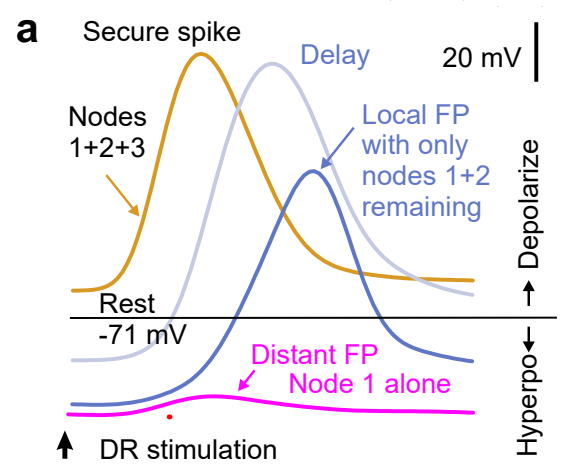

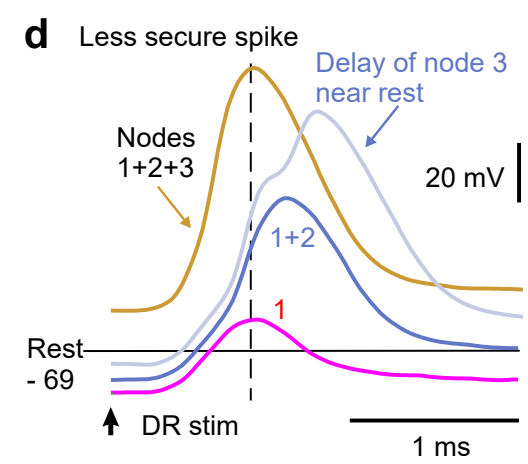

g

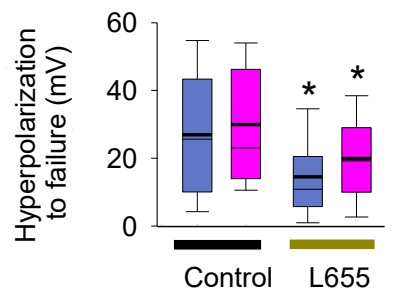

h

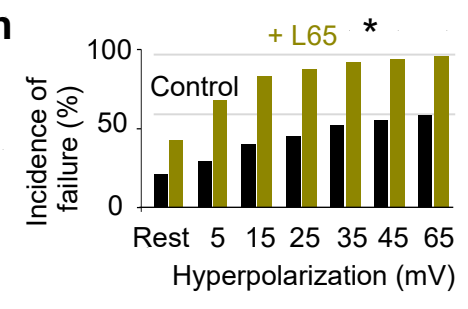

Extended Data Fig. 2 | Voltage dependence of nodal spike propagation failure following dorsal root stimulation.

a-d, Intracellular recording from proprioceptive la afferent branches in the dorsal horn with secure spikes at rest, evoked by DR stimulation (1.1xT, $0.1 \mathrm{~ms}$; sacral S4 DR; a, d). Spike failure was induced by increasing hyperpolarization (failure near rest in $\mathbf{d}$, but not $\mathbf{a}$ ), with a delay and then abrupt loss of height, reflecting failure of successively further away nodes (a, $d)$. Of the two local nodes adjacent to the electrode, one failed first with hyperpolarization, leaving the attenuated spike from the other node (local FP, about $1 \lambda_{s}$ away), which eventually failed as well with further hyperpolarization, leaving a much smaller FP from more distal nodes (distal FP). Spike failure in a node was always proceeded by a delay in the nodal spike. Estimated contributions from local nodes (b-c) were computed by subtraction from traces in (d). Note that due to attenuation of injected current with distance, larger hyperpolarizations were needed to stop spikes with more distal vulnerable nodes (secure spikes), as observed by smaller distal FPs (a verses $\mathbf{d}$ ), and some axon spikes could not be stopped (not shown; Spike only, quantified in e). Data collected in discontinuous current clamp (DCC) mode so electrode rectification during the injected hyperpolarizing current did not affect potential (DCC switching rate $7 \mathrm{kHz}$, low pass filtered at $3 \mathrm{kHz}$ to remove switching artifact, which also removed stimulus artifact). e, Distribution of spiking in branches with full spikes only, one local nodal spike (local FP), or a distal nodal spike (distant FP) remaining after maximal hyperpolarization, and * significant change when blocking $\alpha 5 \mathrm{GABA}_{\mathrm{A}}$ receptors with $\mathrm{L655708}(0.1-0.3 \mu \mathrm{M})$ using $\chi$-squared test, $P<0.05 ; n=$ 68 control and $n=47 \mathrm{~L} 655708$ treated branches. f, Box plots of spike or FP heights and delays in branches indicated in (e), measured just prior to spike failure (or at maximal hyperpolarization for secure spikes) and after failure (for local and distal FPs, as induced in a-d). Delay measured relative to peak of spike at rest. + FP significantly different than spike at rest, $P<$ 0.05. g, Box plots of the hyperpolarization needed to induce failure, in branches from (e). ${ }^{*}$ significant change with L655708, $P<0.05$. h, Incidence of failure at varying potentials (\% of total spikes from e). * significant change with L655708, $\chi$-squared test, $P<0.05$. 
a

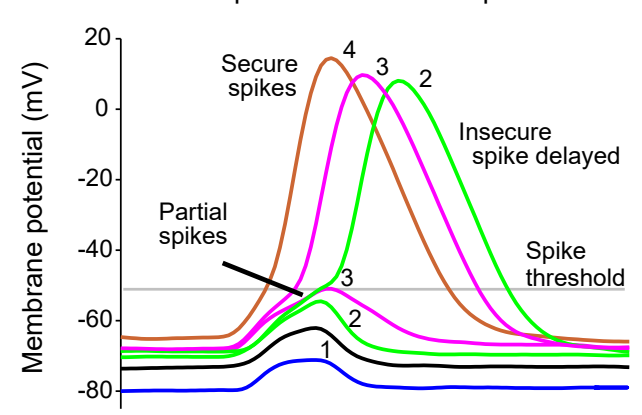

b

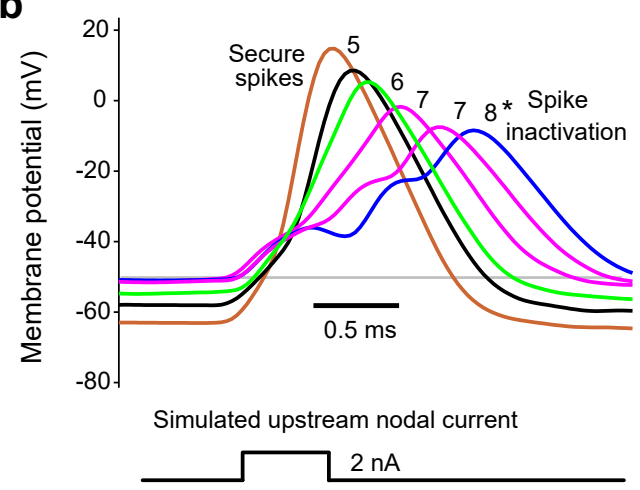

C

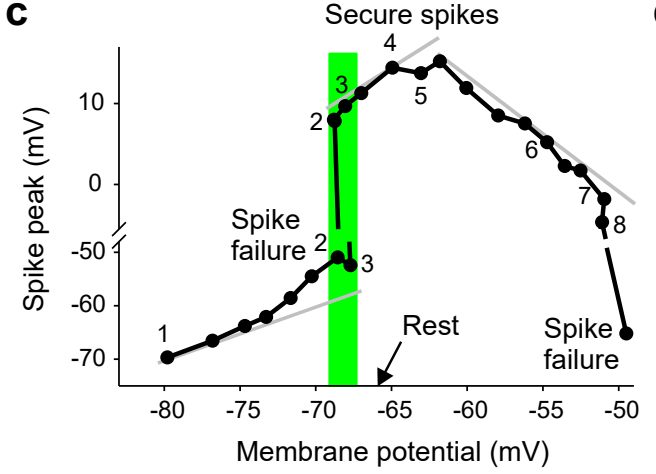

e

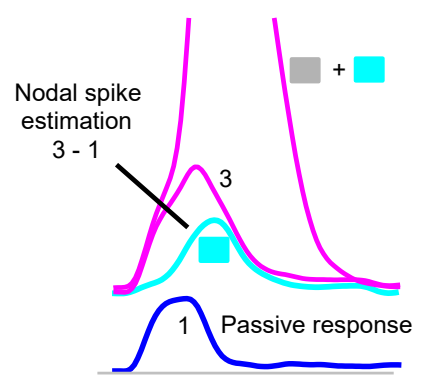

d Local control of nodal spikes

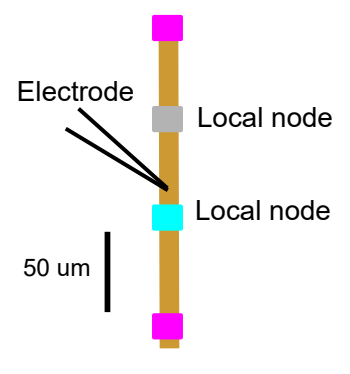

f Nodal spike separation at failure

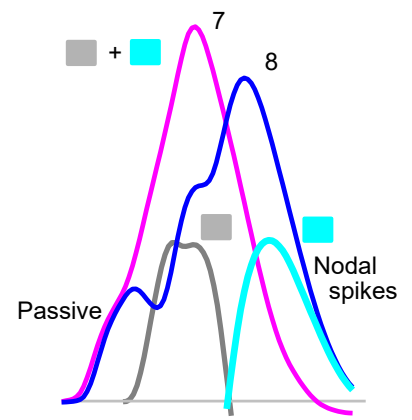

Extended Data Fig. 3 | Voltage dependence of spike failure with simulated nodal currents.

Simulating spike propagation failure in a proprioceptive axon by applying a brief intracellular current injection to mimic the current arriving from an upstream node (and FP), yielded full spikes evoked at rest, but nearby nodal spikes delayed and then failing as the membrane was held progressively more hyperpolarized with a steady bias current. Also, large steady depolarizations inactivated these spikes, though well outside of the physiological range (>-50 mV). a, Intracellular recording from proprioceptive la afferent branch in the dorsal horn (sacral S4 axon). A brief current injection pulse (0.5 ms) was applied to simulate the current arriving from distal nodes during normal axon spike conduction, and repeated at $1 \mathrm{~s}$ intervals. During these pulses the membrane was held at varying potentials for $1-2 \mathrm{~s}$ with steady current injection, with numbers and colours denoting a given holding potential (in DCC mode). At the most hyperpolarized levels spikes failed to be evoked and only the passive response is seen, like a FP (blue, 1). As the potential was depolarized to near the axon's resting potential $(-67 \mathrm{mV}$ ) partial spikes occurred (green, 2 and 3), likely from a single adjacent node activating, and then delayed broad spikes occurred, as both adjacent nodes were activated. At more depolarized levels the spikes arose more rapidly and increased in height to full secure spikes (4). $\mathbf{b}$, In the same axon as (a), at holding potentials well above those seen physiologically (near $-50 \mathrm{mV}$, lower plots) spikes started to exhibit sodium channel inactivation and failure, with a decrease in spike height and delay $(7-8)$ and eventually full failure (shown in c). Adjacent nodes started failing at slightly different times with different delays, broadening the spike and eventually separating into two distinct nodal spikes $\left(8^{*}\right)$. c, Spike heights plotted as a function of holding potential, including those spikes illustrated in ( $\mathbf{a}$ and $\mathbf{b}$ ), with spike numberlabels indicated. Left grey line indicates passive leak current response, and shows deviation from passive response near rest. Shaded green region shows all or nothing failure or spikes near the resting potential. Middle grey line shows a region of secure spikes with relatively invariant spikes. Right grey line shows spike inactivation with large depolarizations and outright failure near - $50 \mathrm{mV}$. Note the split vertical axis. Similar voltage dependence of spike failure occurred for $n=5 / 5$ axons tested. This demonstrates two modes of spike failure: 1) spikes that fail at rest or at hyperpolarized potentials and 2) spikes that fail with large depolarizations above rest. The latter is likely not physiological, since even the largest PAD that we have observed (5-10 mV; Fig. 4d) does not depolarize axons to $-50 \mathrm{mV}$, since axons rest near $-70 \mathrm{mV}$, and PAD is only large at hyperpolarized levels (Fig. 4d) and decreases steeply as the potential approaches the reversal potential for chloride $(-15 \mathrm{mV})^{16} . \mathbf{d}$, Schematic of recording arrangement and relation to adjacent nodes for data in (e) and (f). e, Expansion of responses 1 (blue) and 2 (pink) from (a), and difference (cyan) to show the first local nodal spike height at threshold, recorded at electrode. Active nodes from schematic in (d) shown with shaded boxes. f, Spikes near sodium inactivation from (b) (7 and 8), with differences indicating local nodal spikes (cyan and grey, nodes from d). 
a

Axon model

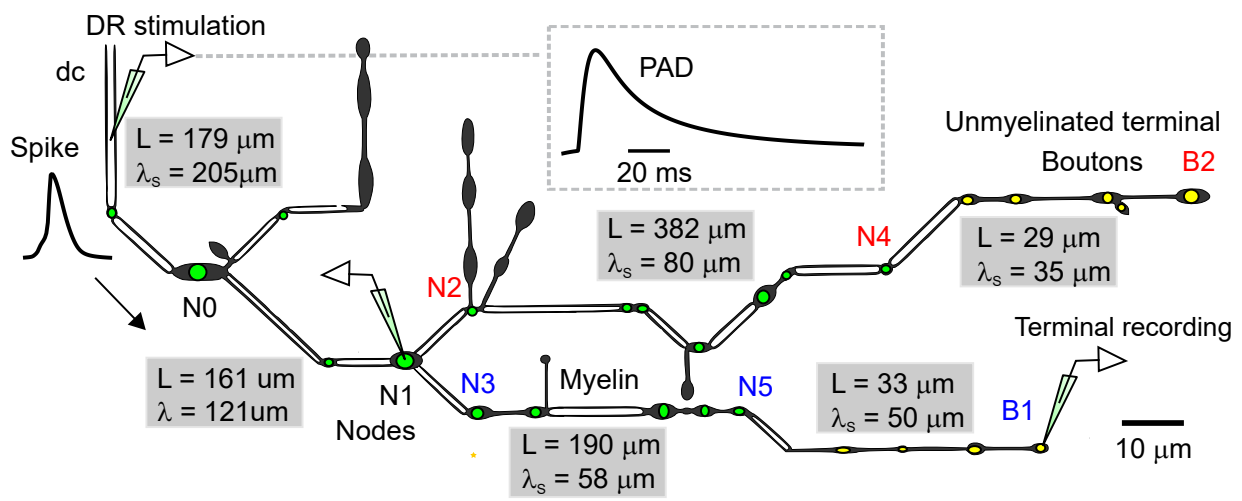

b

Spikes and FPs at proximal nodes

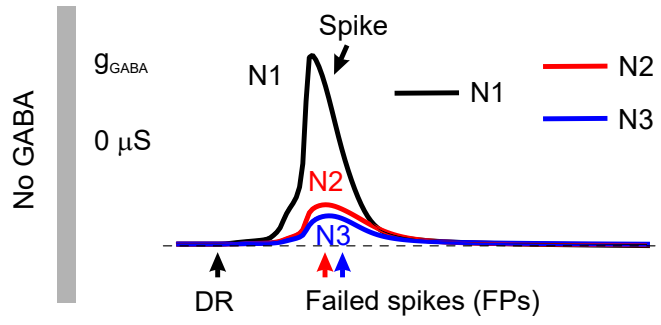

Rest

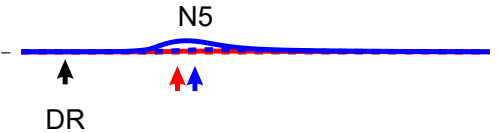

C

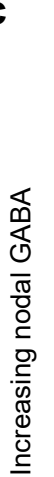
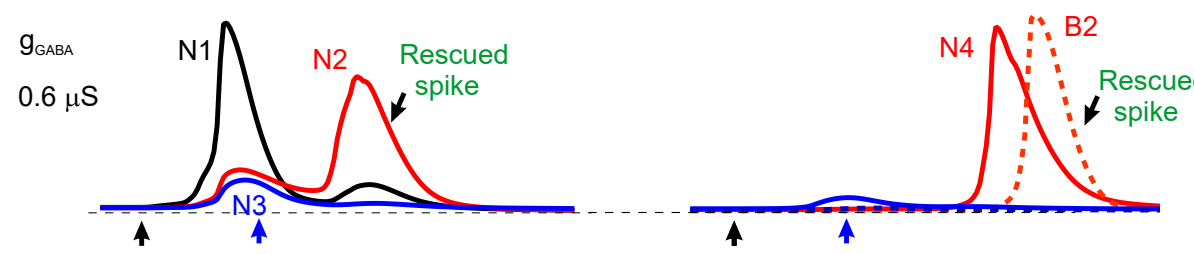

$1.5 \mu \mathrm{S}$
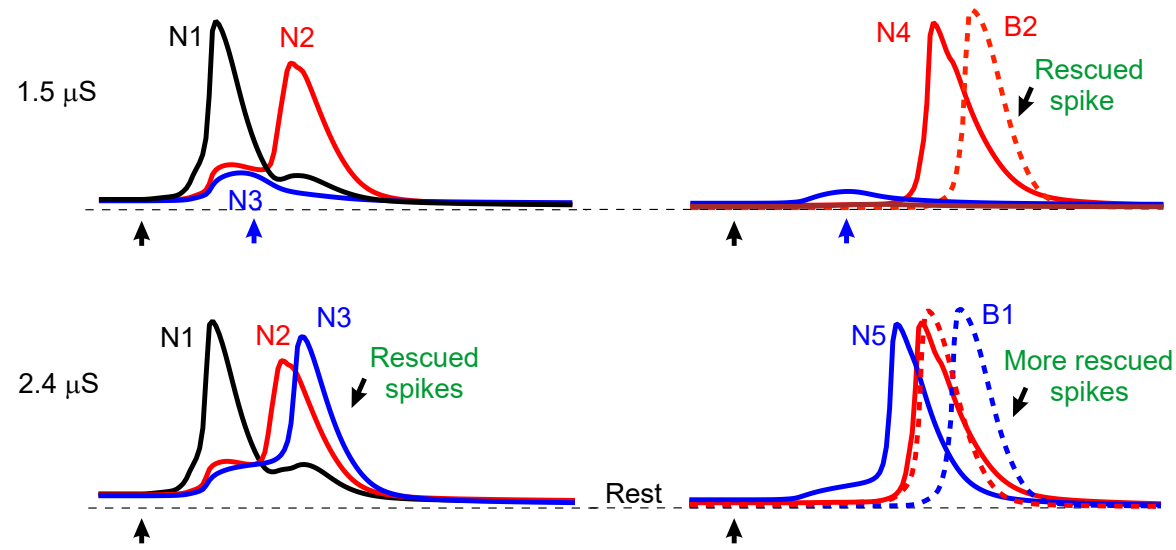

d

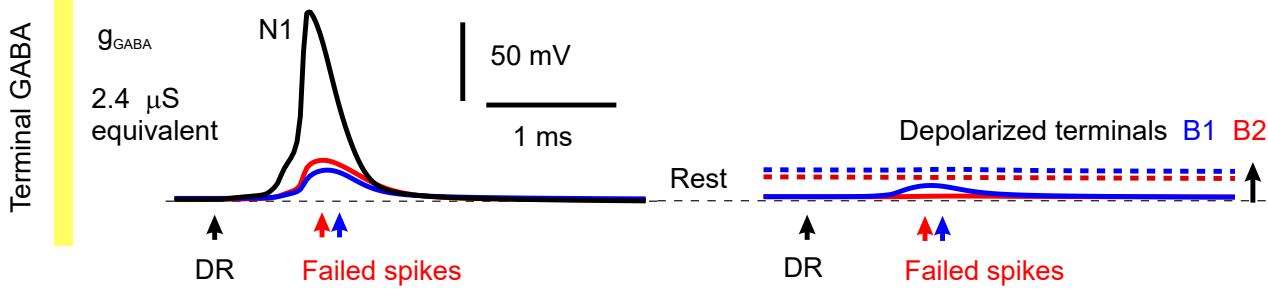

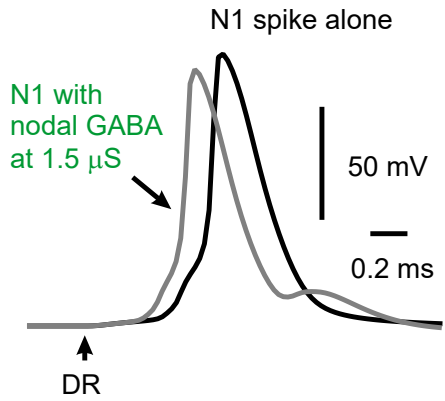

f

PAD at dorsal columns

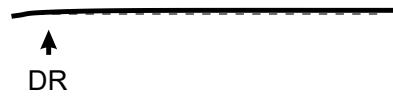

g
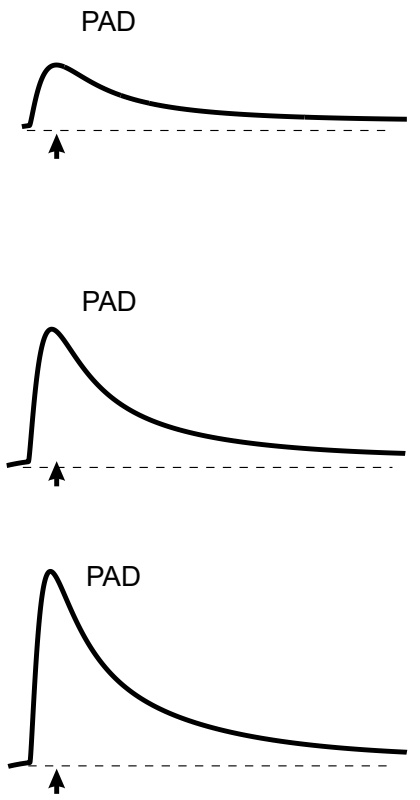

h

$$
1 \mathrm{mV} \frac{}{50 \mathrm{~ms}}
$$

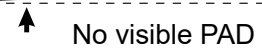


Extended Data Fig. 4 | Computer simulation of branch point failure and rescue by GABA. a, Model of a 3D reconstructed proprioceptive afferent, drawn to scale, except myelinated branch lengths all shortened an order of magnitude. Double line segments are myelinated (white) and the rest unmyelinated. Adapted from anatomical studies of Walmsley ${ }^{34}$. Nodes are indicated with a green dot, and ventrally projecting terminal boutons indicated with a yellow dot. As in our axons of Fig. 1, branch points were always at nodes. GABA $A_{A}$ receptors of equal conductance (nS) were placed at each branch point (node), and total dorsal columns (dc) depolarization from phasically activating these receptors is shown in inset. The branch lengths $(\mathrm{L})$ and computed space constants $\left(\lambda_{S}\right)$ are indicated in gray boxes for each segment of the afferent, the latter computed from subthreshold current injections into each segment. From left to right the gray boxes are for segments spanning from the dorsal columns (dc) to N0, N0 to N1, N1 to N5, N1 to N4, N4 to B2 and N5 to B1. Average space constant was $\lambda_{s}=91 \mu \mathrm{m}$, similar to in other axons ${ }^{39}$. b. Responses to simulated dorsal root (DR) stimulation (0.1 ms pulse, $2 \mathrm{nA}$, at black arrows) computed at various downstream branch points (nodes) and terminal boutons in the spinal cord, with resting potential indicated by thin dashed line $(-82 \mathrm{mV})$. A sodium spike propagated to the branch point at node $\mathrm{N} 1$, but failed to invade into the downstream branches, leaving nodes N2 and N3 with only a passive depolarization from the N1 spike (failure potential, FP). Further downstream nodes and terminals experienced vary little depolarization during this failure (N5, B1 and B2). In this case the GABA conductance was set to zero $\left(\mathrm{g}_{\mathrm{GABA}}=0\right.$, control), simulating a lack of GABA tone. Note that only the nodes beyond the parent branch at node N1 failed due to the conductance increases in large daughter branches to nodes N2 and N3 that drew more current than node N1 could provide (shunting conductances). Node 3 is particularly complex with a large unmyelinated region (black) and a nearby branch contributing to its conductance and related failure, and Node 2 has two adjacent branches contributing to its conductance. Other branch points with relatively smaller conductance increases (NO, simpler branching) did not fail to conduct spikes. Generally speaking, if the upstream node of a parent branch cannot provide enough current to activate the nodes of its daughter branches then spikes fail, and this is especially likely with multiple sequential branch points, like in N1 - N2. c, As seen experimentally, nodal $\mathrm{GABA}_{A}$ receptor activation to produce PAD prior to the DR stimulation ( $10 \mathrm{~ms}$ prior; as in Fig. 4f) rescued spikes from failing to propagate. That is, with $\mathrm{GABA}_{\mathrm{A}}$ receptors placed just at branch points (i.e., at nodes) a weak phasic activation of these receptors (conductance $g_{G A B A}=0.6-1.5 \mu \mathrm{S}$ per node shown) rescued conduction down the branch to node N2, with full nodal spikes seen at the distal node N4 and the terminal bouton B2 (DR stimulation at peak of $\mathrm{PAD}$, detailed in (g) with black arrows indicating DR stimulation timing). A larger GABA receptor activation (2.4 $\mathrm{nS}$ ) additionally rescued spike conduction down the branch to node N3, with full spike conduction to the distal node N5 and the terminal bouton B1. Note that increasing GABA conductance sped up the arrival of distal spikes (e.g. at N4 and B2), by up to $1 \mathrm{~ms}$, suggesting substantial variation in sensory transmission times induced by GABA, as we see experimentally. Also note that this nodal GABA depolarized the nodes (N1 - N3) relative to rest (thin dashed line), thus assisting spike initiation. In contrast, nodal GABA did not depolarize the terminal boutons (B1 and B2), consistent with our recent direct recordings from terminals ${ }^{16}$. Sensitivity analysis revealed similar results with a wide range of sodium channel and GABA receptor conductances, though increasing sodium conductance sufficiently prevented failure all together (not shown, but like in Extended Data Fig. 9f). d, When we removed all nodal GABA receptors and instead place them on terminal boutons (near $\mathrm{B} 1$ and $\mathrm{B} 2$, yellow, with equivalent total conductance, $2.4 \mu \mathrm{S}$ condition), then activating them did not rescue the spike propagation failure, since the associated depolarization of nodes is too attenuated at the failure point (N1-N3; no change from resting potential). The GABA receptors did depolarize the terminal boutons (B1 and B2, thick dashed lines) substantially relative to the resting potential (thin dashed lines), but this depolarization was sharply attenuated in more proximal nodes (N1-3). e, Reduction of spike height (shunt) and speeding of spike onset with increasing GABA conductance at a non-failing node (N1; model with nodal and not terminal bouton GABA conductances, c), consistent with actual recordings from axons in Fig. 3d and Extended Data Fig. 7a. f-h, PAD recorded at the dorsal columns (dc) during conditions in (b-d), respectively, as experimentally recorded dorsal PAD. A phasic GABA induced depolarization (PAD) was generated by changing GABA conductances, $\mathrm{g}_{G A B A}$, as detailed in Methods, and GABA receptor location varied as in (c-d). DRs were stimulated at the peak of this PAD in (c-d). Note that nodal (g) but not terminal (h) GABA receptors caused a visible depolarization (PAD) at the $\mathrm{dc}$, due to less electrotonic attenuation over a shorter distance to the dc. 
a

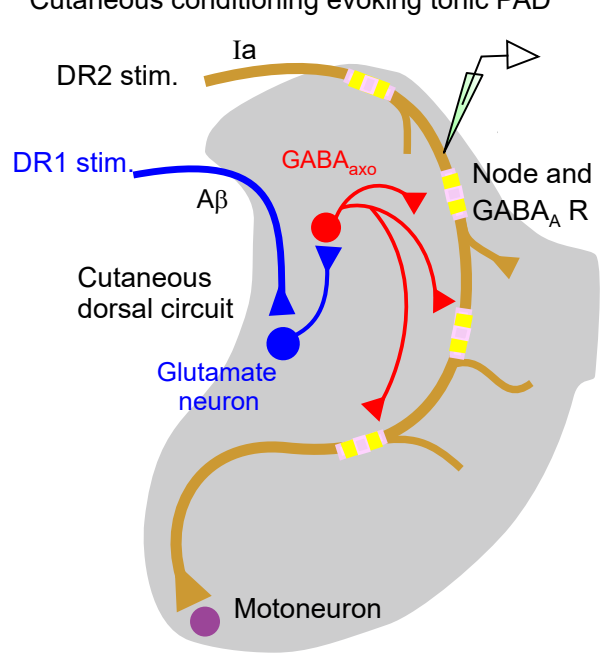

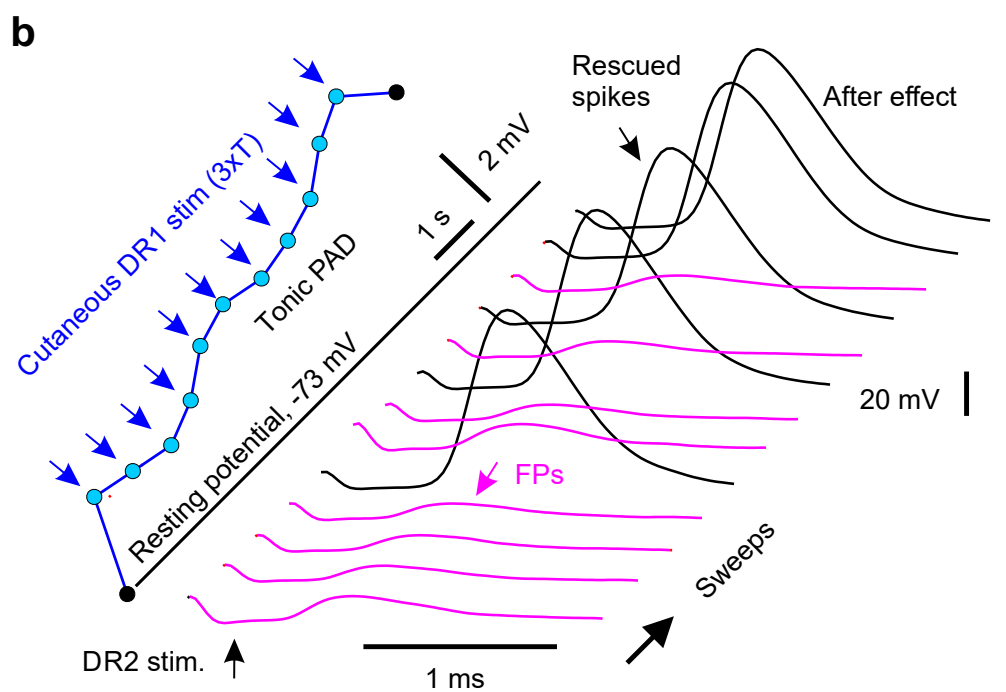

Extended Data Fig. 5 | Cutaneous driven trisynaptic circuits mediating PAD and assisting repetitive firing.

a, Cutaneous driven dorsal trisynaptic circuit mediating PAD. A minimally trisynaptic circuit is classically known to depolarize afferents via $G A B A_{a x o}$ neurons. This circuit involves sensory afferents activating excitatory intermediary neurons (glutamatergic) that in turn activate $\mathrm{GABA}_{\text {axo }}$ neurons that return to innervate sensory axons ${ }^{14}$. Even though $\mathrm{GABA}_{\text {axo }}$ neurons are small ${ }^{6}$ this circuit influences afferents over widespread regions of the spinal cord ${ }^{16}$. Specifically, the activation of a small group of sensory axons in just one DR or nerve causes this trisynaptic circuit to produce a widespread activation of many axons across the spinal cord, even many segments away and across the midline ${ }^{16}$. This allowed us to activate PAD from adjacent roots without directly activating an axon in a particular root, as detailed in Fig. 4 and the rest of this figure. One variant of this classic trisynaptic circuit specifically involves cutaneous stimulation activating dorsal intermediary neurons ${ }^{14,15}$ that activates $\mathrm{GABA}_{a x o}$ neurons that in turn innervate cutaneous afferents, which we term the cutaneous dorsal circuit. While this cutaneous dorsal circuit also synaptically innervates some proprioceptive afferents ${ }^{113}(\mathbf{a})$, its main action on proprioceptive afferents is to produce a pronounced extrasynaptic spillover of GABA that depolarizes these afferents tonically via $\alpha 5 \mathrm{GABA}_{A}$ receptors (termed: tonic PAD, $L 655708$ sensitive), especially with repetitive cutaneous nerve stimulation $\left(1-200 \mathrm{~Hz}\right.$ ) that leads to minutes of depolarization ${ }^{16}$, and we see similar tonic PAD here (detailed next). b, Intracellular recording from a proprioceptive axon branch in rat dorsal horn (sacral S4 axon, DR2). The axon branch spontaneously exhibited spike propagation failure when its was stimulated alone (denoted DR2 stimulation, repeated at 1 $\mathrm{Hz}, 1.1 \times \mathrm{T}, 0.1 \mathrm{~ms}$ ), with only a small failure potential (FP) visible (lower pink traces). Activation of a largely cutaneous DR (caudal Ca1 DR, innervating the tip of the tail, stimulation at intensity for cutaneous afferents, 3xT, 0.1 ms; denoted DR1) evoked a slowly rising tonic PAD when repeated at $1 \mathrm{~Hz}$ (blue). When the axon stimulation (DR2 stimulation) was combined with the repeated cutaneous stimulation (DR1, 60 ms prior to each DR2 stimulation) the slowly building PAD prevented spike failure (black spikes), and this outlasted the cutaneous stimulation (after effect). Similar results obtained in $n=20 / 20$ axons tested. 
a Proprioceptive conditioning evoking PAD (self-facilitation)
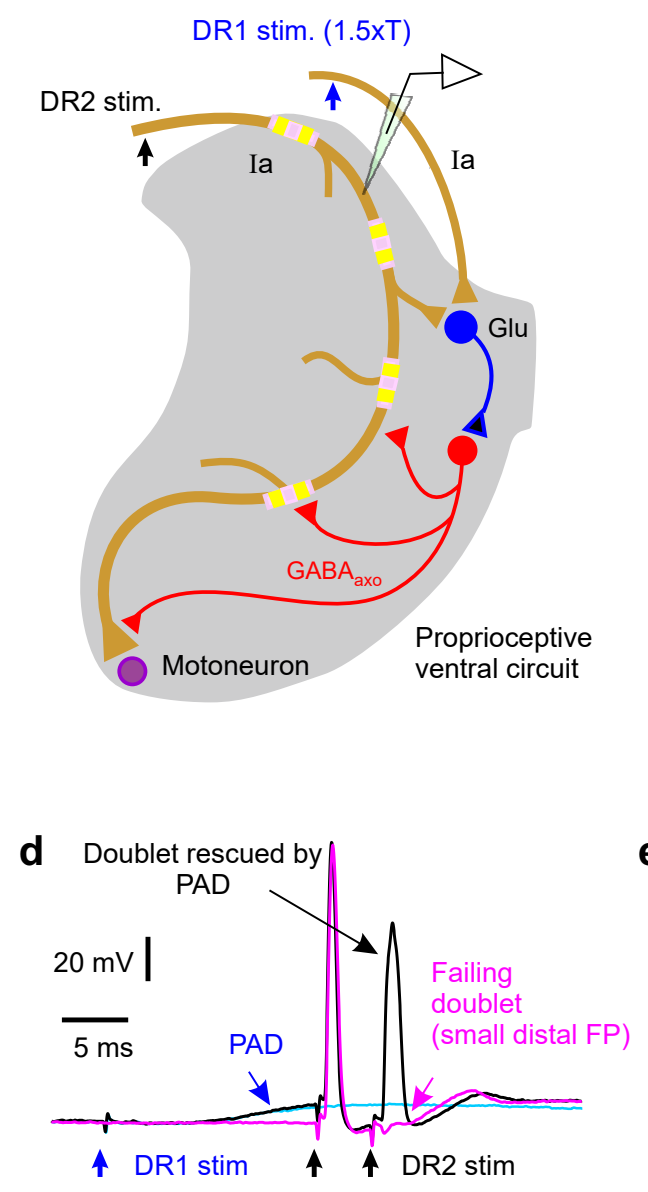

b
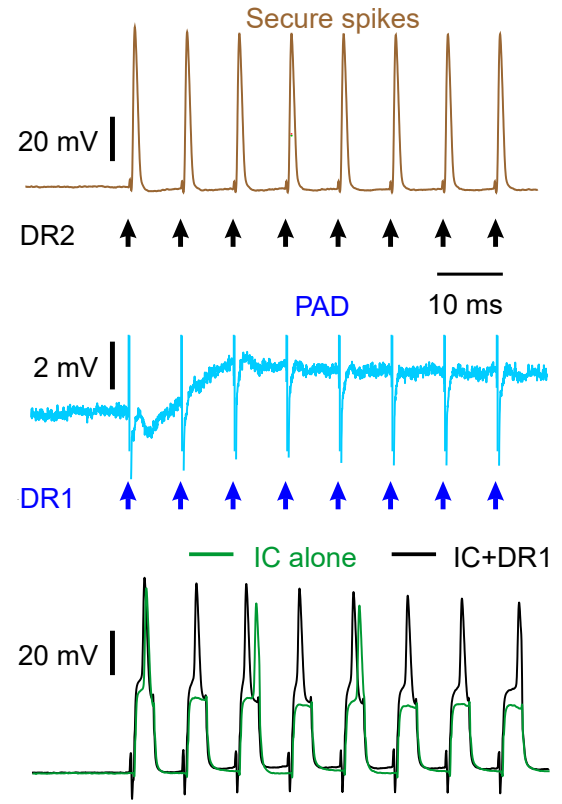

2 nA $\mid$ Current injection (IC)

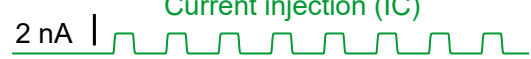

C

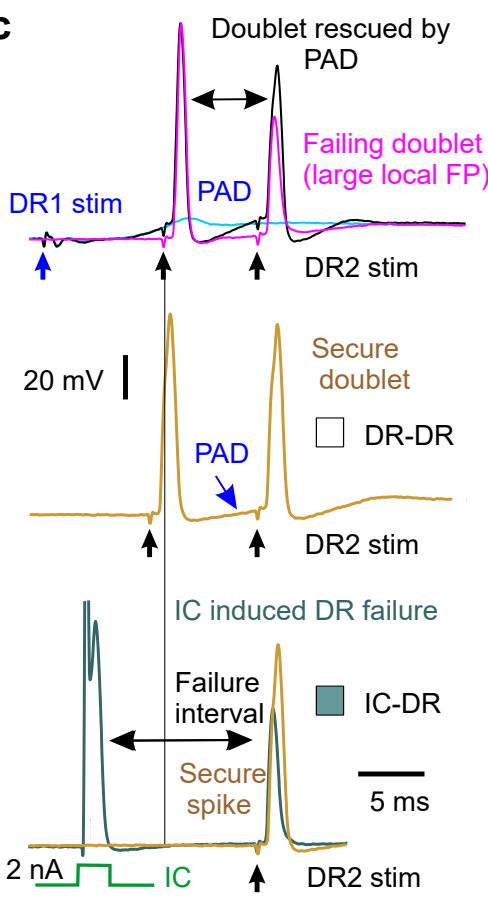

e Minumum interval (maximal rate) f Failures from repetition rescued by PAD for repetitive firin
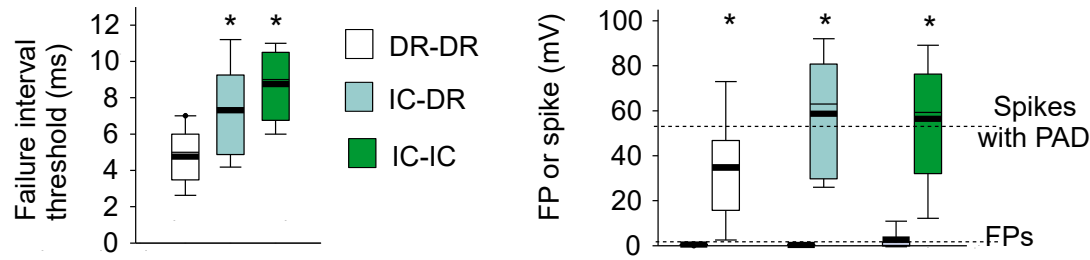
Extended Data Fig. 6 | Proprioceptive driven trisynaptic circuit for PAD enabling high frequency spike transmission. a, Proprioceptive driven ventral trisynaptic circuit mediating PAD. Another variant of the classic trisynaptic circuit involves proprioceptive afferents activating excitatory intermediary neurons (glutamatergic) that then activate GABA $_{a x o}$ neurons that innervate these same afferents, including ventral terminal regions of the afferents ${ }^{14,16}$. This circuit is more ventrally located compared to the cutaneous dorsal PAD circuit ${ }^{14}$, and thus, we term it the proprioceptive ventral PAD circuit. Importantly, the circuit is recurrent, with proprioceptive afferents causing self-facilitation of themselves (via homonymous PAD). It produces a fast phasic axon depolarization (phasic PAD, fast synaptic; Fig. 4c), as well as a slower tonic depolarization (tonic PAD, Fig. 4c, likely from extrasynaptic GABA spillover), as detailed previously ${ }^{16}$. Since proprioceptive sensory axons naturally fire at high rates ${ }^{114}$ where they are vulnerable to spike failure (Fig. 2e-f), we examined the action of self-facilitation by GABA on this failure. b-f, During rapid repetitive stimulation of a DR to evoke spikes in a proprioceptive axon there was an inevitable activation of PAD from low threshold proprioceptive axons (homonymous PAD, b). This PAD helped spikes fire at high physiological rates of up to $200-300 \mathrm{~Hz}(5-3 \mathrm{~ms}$ intervals) before spike inactivation and failure occurred because, in absence of PAD, isolated repetitive activation of the axon with intracellular current pulses (IC) led to failure at much lower rates ( $100 \mathrm{~Hz}$; longer spike intervals; b, e), even after just two stimuli (doublets; c, e). Additional PAD evoked by simultaneous stimulation of an adjacent DR (2xT) reduced failure from fast repeated IC stimuli (b, $\mathbf{f})$, repeated DR stimuli (doublet, $\mathbf{c}, \mathbf{d}, \mathbf{f}$ ) or hybrid IC-DR stimulation pairs (f). Legend details are as follows: b, Intracellular recording from proprioceptive axon in rat dorsal horn (sacral S4 axon) with spikes securely evoked by fast repeated DR stimulation (top, $1.1 \times \mathrm{T}, 0.1 \mathrm{~ms}$, sacral S4 DR, denoted DR2; resting potential $-68 \mathrm{mV}$ ), but spikes failing intermittently with repeated intracellular current injection (IC) at the same rate (bottom green), due to sodium channel inactivation. The reason that spike failure does not occur with the fast DR stimulation is that it is accompanied by a build up of tonic PAD (from self-activation) that helps prevent failure, because adding to the IC stimulation a simultaneous conditioning stimulation of other proprioceptive afferents in an adjacent DR (DR1 stim at 1.5xT, $0.1 \mathrm{~ms}$, S3 DR) prevents spike failure (black trace, bottom), via the proprioceptive ventral circuit (a). This DR1 conditioning stimulation does not directly activate spikes in the axon, but it causes a fast depolarization (phasic PAD) that rapidly helps spikes (as early as 6 - 10 ms later), and a building tonic depolarization (tonic PAD) with repetition that further helps later spikes in the stimulation train (DR1 stimulation alone blue, middle trace). Similar results obtained in $n=7 / 7$ axons. Likely similar tonic PAD and associated increased spike conduction helps explain post-tetanic potentiation of the monosynaptic EPSP, as previously suggested ${ }^{29}$. c, Repeated DR stimulation at higher rates eventually causes spike failure in proprioceptive axons (sodium spike relatively refractory), as shown in the top panel where a double stimulation (doublet, S4 DR, denoted DR2-DR2, 1.1xT, 0.1 ms, resting at $-75 \mathrm{mV}$ ) exhibits failure on the second spike (with large FP indicated, magenta). However, additional PAD provided by stimulating an adjacent DR (DR1; 1.5xT, group I intensity, $0.1 \mathrm{~ms}$ ) about $10 \mathrm{~ms}$ earlier helps prevent this spike failure (black trace; blue trace: PAD alone). When the same axon was stimulated slightly slower (with a longer doublet interval, second plot, DR-DR) failure did not occur, which we designate the failure interval threshold, which is quantified in (e). The selfactivated PAD caused by the first DR stimulation in this doublet helped prevent failure in second DR stimulation because replacing the first DR stimulation with an intracellular stimulation (IC, $2 \mathrm{nA}$ ) to activate the spike leads to failure of the second spike evoked by the DR stimulation at much longer intervals (lower trace, IC-DR). d, Another example of a failed doublet spike (DR2-DR2 stim, 1.1xT, $0.1 \mathrm{~ms}$ ) that was rescued by PAD evoked by adjacent DR stimulation (DR1 1.5xT, 0.1 $\mathrm{ms}$, resting at $-78 \mathrm{mV}$ ), as in the top plots of $(\mathbf{c})$, except that in this case the failure is at a more distal node, since the FP is small. e, Failure interval threshold (minimum firing interval prior to failure, or maximal firing rate) with DR doublets (DRDR), IC doublets (IC-IC) or IC-DR pair stimulation. Note the shorter intervals possible with the PAD evoked by the first stimulation (DR-DR). * significantly longer than minimum DR doublet interval (DR-DR), $n=18$ each, $P<0.05$. f, Quantification of the FP heights that were induced by a fast doublet (DR-DR or IC-IC; $n=14$ each, at failure threshold interval) or IC and DR stimulation (IC-DR; $n=10$ ), and the rescue of spikes by PAD evoked by adjacent DR stimulation. * significant increase in height with $P A D, P<0.05$. 


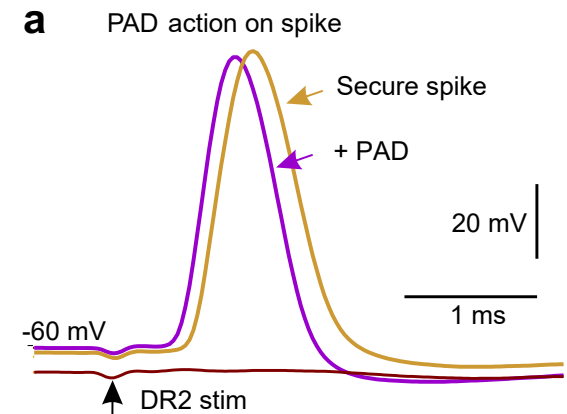

b

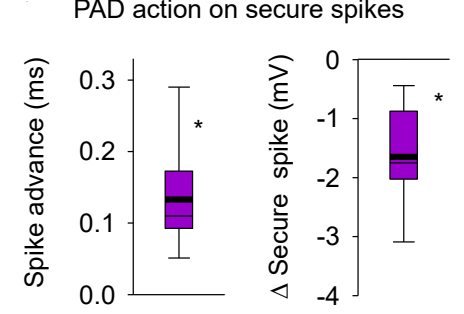

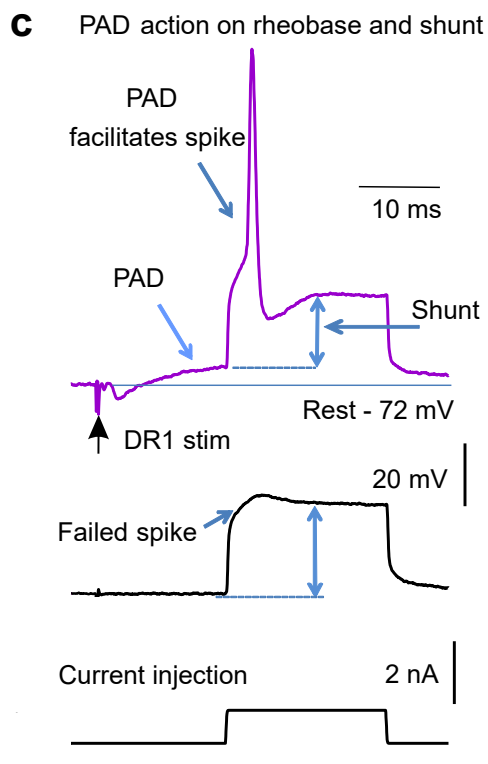
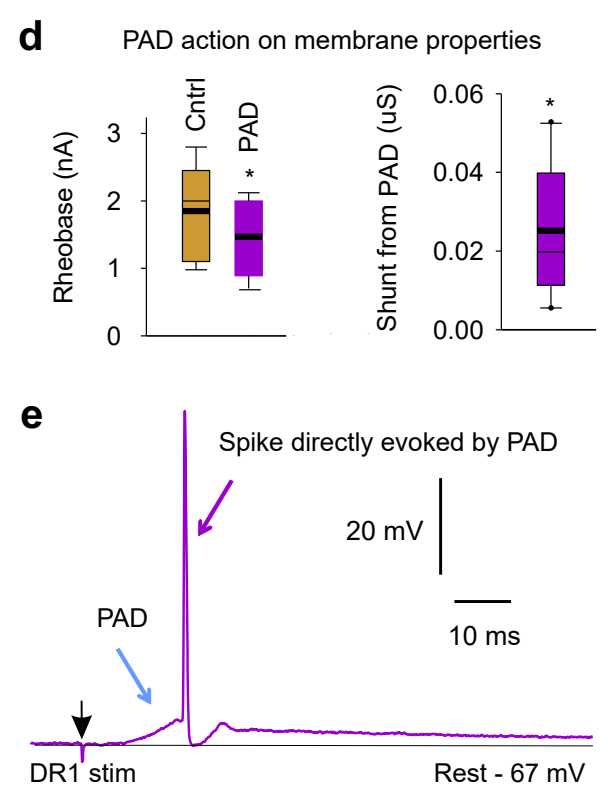

Extended Data Fig. 7 | Other excitatory actions of $\mathrm{GABA}_{\mathrm{A}}$ receptors on proprioceptive axons.

a, Intracellular recording from a sacral S3 proprioceptive la afferent branch in the dorsal horn with a secure spike evoked by S3 DR stimulation at rest (DR2, 1.1xT, $0.1 \mathrm{~ms},-60 \mathrm{mV}$ rest, rat). Sensory-evoked PAD initiated by stimulating an adjacent DR (DR1; S4 DR; 2xT, $0.1 \mathrm{~ms}$ pulse, as in Fig. 4) $10 \mathrm{~ms}$ prior to the DR2 stimulation only moderately influenced the spike (DR1 stimulation time not shown). It sped up the spike latency and rise time, reduced the fall time and slightly reduced the spike height. Hyperpolarization induced spike failure (lower trace), as in Extend Data Fig. 2a. b, Summary box plots of change in spike peak latency (advance) and height with prior sensory PAD activation as in (a). *significant change, $P<0.05$, $n=20$ and 26, respectively. c, Intracellular recording from an S3 proprioceptive la afferent branch in the dorsal horn with a brief current injection pulse just subthreshold to initiating a spike (near rheobase, bottom trace) at rest, only initiating a passive response with a small failed spike (middle trace). However, prior activation of PAD by stimulating an adjacent DR (DR1 as in A; S4 DR, 2xT, 0.1ms top trace) allowed the same current pulse to evoke a spike (above rheobase). The passive response to the current injection (double blue arrow; resistance $R=V / I$ ) was decreased during the $P A D$, corresponding to an increase in conductance, that contributed to a shunt (reduction) of the currents generating the spike, though this only caused about a $1 \%$ drop in spike height $(1 \mathrm{mV} ; \mathbf{b})$. DCC recording mode, as in Extend Data Fig. 2a-d. d, Summary box plots of rheobase (current threshold from c) before and during PAD, and change in shunt (conductance = 1/R) with PAD, as in (c). * significant change with PAD, $P<0.05, n=37$ axon branches. $e$, By itself sensory evoked PAD sometimes initiated a spike on its rising phase, when the DR stimulation was large enough, demonstrating a direct excitatory action of GABA receptors, as previously reported ${ }^{16}$. These spikes propagate antidromically toward the DR; and are thus termed dorsal root reflexes (DRR). Example intracellular recording from rat sacral S3 proprioceptive afferent branch in the dorsal horn, similar to in Fig. 4e (top trace), but PAD evoked by a larger DR1 stimulation (S4 DR stimulation 3.5xT, $0.1 \mathrm{~ms}$ ) that itself evoked a spike (DRR). These PAD evoked spikes occur with a variable latency of $10-30 \mathrm{~ms}^{16}$ and thus make axons refractory for about $30 \mathrm{~ms}$ after the DR stimulation ${ }^{115}$. These spikes can also evoke EPSPs in motoneurons via the monosynaptic pathway ${ }^{9}$, and thus also produce a post activation depression of the EPSPs for many seconds. We thus kept the PAD low when examining the effects of PAD on EPSPs, to avoid these spikes and their subsequent inhibitory action (in Figs. $5-7$ ). 
a

Ventral horn microstimulation $(\mathrm{VH})$

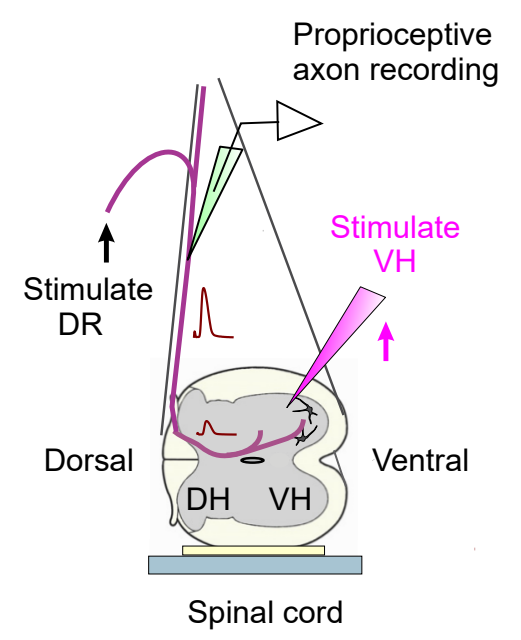

b Threshold for $\mathrm{VH}$ doublet: $T_{\mathrm{VH} 2}$

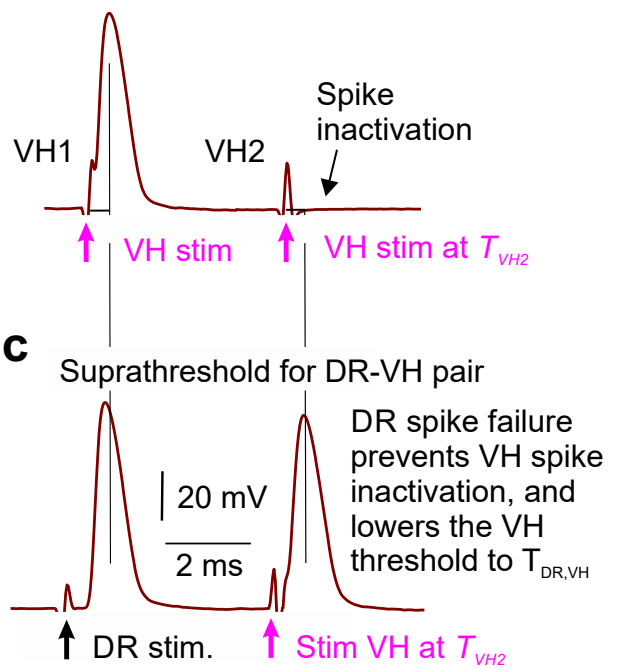

d Spike failure calculation

$$
\begin{gathered}
\% \text { Failure }=\frac{\left(T_{V H 2}-T_{D R, V H}\right)}{\left(T_{V H 2}-T_{V H 1}\right)} \times 100 \\
100 \% \text { failure: } T_{D R, V H}=T_{V H 1} \\
0 \% \text { failure: } T_{D R, V H}=T_{V H 2}
\end{gathered}
$$

e Spike propagation failure

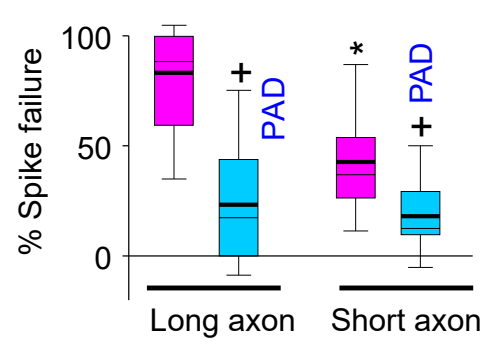

Extended Data Fig. 8 | Estimating the overall spike conduction failure from the dorsal root to the motoneurons. a, Experimental setup to indirectly measure sensory axon conduction failure following DR stimulation, by examining whether failed axon segments are relatively less refractory to activation after failure, using a double pulse method adapted from Wall ${ }^{30}$. A tungsten microelectrode $(12 \mathrm{M} \Omega$ ) was placed in the ventral horn $(\mathrm{VH})$ near the sensory axon terminals on motoneurons ( $\mathrm{S} 3$ or $\mathrm{S} 4 \mathrm{VH}$ ), to activate the branches/nodes of the axon projecting to the motoneuron that may have failed (VH stimulation). Spikes from VH or DR stimulation were recorded intracellularly in a proprioceptive axon penetrated in the dorsal columns. b, VH threshold in refractory period. Rapidly repeated VH stimulation (VH doublet; two 0.1 ms pulses) at an interval short enough to produce spike inactivation on the second stimulation (4 ms), with stimulus current adjusted to threshold for inactivation, $\mathrm{T}_{\mathrm{VH} 2}$. This $\mathrm{T}_{\mathrm{VH} 2}(\sim 15 \mathrm{uA})$ was always higher than the threshold $\mathrm{VH}$ stimulation for evoking a spike with the first stimulation, $\mathrm{T}_{\mathrm{VH} 1}(\sim 10 \mathrm{uA}$, not shown). Recorded in sacral $\mathrm{S} 4$ afferent resting at $-72 \mathrm{mV}$, with doublets repeated at $3 \mathrm{~s}$ intervals to determine current thresholds. c, $\mathrm{VH}$ threshold after DR stimulation. Similar repeated activation of the axon in (b), but with the first activation from a DR stimulation (at 1.5x DR threshold) and the second from VH stimulation at the $\mathrm{T}_{\mathrm{VH} 2}$ intensity from (b). In this case the $\mathrm{VH}$ stimulation readily activated the axon spike, likely because the DR-evoked spike did not propagate to the $\mathrm{VH}$, leaving the silent portion of the axon non refractory. Thus, this $\mathrm{VH}$ stimulation evoked spikes with a lower current than $\mathrm{T}_{V H 2}$, with this lower threshold denoted $\mathrm{T}_{\mathrm{DR}, \mathrm{VH}}(\sim 12 \mu \mathrm{A}$, not shown). This DR - VH stimulation interval was deliberately set too short for the involvement of PAD (which rises in $>4 \mathrm{~ms}$; Fig. 4). d, Computation of spike failure based on changes in $\mathrm{VH}$ stimulation thresholds. If the DR-evoked spike entirely fails to propagate to the $\mathrm{VH}$, then the threshold for subsequently activating the $\mathrm{VH}\left(\mathrm{T}_{\mathrm{DR}, \mathrm{VH}}\right)$ should be the same as the threshold without any prior activation $\left(T_{V H 1}=T_{D R, V H}\right)$, whereas if it does not fail then the threshold for activating the $\mathrm{VH}$ should be the same as with a $\mathrm{VH}$ doublet $\left(\mathrm{T}_{\mathrm{VH} 2}=\mathrm{T}_{\mathrm{DR}, \mathrm{VH}}\right)$. In between these two extreme scenarios, the $\mathrm{DR}$ evoked spike may only partially fail to propagate spikes to the $\mathrm{VH}$; in this case $\mathrm{T}_{\mathrm{DR}, \mathrm{VH}}$ should be between $\mathrm{T}_{\mathrm{VH} 1}$ and $\mathrm{T}_{\mathrm{VH} 2}$, with the difference $\mathrm{T}_{\mathrm{VH} 2}-\mathrm{T}_{\mathrm{VH} 1}$ representing the range of possible thresholds between full failure and conduction. Overall the \% conduction failure can be thus quantified as: $\left(\mathrm{T}_{\mathrm{VH} 2}-\mathrm{T}_{\mathrm{DR}, \mathrm{VH}}\right) /\left(\mathrm{T}_{\mathrm{VH} 2}-\mathrm{T}_{\mathrm{VH} 1}\right) * 100 \%$, which is $100 \%$ at full failure and $0 \%$ with no failure. e, Average spike conduction failure to the $\mathrm{VH}$ in proprioceptive axons, and decrease following a DR conditioning stimulation that depolarized the axon (PAD). Box plots of failure estimated as in (b-d). Prior DR conditioning to produce PAD (via adjacent S4 or Ca1 DR stimulation at 3XT) reduced the failure estimated 20 ms later by the paired-pulse conduction testing (repeating DR - VH stimulations of $\mathbf{b}-\mathbf{d}$ ). DR conditioning itself lowered the thresholds for VH activation, as previously reported (not shown) ${ }^{31}$. We studied two lengths of axons: long axons (intersegmental, $n=10$ ) with the VH stimulation one segment away from the recording site, and short axons (segmental, $n=12$ ) with the VH stimulation near the recording site, in the same segment. + significantly less failure with PAD and * significantly less failure with short compared to long axons, $P<0.05$. 
a Extracellular recording

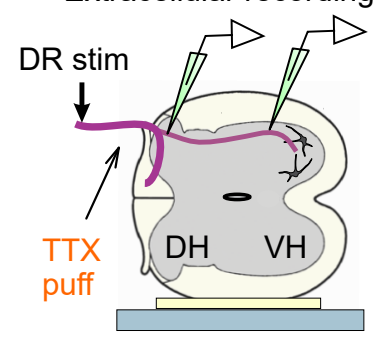

b Ventral horn

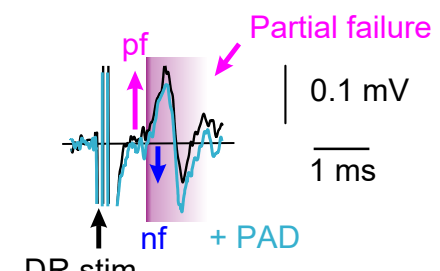

DR stim

g Conduction calculation

Conduction index $=\frac{n f}{(n f+p f)} \times 100$

$p f=$ positive field amplitude

$n f=$ negative field amplitude

100\% conduction: $p f=0$

$0 \%$ conduction: $n f=0$
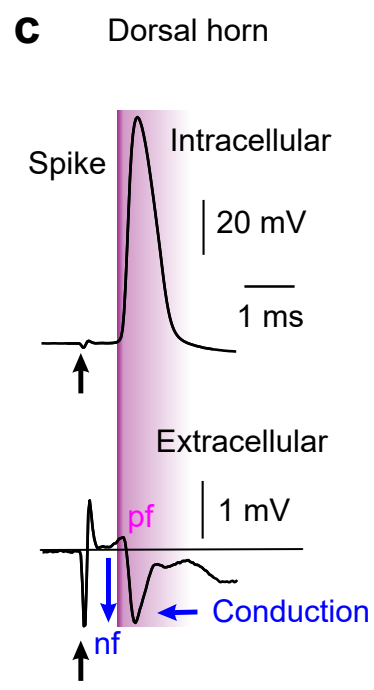

DR stim d Dorsal horn

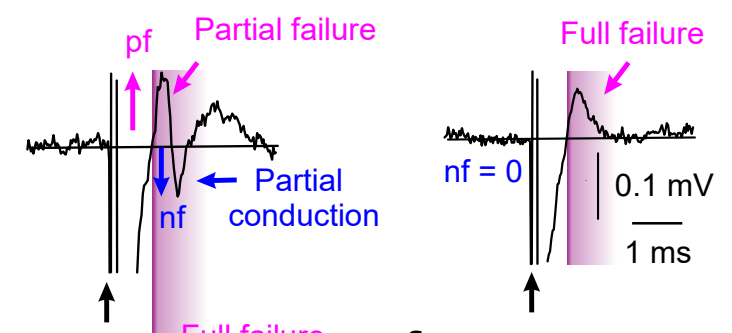

Full failure

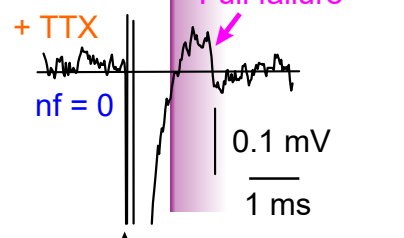

$\uparrow$ DR stim f Ventral horn - $\mathrm{Mg}++$

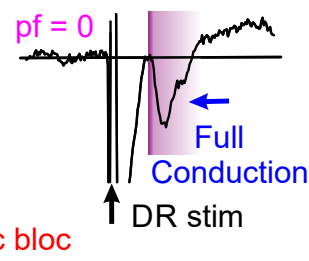

h Dorsal horn

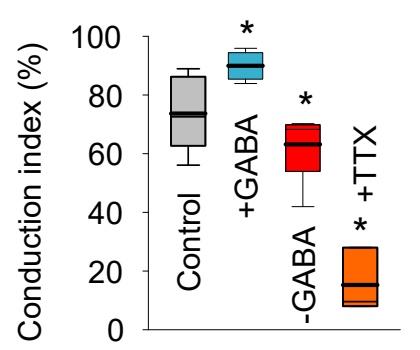

Ventral horn

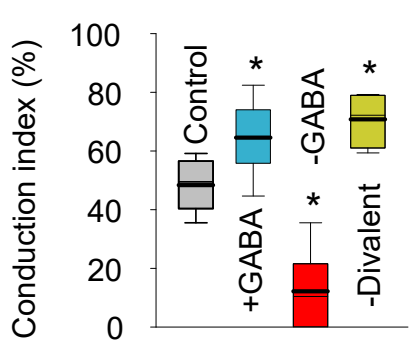


Extended Data Fig. 9 | Axonal spike conduction estimated from extracellular recordings.

a, Experimental setup to directly record spike conduction failure in proprioceptive axon terminals in the ventral horn (VH) following DR stimulation. Extracellular (EC) recordings from axon terminals in $\mathrm{VH}$, with glass electrode positioned just outside these axons, and for comparison EC recording in the dorsal horn (DH). b, EC field recorded in VH after DR stimulation (S4 DR, 1.1xT), with a relatively large initial positive field (magenta arrows, pf) resulting from passively conducted axial current from sodium spikes at distant nodes (closer to the DR; outward current at electrode), some of which fail to propagate spikes to the $\mathrm{VH}$ recording site; thus, this field is a measure of conduction failure, as demonstrated in (c-f) below. Following this, a negative field arises (blue arrow, nf), resulting from spikes arising at nodes near the electrode (inward current); thus, this field is a measure of secure conduction. Reducing conduction failure by depolarizing the axon (+PAD) with a prior conditioning stimulation of an adjacent DR (Ca1, 2xT, 30 ms prior), decreased the positive field (pf) and increased the negative field ( $\mathrm{nf}$ ), consistent with increased conduction to the terminals, and in retrospect the same as Sypert et al. (1980) saw in cat (their Fig. 4). Large stimulus artifacts prior to these fields are truncated. c, Control recordings from proprioceptive axons in dorsal horn (DH) to confirm the relation of the EC negative field (nf) to spike conduction. Intracellular (IC) recording from axon (sacral S4, resting at $-64 \mathrm{mV}$ ) and EC recording just outside the same axon, showing the DR evoked spike (IC) arriving at about the time of the negative EC field (nf). There is likely little spike failure in this axon or nearby axons, due to the very small initial positive field (pf). EC fields are larger in $\mathrm{DH}$ compared to VH $(G, 10 x)$, and thus the artifact is relatively smaller. $d$, Locally blocking nodes with TTX to confirm the relation of the positive EC field to spike failure. EC recording from proprioceptive axon in the dorsal horn (S4), with an initial positive field (pf) followed by a negative field (nf), indicative of mixed failure and conduction. A local puff of TTX (10 $\mu$ of $100 \mu \mathrm{M})$ on the DR just adjacent to the recording site to transiently block DR conduction eliminated the negative field (nf) and broadened the positive field (pf), consistent with distal nodes upstream of the TTX block generating the positive field via passive axial current conduction, and closer nodes not spiking. Recordings were in the presence of synaptic blockade (with glutamate receptor blockers, kynurenic acid, CNQX and APV, at doses of 1000, 100 and $50 \mu \mathrm{M}$ respectively), to prevent TTX spillover

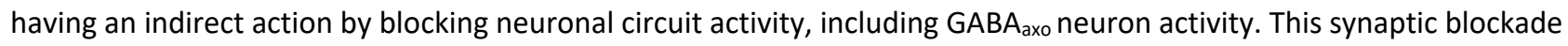
itself contributed to some spike failure, consistent with a block of GABA axo neuron activity, as there was a more prominent positive field (pf) compared to without blockade in (c). e, EC field recorded from terminals of proprioceptive axons in the ventral horn near motoneurons (S4), in the presence of an excitatory synaptic block that largely eliminates most neuronal circuit behaviour (with kynurenic acid, CNQX and APV, as in d). In this synaptic block negative fields were generally absent $(\mathrm{nf}=0$ ), and only prominent positive fields (pf) occurred (as with TTX block), suggesting that conduction to the VH often completely failed when circuit behavior is blocked, which likely indirectly reduces GABA $A_{a x o}$ neuronal circuit activity and its associated facilitation of nodal conduction. $\mathbf{f}$, Rescue of spike conduction to the ventral horn by increasing sodium channel excitability by reducing the divalent cations $\mathrm{Mg}++$ and $\mathrm{Ca}++$ in the bath medium ${ }^{75}$. Same EC field recording as in e, but with divalent cations reduced $\left(\mathrm{Mg}^{++}, 0 \mathrm{mM} ; \mathrm{Ca}^{++}, 0.1 \mathrm{mM}\right)$. The positive field was largely eliminated $(\mathrm{pf}=0)$ and replaced by a negative field (nf), consistent with elimination of conduction failure, and proving that the positive field is not a trivial property of axon terminals ${ }^{73,74,116} \cdot \mathbf{g}$, Conduction index computed from positive (pf) and negative (nf) field amplitudes as: $\mathrm{nf} /(\mathrm{nf}+\mathrm{pf}) \times 100 \%$, which approaches $100 \%$ for full conduction ( $\mathrm{pf} \sim 0$; as in c) and $0 \%$ for no conduction ( $\mathrm{nf}=0$; as in e). $\mathbf{h}-\mathbf{i}$, Summary of conduction index estimated from EC field potentials, shown with box plots. Without drugs present in the recording chamber, the axon conduction from the DR to the dorsal horn is about is $70 \%(\mathbf{h}, n=17)$, consistent with Fig. 2 , whereas conduction from the DR to the $\mathrm{VH}$ is only about $50 \%(\mathbf{i}, n=9)$, suggesting substantial failure at the many branch

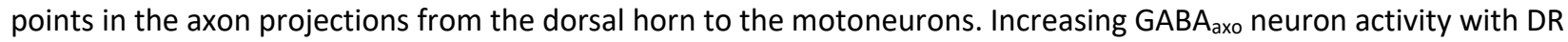
conditioning ( $30-60$ ms prior) increased conduction (+GABA, in both the $\mathrm{DH}$ and $\mathrm{VH}, n=5$ and 9 , as in b), whereas decreasing GABA and all circuit activity in a synaptic blockade decreased conduction (-GABA, in both $\mathrm{DH}$ and $\mathrm{VH}, n=5$ and 6 , as in d-e). TTX $(n=4, \mathrm{~h})$ or removal of divalent cations $\left(\mathrm{Mg}^{++}\right.$and $\mathrm{Ca}^{++}$, -Divalent, $\left.n=4, \mathrm{i}\right)$ reduced or increased conduction, respectively (as in $\mathbf{d}$ and $\mathbf{f}$ ). ${ }^{*}$ significant difference from control pre-drug or pre-conditioning, $P<0.05$. 
a

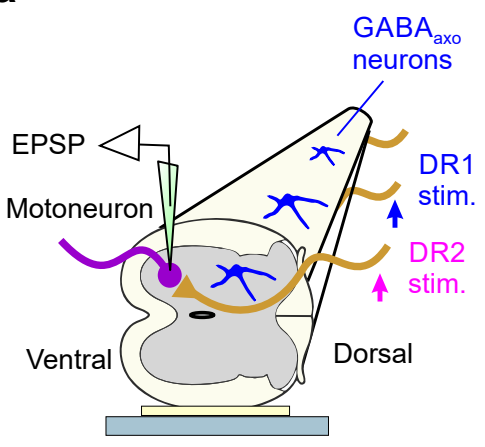

Spinal cord b

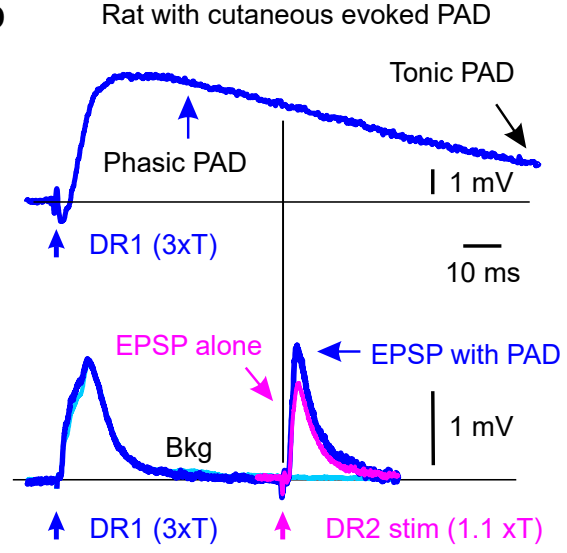

C

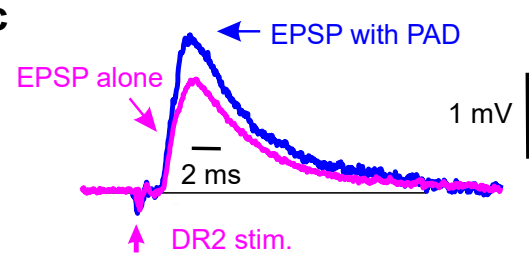

d

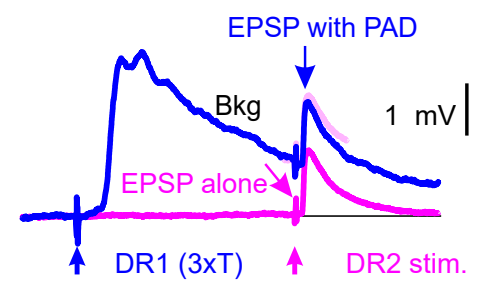

e

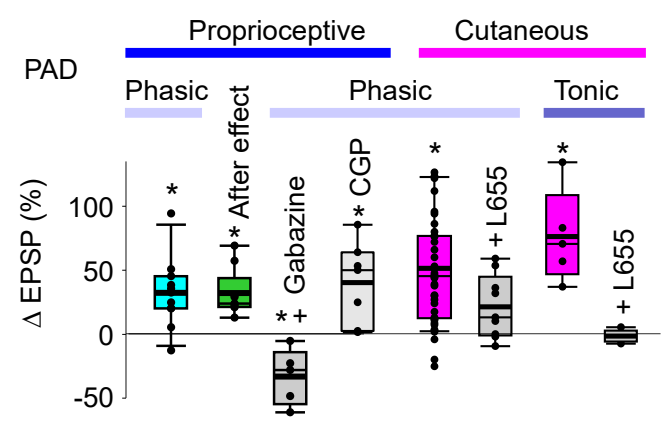

f Varying EPSPs

$1.1 \mathrm{xT} 1.5 \mathrm{x}$

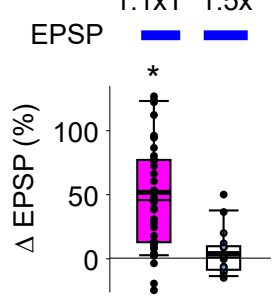

9

Conditioning raising Bkg

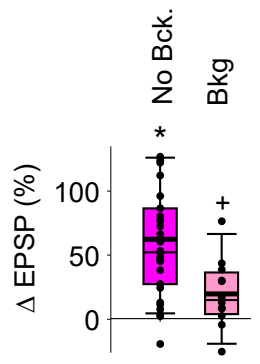

h Postsynaptic action without depolarization

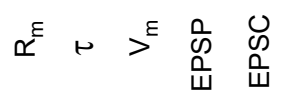

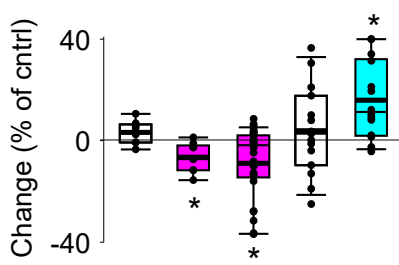


Extended Data Fig. 10 | Sensory evoked facilitation of monosynaptic EPSPs by GABA receptors.

a, Whole spinal cord ex vivo preparation for intracellular recording of EPSPs from motoneurons while stimulating dorsal roots (DRs). b, Monosynaptic EPSP in an S4 motoneuron evoked by a proprioceptive group I stimulation of the S4 DR (1.1xT, denoted DR2, lower traces; resting potential -75 mV: black line), alone (pink) and $60 \mathrm{~ms}$ after (blue) a conditioning stimulation of cutaneous afferents in rat to evoked PAD (stimulation of the largely cutaneous Ca1 DR, 2.5xT; denoted DR1). Averages of 10 trials each at $10 \mathrm{~s}$ intervals. PAD evoked by the same cutaneous conditioning stimulation in a proprioceptive S4 DR afferent is shown for reference (top, recorded separately, as in Fig. 4b). c, EPSPs from (b) on expanded time scale. d, Similar to (b), but stronger conditioning stimulation (DR1, 3xT) evoking background postsynaptic activity (blue, Bkg) that lasted longer than $60 \mathrm{~ms}$, and slightly inhibited the EPSP, likely from increased postsynaptic conductances shunting the EPSP (postsynaptic inhibition; light pink: overlay of EPSP alone) and masking nodal facilitation. e, Summary box plots of facilitation of EPSPs during phasic PAD evoked by either proprioceptive conditioning (S3 or contralateral S4 DR stimulation, $1.1 \times \mathrm{T}$, in rats and mice similar and combined, $n=6$ and 5, blue) or cutaneous conditioning (Ca1 DR stimulation, 2-3xT, in rats, $n=42$, pink), and action of $\mathrm{GABA}_{\mathrm{A}}$ and $\mathrm{GABA}_{B}$ antagonists (gabazine $50 \mu \mathrm{M}$, L655708 $0.3 \mu \mathrm{M}$ and CGP55845 $0.3 \mu \mathrm{M}$, grey, $n=5-9$ each). EPSPs evoked in S3 and S4 motoneurons by DR2 (S3 or S4) stimulation at 1.1T, as in (b). Facilitation measured 60 ms post conditioning during phasic PAD (phasic condition indicated) and when postsynaptic actions of conditioning ( $\mathrm{Bkg}$ ) were minimal (as in b). After conditioning was completed EPSP testing continued and revealed a residual facilitation that lasted for 10 - $100 \mathrm{~s}$ (After effect, green, $n=9$ tested), due to a build up of tonic PAD, after which the EPSP returned to baseline (not shown), similar to post-tetanic potentiation ${ }^{29}$. Also, a brief high frequency cutaneous stimulation train $(200 \mathrm{~Hz}, 0.5 \mathrm{~s}, 2.5 \mathrm{xT})$ that led to a very long lasting depolarization of proprioceptive axons (Tonic PAD, example in Fig. $5 \mathrm{~g}$ ) caused a facilitation of the monosynaptic EPSP that lasted for minutes (average shown, tonic cutaneous condition), and this was blocked by $L 655708$ (in rats, $n=5$ each). ${ }^{*} P<0.05$ : significant change with conditioning. $+P<0.05$ : significant change with antagonist. Raw data points shown to indicate occasional inhibition of the MSR by conditioning, but overall facilitation. ChR2 activation of GABAaxo neurons lacked these long tonic PAD-mediated after effects on the EPSP facilitation (Fig. 5e-f, Post), suggesting an additional source of GABA mediating after effects. $f$, Summary box plots of change in EPSP induced by cutaneous DR (DR1) conditioning (and associated phasic PAD) 60 ms prior to evoking the EPSP, with varying EPSP stimulation intensity. When the DR that evoked the test EPSP (DR2) was stimulated at an intensity that produced less than half the maximal EPSP height (1.1xT, 30\% max EPSP, $n=42$, same data as in e) the facilitation of EPSP by conditioning was larger than when this DR2 stimulation was increased to produce a test EPSP near maximal (1.5xT, prior to conditioning, $n=18)$. ${ }^{*} P<0.05$ : significant change with conditioning. This is likely because the stronger test stimulation reduced the headroom for increasing EPSPs by recruiting more proprioceptive axons, and increased self-facilitation prior to conditioning, the latter during repeated testing used to obtain EPSP averages. g, Summary of cutaneous facilitation of EPSPs from (f) (evoked by DR2 at 1.1xT), but separated into trials without (as in (b), $n=31$ ) and with (as in (d), $n=11$ ) large background postsynaptic changes induced by conditioning that lasted up to and during the EPSP testing (at 60 ms post conditioning, $\mathrm{Bkg}$ ). ${ }^{*} P<0.05$ : significant change with conditioning evoked $\mathrm{PAD} .+P<0.05$ : significant reduction facilitation with increased background activity (Bkg). $\mathbf{h}$, Remote postsynaptic inhibition from conditioning. Long lasting changes in intrinsic proprieties of motoneurons (S4 and S3) following a mixed proprioceptive and cutaneous conditioning DR stimulation (on S3 or contralateral S4 DR, 2.5xT, DR1) that only produced a transient postsynaptic depolarization that ended prior to EPSP testing (as in B), including a reduction in time constant $(\tau$ ) and slight hyperpolarization of potential $\left(V_{m}\right)$, both measured at the time of EPSP testing (measured at $60 \mathrm{~ms}$ post conditioning, but in trials without EPSP testing; rats, $n=15)$. At this time, there was little change in somatic membrane resistance $(\mathrm{Rm})$ with conditioning, suggesting that conditioning induced postsynaptic activity at a remote location in distal dendrites of the motoneuron. Indeed, when we voltage clamped the membrane potential during monosynaptic testing (DR2 at 1.1-1.5xT) to directly measure the synaptic current (EPSC) and minimize that inhibitory action of postsynaptic conductance increases, we found that the conditioning stimulation (DR1) produced a larger facilitation of the monosynaptic EPSC than the EPSP measured in current clamp in the same motoneurons. These results are consistent with the facilitation of the EPSP being masked by postsynaptic inhibition from increases in remote dendritic postsynaptic conductances triggered by the conditioning stimulation. $* P<0.05$ : significant change with conditioning. 


\section{Supplementary Table 1. Chronological list of evidence contradicting the classical concept of presynaptic inhibition of transmitter release from proprioceptive sensory axon terminals on motoneurons.}

\begin{tabular}{|c|c|c|}
\hline Date & $\begin{array}{l}\text { Contradictions in classic view of terminal presynaptic inhibition } \\
\text { mediated by terminal GABA } A \text { receptors and PAD }\end{array}$ & Resolution of contradictions \\
\hline 1938 & $\begin{array}{l}\text { Primary afferent depolarization (PAD) directly evokes spikes in sensory axons, } \\
\text { producing excitation rather than presynaptic inhibition. Barron and Matthews } \\
(1938)^{23} \text { discovered that sensory nerve conditioning evokes a long depolarization } \\
\text { in many other sensory afferents (primary afferent depolarization, PAD), which we } \\
\text { now know is mostly GABA } \text { mediated }^{113} \text {. They and others noted that sometimes } \\
\text { this PAD was large enough to directly induce axon spiking, even in vivo }{ }^{46} \text {, including } \\
\text { spikes in the sensory axons mediating the MSR itself, raising a contradiction with } \\
\text { the notion of GABA mediated presynaptic inhibition }{ }^{16} \text {. While these PAD-triggered } \\
\text { spikes do not fully propagate antidromically out the DRs in many axons (they fail } \\
\text { en route), they are actually initiated in most axons and more likely to conduct } \\
\text { orthodromically }{ }^{16} \text {, making most axons and their motoneuron synapse refractory } \\
\text { to subsequent testing (post activation depression). Indeed, numerous groups have } \\
\text { shown that these spikes directly activate the MSR pathway }{ }^{7,9,47,117} \text {. Thus, these } \\
\text { PAD-evoked spikes must inhibit afferent transmission in the MSR pathway by } \\
\text { making axons refractory and producing post activation depression of their } \\
\text { terminal synapse, even in humans where PAD evoked spikes occur }{ }^{36} \text {. This indirect } \\
\text { inhibition is GABA } \text { A }_{A} \text { mediated and thus readily mistaken for presynaptic inhibition } \\
\text { (sensitive to GABA } \text { Antagonists) }^{118,119} \text {. Even Eccles noted this issue, and showed } \\
\text { that just the refractory period alone in the sensory axon inhibits the MSR }{ }^{9} \text {. }\end{array}$ & $\begin{array}{l}\text { Post activation depression from PAD } \\
\text { evoked spikes inhibits the MSR and } \\
\text { masks facilitation of the MSR by nodal } \\
\text { facilitation. We find that facilitation of } \\
\text { the MSR by conditioning evoked PAD } \\
\text { is always reduced when it is associated } \\
\text { with a large enough conditioning } \\
\text { stimulation to evoke spikes in sensory } \\
\text { afferents, which likely results from } \\
\text { post activation depression of axon } \\
\text { transmission. This likely explains why } \\
\text { Fink et al. }{ }^{7,17} \text { recently saw inhibition of } \\
\text { the MSR with optogenetic or sensory } \\
\text { activation of GABA axo neurons. } \\
\text { Crucially, when looking for MSR } \\
\text { facilitation, avoiding these spikes and } \\
\text { post activation depression requires } \\
\text { using weak conditioning stimuli, unlike } \\
\text { previous studies }\end{array}$ \\
\hline 1949 & $\begin{array}{l}\text { Post-tetanic potentiation (PTP) of the MSR increases sensory nerve conduction, but } \\
\text { its mechanisms have remained elusive. Lloyd (1949) })^{120} \text { concluded that increasing } \\
\text { conduction along sensory axons (not just terminals) contributed to the minutes of } \\
\text { facilitation of the MSR seen after a high frequency nerve stimulation train (PTP). } \\
\text { However, he supposed this might be due to hyperpolarization of the sensory } \\
\text { axons, even though we now know that such trains depolarize axons via tonic } \\
\text { PAD }^{16} \text {. The tonic PAD from these bursts must overwhelm the hyperpolarization } \\
\text { driven by Na-K pump activity }{ }^{121} \text {. Lloyd also concluded that PTP only occurred } \\
\text { when the same nerve is used for the train (tetanus) as for testing the MSR. }\end{array}$ & $\begin{array}{l}\text { Repetitive nerve stimulation produces } \\
\text { a tonic } G A B A_{A} \text { mediated depolarization } \\
\text { (PAD) of axons that facilitates nodal } \\
\text { conduction, and increases the MSR. } \\
\text { This PAD likely contributes to PTP, and } \\
\text { is largest when the same nerve is } \\
\text { tetanized, compared to other nerves, } \\
\text { explaining why Lloyd missed the } \\
\text { subtler facilitation from other nerves. }\end{array}$ \\
\hline 1958 & $\begin{array}{l}\text { PAD is associated with a lowering of the threshold for activating spikes. Early on } \\
\text { Wall (1958) })^{31} \text { noted that a conditioning nerve stimulation that depolarized sensory } \\
\text { axons (PAD) was associated with a lower threshold to extracellularly activate } \\
\text { these axons. Subsequently this was assumed to be due to the action of terminal } \\
\text { GABA }_{A} \text { receptors and presynaptic inhibition, and spike threshold changes were } \\
\text { used to estimate the size of PAD }{ }^{113,122} \text {. }\end{array}$ & $\begin{array}{l}\text { PAD lowers the spike threshold via } \\
\text { nodal GABA } \text { receptors assisting the } \\
\text { sodium spike. This is not related to } \\
\text { presynaptic inhibition, but can still be } \\
\text { used to estimate PAD as Rudomin and } \\
\text { others have done. }\end{array}$ \\
\hline $\begin{array}{l}1957 \\
- \\
1994\end{array}$ & $\begin{array}{l}\text { PAD is not correlated with inhibition of the monosynaptic reflex (MSR). Shortly } \\
\text { after Frank and Fortes discovered that the leg extensor muscle MSR is inhibited by } \\
\text { a conditioning of a flexor nerve in cats (PBST; like Fig. } 6)^{123,124} \text {, Eccles proposed the } \\
\text { concept of presynaptic inhibition mediated by this conditioning depolarizing of the } \\
\text { proprioceptive sensory axon terminals in the MSR pathway (PAD), simply because } \\
\text { the MSR and PAD are somewhat correlated in time } 9 \text {. However, in retrospect PAD } \\
\text { is far too brief to account for the much longer inhibition caused by this } \\
\text { conditioning } 43,115 \text {, and some flexor nerve conditioning (a single PBST pulse) } \\
\text { inhibits the MSR (Fig. } 1 \text { of Eccles, } 1961^{9} \text { ), even though it does not cause PAD in the } \\
\text { extensor proprioceptors of the MSR at all }{ }^{125} \text {. }\end{array}$ & $\begin{array}{l}\text { PAD is correlated with nodal spike } \\
\text { facilitation and facilitation of the MSR. } \\
\text { PAD causes facilitation of the MSR, } \\
\text { explaining this correlation. When PAD } \\
\text { is large and evokes axonal spikes, } \\
\text { these cause post activation depression } \\
\text { (detailed above) that should also be } \\
\text { correlated with PAD, but is not due to } \\
\text { presynaptic inhibition. Also, } \\
\text { barbiturates used by Eccles and others } \\
\text { potentiated GABA } \text { receptor currents. }\end{array}$ \\
\hline $\begin{array}{l}1959 \\
- \\
1993\end{array}$ & $\begin{array}{l}\text { Postsynaptic inhibition inevitably accounts for part of the inhibition of the MSR by } \\
\text { flexor nerve conditioning. In his initial short report Frank }(1959)^{123} \text { correctly } \\
\text { suggested that the early inhibition of the MSR by flexor nerve conditioning might } \\
\text { be partly postsynaptic (rather than presynaptic), on motoneuron distal dendrites. } \\
\text { Others dismissed postsynaptic inhibition because the decay times of the EPSP } \\
\text { does not always change when the EPSP is reduced by conditioning, which they } \\
\text { proposed indicated that there was no postsynaptic change in conductance in } \\
\text { distal dendrites }{ }^{45,113} \text {. However, this method is likely not very sensitive (due to } \\
\text { variability in unitary EPSP time course). Also, anatomically } ~ 70 \% \text { of GABA axo }\end{array}$ & $\begin{array}{l}\text { Postsynaptic inhibition masks } \\
\text { facilitation of the MSR by nodal } \\
\text { facilitation. We find evidence for long } \\
\text { lasting postsynaptic inhibition on distal } \\
\text { motoneuron dendrites during nerve } \\
\text { conditioning stimulation (including } \\
\text { postsynaptic reductions in Tau, Vm } \\
\text { and unitary EPSP heights and single } \\
\text { MU firing). Crucially, minimizing }\end{array}$ \\
\hline
\end{tabular}




\begin{tabular}{|c|c|c|}
\hline & $\begin{array}{l}\text { contacts on afferent terminals also contact motoneurons (in a triad), so } \\
\text { postsynaptic inhibition is inevitable }{ }^{8,44} \text {. }\end{array}$ & $\begin{array}{l}\text { postsynaptic inhibition requires using } \\
\text { a small conditioning stimulation when } \\
\text { looking for MSR facilitation, unlike } \\
\text { previous studies }{ }^{18} \text {. }\end{array}$ \\
\hline $\begin{array}{l}1961 \\
- \\
2014\end{array}$ & $\begin{array}{l}\text { Self-facilitation during repeated MSR testing reduces the possibility of observing } \\
\text { facilitation with subsequent conditioning stimuli, leaving only inhibitory actions of } \\
\text { conditioning. Eccles and others knew that the same proprioceptive nerve } \\
\text { stimulation that activates the MSR also depolarizes these proprioceptive afferents } \\
\text { (PAD self-activation) }{ }^{9} \text {. Thus, just the act of repeatedly testing the MSR to find the } \\
\text { average MSR prior to conditioning pre-activates PAD and produces self-facilitation } \\
\text { of the MSR, reducing the headroom to observe changes in the MSR following a } \\
\text { separate nerve conditioning stimulation that produces PAD. However, at the time } \\
\text { it was not known that repeated nerve stimulation causes a tonic buildup of GABA } \\
\text { and a tonic PAD that alters sensory transmission and MSR even at long repetition } \\
\text { intervals of many seconds. Thus, Eccles and others used short test intervals (1 s) } \\
\text { and strong maximal MSR test stimuli 7,9,18, presumably assuming that there would } \\
\text { be no interaction between test stimuli, which is not the case. In retrospect, these } \\
\text { short test intervals and strong test stimuli must have preactivated tonic GABA, } \\
\text { leaving little headroom to observe facilitation of the MSR (facilitation), and leaving } \\
\text { mainly only inhibitory action possible. }\end{array}$ & $\begin{array}{l}\text { Self-facilitation masks facilitation of } \\
\text { the MSR by a conditioning stimulation. } \\
\text { To observe facilitation of the MSR by a } \\
\text { conditioning stimuli that produces a } \\
\text { PAD it is important to use long test } \\
\text { intervals }(5-10 \text { s) and small MSR test } \\
\text { intensities }(1.1 \times T) \text { to minimize self } \\
\text { activation of a tonic PAD prior to } \\
\text { conditioning. While experimentally } \\
\text { troublesome, self facilitation during } \\
\text { repetitive activation is actually one of } \\
\text { the main functions of PAD, allowing } \\
\text { sensory axons to faithfully transmit } \\
\text { spikes to motoneurons at high } \\
\text { frequencies that would otherwise } \\
\text { produce sodium spike inactivation. }\end{array}$ \\
\hline 1980 & $\begin{array}{l}\text { Sensory axon terminal potentials at motoneurons are consistent with spike failure. } \\
\text { Early efforts to examine how spikes propagated to sensory axon terminals } \\
\text { employed extracellular recordings (EC) near the motoneurons, called terminal } \\
\text { potentials (Sypert et al. 1980) }{ }^{116} \text {. However, unlike EC recordings from near } \\
\text { conducting axons (Fig. } 2 \text { b), these terminal potentials lacked much of the obvious } \\
\text { negative field associated with the action potential, and instead had a prominent } \\
\text { positive field, followed by a smaller negative field (Extended Data Fig. } 9 \text { and } \\
\text { Sypert }{ }^{116} \text { ). This positive field has been shown in other axons to be indicative of } \\
\text { spike propagation failure and result from the passive axonal current caused by the } \\
\text { last non-failing node, similar to a FP, as demonstrated in motor axon recordings } \\
71,73 \text {. Indeed, we found that even dorsal horn recordings could exhibit this positive } \\
\text { field if the nearby dorsal root conduction is blocked with a microinjection of TTX } \\
\text { (Extended Data Fig. } 9 \mathrm{~d} \text { ). Sypert }{ }^{116} \text { went on to show that with PAD evoked by nerve } \\
\text { conditioning this positive terminal potential field was decreased, and incorrectly } \\
\text { interpreted this as evidence for decreased spike conduction and thus supposed it } \\
\text { was due to presynaptic inhibition. }\end{array}$ & $\begin{array}{l}\text { Positive terminal potential fields are } \\
\text { decreased with PAD, indicative of } \\
\text { decreased conduction failure, and in } \\
\text { retrospect consistent with Sypert }{ }^{116} \text {. } \\
\text { There is a small negative field that } \\
\text { follows the positive field in terminal } \\
\text { potential recordings, representing } \\
\text { spikes that actually reach the } \\
\text { terminals. We quantified negative field } \\
\text { and found it to increase with PAD, } \\
\text { consistent again with increased spikes } \\
\text { conducting to motoneurons (Extended } \\
\text { Data Fig. 9). Blocking activity in the } \\
\text { spinal cord with glutamate } \\
\text { antagonists, which would include } \\
\text { blocking GABA } \text { axo }_{\text {arcuit activity, }} \\
\text { decreased this negative field. }\end{array}$ \\
\hline & 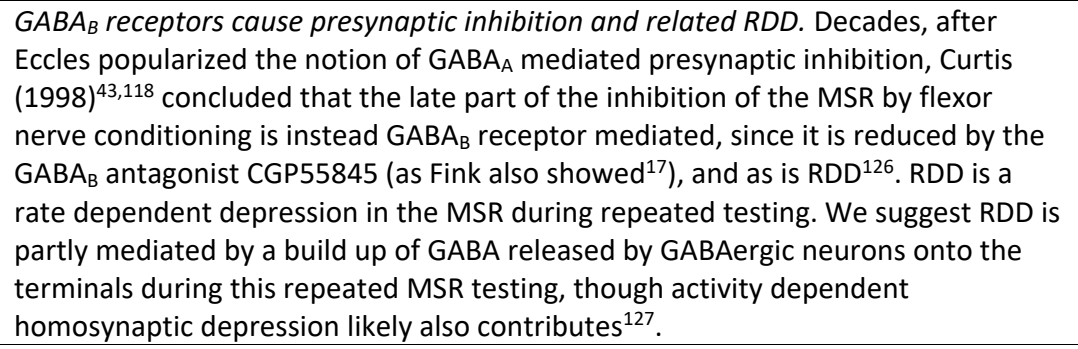 & $\begin{array}{l}\text { GABA } A_{B} \text { mediated presynaptic inhibition } \\
\text { masks facilitation of the MSR by nodal } \text { GABA }_{A} \text { receptors. GABA } \\
\text { loceceptors are } \\
\text { loced on the terminals, and produce } \\
\text { presynaptic inhibition (Fig. 5) and RDD } \\
\text { (Bennett and Hari, unpublished } \\
\text { results), which are reduced by GABA } \\
\text { antagonists (CGP55845) or silencing } \\
\text { GABA }_{\text {axo }} \text { neurons. }\end{array}$ \\
\hline $\begin{array}{l}1990 \\
- \\
1998\end{array}$ & $\begin{array}{l}G A B A_{A} \text { receptors have direct postsynaptic inhibitory effects on many spinal } \\
\text { neurons, making the actions of } G A B A_{A} \text { antagonists difficult to attribute to } \\
\text { presynaptic inhibition. By the } 1990 \text { s Redman and others tried to confirm the role } \\
\text { of GABA } A_{A} \text { receptors in presynaptic inhibition by locally applying the GABA } A \\
\text { antagonists bicuculline to the spinal cord, and indeed found this drug or other } \\
\text { antagonists reduced the inhibition of the MSR by flexor nerve conditioning } \\
18,43,118,128 \text {. However, we now know that this is indirectly due to bicuculline causing } \\
\text { a widespread disinhibition of the spinal cord (including loss of post activation } \\
\text { depression, detailed above) that leads to a convulsive spinal cord with very long } \\
\text { lasting polysynaptic reflexes evoked by the nerve conditioning or the MSR testing } \\
\text { itself, making pre and postsynaptic actions hard to distinguish. Further, we know } \\
\text { that GABA } A_{A} \text { receptors mediate dorsal root reflexes and associated post activation } \\
\text { depression of the MSR (see above point), and thus bicuculline and picrotoxin likely } \\
\text { reduce the inhibition of the MSR via reducing post activation depression (see } \\
\text { above), rather than changing presynaptic inhibition. }\end{array}$ & $\begin{array}{l}G A B A_{A} \text { receptor antagonists reduce the } \\
M S R \text {, by reducing nodal facilitation. } \\
\text { Postsynaptic } \mathrm{GABA}_{\mathrm{A}} \text { receptors have } \\
\text { potent inhibitory actions on many } \\
\text { spinal neurons involved in } \\
\text { polysynaptic reflexes. However, } \\
\text { minimizing these polysynaptic reflexes } \\
\text { (by using weak test stimuli and } \\
\text { blocking NMDA receptors) reveals a } \\
\text { direct inhibition of the MSR by GABA } \\
\text { antagonists, as does optogenetically } \\
\text { silencing } \mathrm{GABA}_{\text {axo }} \text { neuron, consistent } \\
\text { with GABA } \mathrm{A}_{\mathrm{A}} \text { receptors facilitating } \\
\text { rather than inhibiting sensory } \\
\text { transmission. }\end{array}$ \\
\hline
\end{tabular}




\begin{tabular}{|c|c|c|}
\hline $\begin{array}{l}1990 \\
- \\
1995\end{array}$ & $\begin{array}{l}\text { PAD recorded in the dorsal roots cannot arise from terminal GABA receptors, due } \\
\text { to spatial attenuation on the axon. With advent of detailed anatomical and } \\
\text { computer models of sensory axons }{ }^{34,129,130} \text { it became clear that signals like spikes } \\
\text { or PAD are attenuated over short distances in axons, }<200 \mu \mathrm{m} \text {. This implies that } \\
\text { PAD recorded on or near the DR is unlikely to bare any relation to terminal } \\
\text { presynaptic inhibition, despite claims to the contrary } 7,37,113,128 \text {. }\end{array}$ & $\begin{array}{l}\text { Space constant } \lambda_{S} \text { of sensory axons is } \\
\text { about } 90 \mu \mathrm{m} \text {. Thus, the PAD recorded } \\
\text { in the dorsal root must arise from } \\
\text { nearby nodal GABA receptors, and not } \\
\text { bear any relation to GABA action at } \\
\text { the terminals }>1000 \mu \mathrm{m} \text { away. }\end{array}$ \\
\hline $\begin{array}{l}1994 \\
- \\
1999\end{array}$ & $\begin{array}{l}\text { Shunting inhibition produced by axon terminal GABA } A_{A} \text { receptors is not adequate to } \\
\text { produce presynaptic inhibition of the } M S R \text {. Numerous invertebrate studies } \\
\text { proposed that the conductance from } \mathrm{GABA}_{\mathrm{A}} \text { receptors in terminals caused a } \\
\text { reduction in spike height via its shunting action that contributed to presynaptic } \\
\text { inhibition with nerve conditioning }{ }^{24} \text {. However, the effects of conditioning on } \\
\text { spikes was small and terminals were not actually recorded from. Subsequently } \\
\text { modelling considerations led to the conclusion that shunting inhibition is not } \\
\text { adequate to produce presynaptic inhibition and calcium was somehow } \\
\text { involved }{ }^{129}, \text { possibly further implicating GABA } \mathrm{A}_{B} \text { receptors, as we see. Considering } \\
\text { our estimated space constant } \lambda_{S} \text { of } \sim 90 \mu \mathrm{m} \text {, the small shunting inhibition of the } \\
\text { spike height ( } 1 \mathrm{mV} \text { ) we observe is very unlikely to prevent the spike produced at a } \\
\text { given node from activating a downstream neighbouring node, since nodes are } \sim 50 \\
\mu m \text { apart, leading to only about a } 50 \% \text { reduction in spike height at the } \\
\text { downstream node (to } \sim 40 \text { mV, unless of course the node is failing), which is well } \\
\text { above that needed to initiate a full nodal spike. Thus, spike propagation is very } \\
\text { unlikely to be blocked by shunting inhibition. Also, terminal boutons are mostly on } \\
\text { unmyelinated axons without sodium channels (passive, 3rd order), and so a } 1 \% \\
\text { reduction in the spike arising from the last/closest node on the } 2 \text { nd order branch } \\
\text { will have little effect on the terminal depolarization (1\%), ruling out substantial } \\
\text { shunting inhibition of transmitter release from the terminal. }\end{array}$ & $\begin{array}{l}\text { GABA }_{A} \text { receptors only slightly decrease } \\
\text { spikes by shunting conductances, and } \\
\text { otherwise assist nodal spike } \\
\text { conduction in proprioceptive axons. In } \\
\text { non-failing secure spikes in sensory } \\
\text { axons GABA receptors lower the } \\
\text { threshold for spike activation } \\
\text { (rheobase) and speed the spikes, the } \\
\text { latter by decreasing the time constant } \\
\text { of the axon (RC). They do decrease the } \\
\text { spike, but only by about } 1 \% \text {, consistent } \\
\text { with shunting being unlikely to inhibit } \\
\text { spike transmission to motoneurons. } \\
\text { However, this does not rule out } \\
\text { densely expressed GABA } \text { receptors } \\
\text { causing shunting and presynaptic } \\
\text { inhibition in cutaneous afferents, as } \\
\text { previously suggested }{ }^{16,70,131} \text {. }\end{array}$ \\
\hline $\begin{array}{l}1994 \\
- \\
1998\end{array}$ & $\begin{array}{l}\text { Sodium spike inactivation from axon terminal } G A B A_{A} \text { receptor depolarization is not } \\
\text { adequate to produce presynaptic inhibition. Early poor quality recordings from } \\
\text { sensory axons (resting near }-50 \mathrm{mV} \text { from penetration injury) } \\
\text { prevailing view that spike failure with depolarization (PAD) was much more } \\
\text { common than we now find with better recordings (resting near }-70 \mathrm{mV} \text {, Extended } \\
\text { Data Fig. 3b). Further, Redman later questioned this view, and it seems unlikely } \\
\text { for the MSR pathway }{ }^{18,119} \text {. }\end{array}$ & $\begin{array}{l}\text { Physiological PAD depolarizations do } \\
\text { not block proprioceptive sensory axons } \\
\text { spikes, and instead prevent them from } \\
\text { failing in the MSR pathway. However, } \\
\text { this does not rule out densely } \\
\text { expressed } \mathrm{GABA}_{\mathrm{A}} \text { receptors causing } \\
\text { spike inactivation in other axons. }\end{array}$ \\
\hline $\begin{array}{l}1995 \\
- \\
1998\end{array}$ & 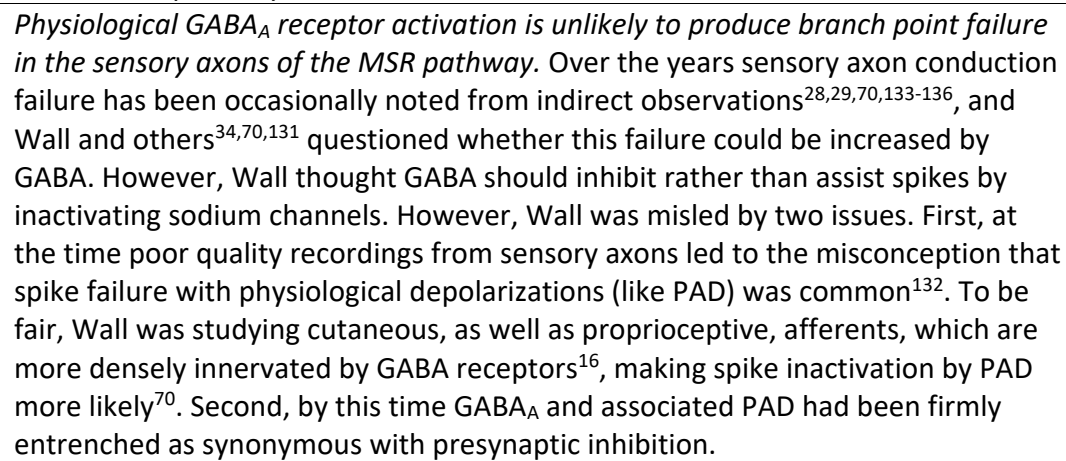 & $\begin{array}{l}\text { GABA }_{A} \text { receptors help prevent branch } \\
\text { point failure and thus facilitate sensory } \\
\text { transmission in the MSR. Computer } \\
\text { simulations by Walmsley and others } \\
34,129 \text { have led to the conclusion that } \\
\text { physiological GABA }{ }_{A} \text { receptor } \\
\text { conductances cannot stop spikes from } \\
\text { propagating past a node. Instead we } \\
\text { report here that they help prevent } \\
\text { spike failure at branch points, } \\
\text { including in our computer simulations. }\end{array}$ \\
\hline $\begin{array}{l}1996 \\
- \\
2018\end{array}$ & $\begin{array}{l}\text { Little or no terminal } G A B A_{A} \text { receptors on proprioceptive axons. Extrasynaptic } \alpha 5 \\
\mathrm{GABA}_{A} \text { receptors were found to be lacking at most proprioceptive axon terminals } \\
\text { in the ventral horn }{ }^{16} \text {. Synaptic } G A B A_{A} \text { receptor labelling on these terminals had } \\
\text { not been directly addressed, though see } 6,7,137 . \mathrm{GABA}_{B} \text { receptor immunolabelling } \\
\text { had also not been investigated in these afferents, though is has in others }(A \beta)^{138} \text {. }\end{array}$ & $\begin{array}{l}G A B A_{A} \text { receptors are mostly at nodes, } \\
\text { whereas } G A B A_{B} \text { receptors are at } \\
\text { terminals in large proprioceptive } \\
\text { afferents. }\end{array}$ \\
\hline $\begin{array}{l}2005 \\
- \\
2014\end{array}$ & $\begin{array}{l}\text { When GAD2 expressing GABAergic neurons were identified that make axoaxonic } \\
\text { connections onto terminals of proprioceptive sensory axons }{ }^{6-8} \text { they were presumed } \\
\text { to mainly produce presynaptic inhibition. Thus, they were called GABA pre neurons } \\
\text { (also called P-bouton neurons), unfortunately implying that their only role is only } \\
\text { presynaptic inhibition. We have instead referred to these neurons by the more } \\
\text { generic termed GABA }{ }_{a x o} \text {. }\end{array}$ & $\begin{array}{l}\text { A key role of } \mathrm{GABA}_{\mathrm{axo}} \text { neurons is to } \\
\text { innervate proprioceptive afferent } \\
\text { nodes via } \mathrm{GABA}_{A} \text { receptors and ventral } \\
\text { terminals via } \mathrm{GABA}_{B} \text { receptors, } \\
\text { producing nodal facilitation and } \\
\text { presynaptic inhibition, respectively. }\end{array}$ \\
\hline 2018 & $\begin{array}{l}\text { GABA } A_{a x o} \text { neuron activation by sensory conditioning does not depolarize } \\
\text { proprioceptive axon terminals. Direct recordings from the fine terminals of } \\
\text { proprioceptive afferents reveal that during sensory conditioning the terminal is } \\
\text { not depolarized during the long PAD recorded on dorsal roots }{ }^{16} \text {. }\end{array}$ & $\begin{array}{l}\text { GABA axo neuron activation depolarizes } \\
\text { nodes. Dorsally located nodes produce } \\
\text { the PAD recorded dorsal roots. }\end{array}$ \\
\hline
\end{tabular}




\section{Supplementary Table 2. Resources used in Methods.}

\begin{tabular}{|c|c|c|}
\hline REAGENT or RESOURCE & SOURCE & IDENTIFIER \\
\hline \multicolumn{3}{|l|}{ Antibodies } \\
\hline Rabbit anti- $\alpha_{5} G_{A B A}$ receptor subunit & OriGene Tech. & TA338505 \\
\hline Rabbit anti- $\alpha_{1} \mathrm{GABA}_{\mathrm{A}}$ receptor subunit & Sigma-Aldrich & $06-868$ \\
\hline Guinea pig anti- $\alpha_{2} \mathrm{GABA}_{\mathrm{A}}$ receptor subunit & Synaptic Systems & 224104 \\
\hline Chicken anti- $\gamma_{2} \mathrm{GABA}_{\mathrm{A}}$ receptor subunit & Synaptic Systems & 224006 \\
\hline Rabbit anti-GABA $A_{B 1}$ receptor subunit & Synaptic Systems & 322102 \\
\hline Mouse anti-NF200 (Neurofilament 200) & Sigma-Aldrich & N0142 \\
\hline Guinea pig anti-VGLUT1 (Vesicular glutamate transporter 1) & Sigma-Aldrich & AB5905 \\
\hline Rabbit anti-Caspr (Contactin associated protein) & Abcam & ab34151 \\
\hline Mouse anti-Caspr (Contactin associated protein) & NeuroMab & K65/35 \\
\hline Chicken anti-MBP (Myelin basic protein) & Abcam & ab106583 \\
\hline $\begin{array}{l}\text { Chicken anti-VGAT (Vesicular inhibitory amino acid } \\
\text { transporter) }\end{array}$ & Synaptic Systems & 131006 \\
\hline Rabbit anti-VGAT & Sigma-Aldrich & AB5062P \\
\hline Rabbit anti-EYFP (Enhanced yellow fluorescent protein) & Biorbyt & orb256069 \\
\hline Goat anti-RFP (Red fluorescent protein; binds tdTom) & Biorbyt & orb334992 \\
\hline Rabbit anti-RFP (Red fluorescent protein; binds tdTom) & MBL Int. & PM005 \\
\hline Rabbit anti-GFP (Green fluorescent protein) & ThermoFisher Sc. & A11122 \\
\hline Mouse anti-Pan Sodium Channel (binds all Nav types) & Sigma-Aldrich & S8809 \\
\hline Goat anti-rabbit Alexa Fluor 555 & ThermoFisher Sc. & A32732 \\
\hline Goat anti-rabbit Alexa Fluor 647 & Abcam & ab150079 \\
\hline Goat anti-rabbit Pacific orange & ThermoFisher Sc. & P31584 \\
\hline Goat anti-mouse Alexa Fluor 647 & ThermoFisher Sc. & A21235 \\
\hline Goat anti-mouse Alexa Fluor 488 & ThermoFisher Sc. & A11001 \\
\hline Goat anti-mouse Alexa Fluor 555 & ThermoFisher Sc. & A28180 \\
\hline Goat anti-guinea pig Alexa Fluor 647 & ThermoFisher Sc. & A21450 \\
\hline Goat anti-chicken Alexa Fluor 405 & Abcam & ab175674 \\
\hline Donkey anti-goat Alexa Fluor 555 & Abcam & ab150130 \\
\hline Donkey anti-rabbit Alexa Fluor 488 & ThermoFisher Sc. & A21206 \\
\hline Streptavidin-conjugated Alexa Fluor 488 & Jackson immunoR. & $016-540-084$ \\
\hline Streptavidin-conjugated Cyanine Cy5 & Jackson immunoR. & 016-170-084 \\
\hline \multicolumn{3}{|l|}{ Chemicals, Peptides, and Recombinant Proteins } \\
\hline M.O.M (Mouse on Mouse Immunodetection Kit) & Vector & BMK-2202 \\
\hline \multicolumn{3}{|l|}{ Experimental Models: Organisms/Strains } \\
\hline $\begin{array}{l}\text { Gad2 } 2^{\text {CreER }} \text { mouse: } \\
\text { Gad2 }^{\text {tm1(cre/ERT2)Zjh } / \mathrm{J}}\end{array}$ & The Jackson Laboratory & Stock\# 010702 \\
\hline $\begin{array}{l}\text { Vglut1 }^{\text {cre }} \text { mouse: } \\
\text { B6;129S-SIc17a7tm1.1(cre)Hze } / \mathrm{J}\end{array}$ & The Jackson Laboratory & Stock\# 023527 \\
\hline 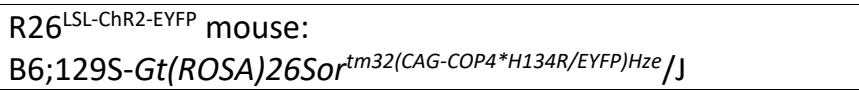 & The Jackson Laboratory & Stock\# 012569 \\
\hline 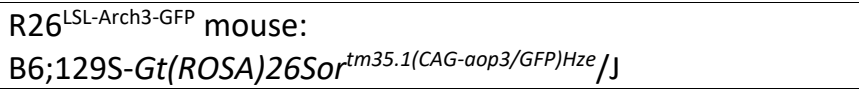 & The Jackson Laboratory & Stock\# 012735 \\
\hline $\begin{array}{l}\text { R26 }{ }^{\text {LSL-tdTom }} \text { mouse crossed with Gad2 }{ }^{\text {CreER }} \text { mice: } \\
\text { B6.Cg-Gt(ROSA)26Sortm14(CAG-tdTomato)Hze } / \mathrm{J}\end{array}$ & The Jackson Laboratory & Stock\# 007914 \\
\hline 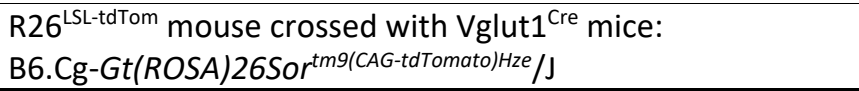 & The Jackson Laboratory & Stock\# 007909 \\
\hline
\end{tabular}




\begin{tabular}{|c|c|c|}
\hline \multicolumn{3}{|l|}{ Oligonucleotides } \\
\hline $\begin{array}{l}\text { 5' -> ACG TTT CCT GTC CCT GTG TG -> 3' } \\
\text { Common for Gad2 }{ }^{\text {CreER }} \text { mice }\end{array}$ & $\begin{array}{l}\text { Integrated DNA } \\
\text { technologies }\end{array}$ & 11400 \\
\hline $\begin{array}{l}5^{\prime} \text {-> AGG CAA ATT TTG GTG TAC GG -> } 3^{\prime} \\
\text { Mutant for Gad2 } 2^{\text {Creer }} \text { mice }\end{array}$ & $\begin{array}{l}\text { Integrated DNA } \\
\text { technologies }\end{array}$ & olMR9074 \\
\hline $\begin{array}{l}5^{\prime} \text {-> CAG ACG CTG CAG TCT TTC AG -> 3' } \\
\text { Wild type for Gad2 }{ }^{\text {Creer }} \text { mice }\end{array}$ & $\begin{array}{l}\text { Integrated DNA } \\
\text { technologies }\end{array}$ & olMR3346 \\
\hline $\begin{array}{l}5^{\prime} \text {-> ACA TGG TCC TGC TGG AGT TC -> 3' } \\
\text { Mutant Forward for ChR2 mice }\end{array}$ & $\begin{array}{l}\text { Integrated DNA } \\
\text { technologies }\end{array}$ & olMR9102 \\
\hline $\begin{array}{l}\text { 5' -> GGC ATT AAA GCA GCG TAT CC }->3^{\prime} \\
\text { Mutant Reverse for ChR2 mice }\end{array}$ & $\begin{array}{l}\text { Integrated DNA } \\
\text { technologies }\end{array}$ & olMR9103 \\
\hline $\begin{array}{l}5^{\prime} \text {-> AAG GGA GCT GCA GTG GAG TA -> 3' } \\
\text { Wild type Forward for ChR2 mice }\end{array}$ & $\begin{array}{l}\text { Integrated DNA } \\
\text { technologies }\end{array}$ & olMR9020 \\
\hline $\begin{array}{l}5^{\prime} \text {-> CCG AAA ATC TGT GGG AAG TC -> 3' } \\
\text { Wild type Reverse for ChR2 mice }\end{array}$ & $\begin{array}{l}\text { Integrated DNA } \\
\text { technologies }\end{array}$ & olMR9021 \\
\hline $\begin{array}{l}5^{\prime} \text {-> CTG TTC CTG TAC GGC ATG G -> } 3^{\prime} \\
\text { Mutant Forward for both tdTom mouse strains }\end{array}$ & $\begin{array}{l}\text { Integrated DNA } \\
\text { technologies }\end{array}$ & olMR9105 \\
\hline $\begin{array}{l}5^{\prime} \text {-> GGC ATT AAA GCA GCG TAT CC }->3^{\prime} \\
\text { Mutant Reverse for both tdTom mouse strains }\end{array}$ & $\begin{array}{l}\text { Integrated DNA } \\
\text { technologies }\end{array}$ & olMR9103 \\
\hline $\begin{array}{l}5^{\prime} \text {-> AAG GGA GCT GCA GTG GAG TA -> 3' } \\
\text { Wild type Forward for both tdTom mouse strains }\end{array}$ & $\begin{array}{l}\text { Integrated DNA } \\
\text { technologies }\end{array}$ & olMR9020 \\
\hline $\begin{array}{l}5^{\prime}->\text { CCG AAA ATC TGT GGG AAG TC }->3^{\prime} \\
\text { Wild type Reverse for both tdTom mouse strains }\end{array}$ & $\begin{array}{l}\text { Integrated DNA } \\
\text { technologies }\end{array}$ & olMR9021 \\
\hline $\begin{array}{l}5^{\prime} \text {-> CTT CTC GCT AAG GTG GAT CG -> 3' } \\
\text { Mutant Forward for Arch3 mice }\end{array}$ & $\begin{array}{l}\text { Integrated DNA } \\
\text { technologies }\end{array}$ & 12178 \\
\hline $\begin{array}{l}5^{\prime} \text {-> CAC CAA GAC CAG AGC TGT CA }>>3 \\
\text { Mutant Reverse for Arch3 mice }\end{array}$ & $\begin{array}{l}\text { Integrated DNA } \\
\text { technologies }\end{array}$ & 12179 \\
\hline $\begin{array}{l}5^{\prime} \text {-> TCC CAA AGT CGC TCT GAG TT -> 3' } \\
\text { Wild type Forward for Arch3 mice }\end{array}$ & $\begin{array}{l}\text { Integrated DNA } \\
\text { technologies }\end{array}$ & olMR8713 \\
\hline $\begin{array}{l}5^{\prime}->\text { CTT TAA GCC TGC CCA GAA GA -> 3' } \\
\text { Wild type Reverse for Arch3 mice }\end{array}$ & $\begin{array}{l}\text { Integrated DNA } \\
\text { technologies }\end{array}$ & 12177 \\
\hline $\begin{array}{l}5^{\prime} \text {-> ATG AGC GAG GAG AAG TGT GG -> 3' } \\
\text { Common for VGLUT1 }{ }^{\prime} \text { re mice }\end{array}$ & $\begin{array}{l}\text { Integrated DNA } \\
\text { technologies }\end{array}$ & 17904 \\
\hline $\begin{array}{l}5^{\prime}->\text { CCC TAG GAA TGC TCG TCA AG -> } 3^{\prime} \\
\text { Mutant reverse for VGLUT1 }{ }^{\text {cre }} \text { mice }\end{array}$ & $\begin{array}{l}\text { Integrated DNA } \\
\text { technologies }\end{array}$ & 12231 \\
\hline $\begin{array}{l}5^{\prime} \text {-> GTG GAA GTC CTG GAA ACT GC -> 3' } \\
\text { Wild type reverse for VGLUT1 } 1^{\text {cre }} \text { mice }\end{array}$ & $\begin{array}{l}\text { Integrated DNA } \\
\text { technologies }\end{array}$ & 17905 \\
\hline
\end{tabular}


bioRxiv preprint doi: https://doi.org/10.1101/2021.01.20.427494; this version posted March 28, 2021. The copyright holder for this preprint (which was not certified by peer review) is the author/funder. All rights reserved. No reuse allowed without permission.

\begin{tabular}{|l|l|l|}
\hline & & \\
\hline Software and Algorithms & & \\
\hline Leica Application Suite X software & Leica Microsystems & \\
\hline Clampfit 8.0 & Axon Instruments & \\
\hline & & \\
\hline
\end{tabular}

